JOURNAL OF THE

AMERICAN MATHEMATICAL SOCIETY

Volume 18, Number 4, Pages 823-872

S 0894-0347(05)00490-X

Article electronically published on May 25, 2005

\title{
THE COHOMOLOGICAL EQUATION FOR ROTH-TYPE INTERVAL EXCHANGE MAPS
}

\author{
S. MARMI, P. MOUSSA, AND J.-C. YOCCOZ
}

\section{Contents}

0. Introduction

0.1. Interval exchange maps

0.2. The cohomological equation

0.3. Summary of the contents

1. The continued fraction algorithm for interval exchange maps

1.1. Interval exchange maps

1.2. The continued fraction algorithm

1.3. Roth-type interval exchange maps

2. The cohomological equation

2.1. The theorem of Gottschalk and Hedlund

2.2. Special Birkhoff sums

2.3. Estimates for functions of bounded variation

2.4. Primitives of functions of bounded variation

3. Suspensions of interval exchange maps

3.1. Suspension data

3.2. Construction of a Riemann surface

3.3. Compactification of $M_{\zeta}^{*}$

3.4. The cohomological equation for higher smoothness

4. Proof of full measure for Roth type

4.1. The basic operation of the algorithm for suspensions

4.2. The Teichmüller flow

4.3. The absolutely continuous invariant measure

4.4. Integrability of $\log \left\|Z_{(1)}\right\|$

4.5. Conditions (b) and (c) have full measure

4.6. The main step

4.7. Condition (a) has full measure

4.8. Proof of the proposition

Appendix A. Roth-type conditions in a concrete family of interval exchange maps

Appendix B. A nonuniquely ergodic interval exchange map satisfying condition (a)

Received by the editors April 7, 2004.

2000 Mathematics Subject Classification. Primary 37A20; Secondary 11K50, 32G15, 37A45, $37 \mathrm{E} 05$.

(C)2005 American Mathematical Society Reverts to public domain 28 years from publication 
Acknowledgements

References

\section{INTRODUCTION}

Let $\alpha$ be an irrational number, $\left(q_{n}\right)_{n \in \mathbb{N}}$ be the sequence of the denominators of its continued fraction expansion and $\left(a_{n}\right)_{n \in \mathbb{N}}$ be the sequence of its partial quotients. Roth-type irrationals have several equivalent arithmetical characterizations:

- in terms of the rate of approximation by rational numbers: for all $\varepsilon>0$ there exists a positive constant $C_{\varepsilon}$ such that $|q \alpha-p| \geq C_{\varepsilon} q^{-(1+\varepsilon)}$ for all rationals $p / q$;

- in terms of the growth rate of the denominators of the continued fraction: $q_{n+1}=\mathrm{O}\left(q_{n}^{1+\varepsilon}\right)$ for all $\varepsilon>0$;

- in terms of the growth rate of the partial quotients: $a_{n+1}=\mathrm{O}\left(q_{n}^{\varepsilon}\right)$ for all $\varepsilon>0$.

In addition to these purely arithmetical characterizations an equivalent definition arises naturally in the study of the cohomological equation associated to the rotation $R_{\alpha}: x \mapsto x+\alpha$ on the circle $\mathbb{T}=\mathbb{R} / \mathbb{Z}: \alpha$ is of Roth type if and only if for all $r, s \in \mathbb{R}$ with $r>s+1 \geq 1$ and for all functions $\Phi$ of class $\mathcal{C}^{r}$ on $\mathbb{T}$ with zero mean $\int_{\mathbb{T}} \Phi d x=0$ there exists a unique function $\Psi$ of class $\mathcal{C}^{s}$ on $\mathbb{T}$ and with zero mean such that $\Psi-\Psi \circ R_{\alpha}=\Phi$.

The class of Roth-type irrationals enjoys several nice properties: by the celebrated theorem of Roth all algebraic irrationals are of Roth type. Moreover the set of Roth-type numbers has full measure and is invariant under the natural action of the modular group SL $(2, \mathbb{Z})$.

The goal of this paper is to characterize a class of interval exchange maps (i.e.m.'s) with similar properties (especially for the solutions of the associated cohomological equation and the fact of being a full measure class).

0.1. Interval exchange maps. Let $\mathcal{A}$ denote an alphabet with $d \geq 2$ elements. Let $I$ be an interval and $\left(I_{\alpha}\right)_{\alpha \in \mathcal{A}}$ a partition of $I$ into $d$ subintervals. An interval exchange map $T$ is an invertible map of $I$ which is a translation on each $I_{\alpha}$. Thus $T$ is orientation-preserving and preserves Lebesgue measure.

When $d=2$ then $T$ is just a rotation (modulo identification of the endpoints of $I$ ). It can be thought of as the first return map of a linear flow on a two-dimensional torus on a transversal circle. Analogously when $d \geq 3$ by singular suspension any i.e.m. is related to the linear flow on a suitable translation surface (see, e.g. V1] for details, or section 3 below) typically having genus greater than 2 . A well-known dictionary between translation surfaces and Riemann surfaces relates i.e.m.'s to the theory of measured foliations on surfaces (see, e.g. [FLP] for an introduction to measured foliations). Finally i.e.m.'s are related to the study of rational polygonal billiards (see $\mathrm{Ar}$, $\mathrm{Ta}$ and $[\mathrm{KH}$, Chapter 14, for a general introduction to i.e.m.'s, flows on surfaces and polygonal billiards).

Typical i.e.m.'s are minimal (this is guaranteed by a condition due to Keane [Ke1, which is automatically dealt with if the interval lengths are rationally independent) but note that ergodic properties of minimal i.e.m.'s can differ substantially from those of circle rotations: first they need not be uniquely ergodic $\mathrm{Ke} 2, \mathrm{KN}, \mathrm{Co}$, 
and second, being ergodic they can be weakly mixing [KS, V3, V4]. On the other hand uniquely ergodic i.e.m.'s are generic $[\mathrm{KR}]$ and Keane's conjecture that almost every i.e.m. is uniquely ergodic was proven independently by Masur and Veech Ma, V2]; see also Ker, Re.

One of the most important consequences for us of Keane's condition is that it allows us to introduce and to iterate indefinitely continued fraction algorithms that generalize the classical algorithm (corresponding to the choice $d=2$ ) [Ra, V2, Z1]. Both the Rauzy-Veech continued fraction algorithm and its accelerated version due to Zorich are ergodic w.r.t. an absolutely continous invariant measure in the space of i.e.m.'s. However in the case of the Rauzy-Veech continued fraction the measure has infinite mass whereas the invariant measure for the Zorich algorithm has finite mass. The ergodic properties of the continued fraction map and of the related Teichmüller flow (see Section 4.2 for its definition) have been studied in detail [V5, V6, V7, Z2, Z4, Fo2].

0.2. The cohomological equation. Our study of the cohomological equation for i.e.m.'s has been prompted by Forni's [Fo1] celebrated paper on the cohomological equation associated to linear flows on surfaces of higher genus. Let us first state our main theorem.

We will denote by $\mathrm{BV}\left(\bigsqcup I_{\alpha}\right)$ (resp. $\mathrm{BV}_{*}\left(\bigsqcup I_{\alpha}\right)$ ) the space of functions $\varphi$ whose restriction to each of the intervals $I_{\alpha}$ is a function of bounded variation (resp. the hyperplane of $\mathrm{BV}\left(\sqcup I_{\alpha}\right)$ made of functions whose integral on the disjoint union $\bigsqcup I_{\alpha}$ vanishes). We will also denote by $\left.\mathrm{BV}_{*}^{1}\left(\bigsqcup I_{\alpha}\right)\right)$ the space of functions $\varphi$ which are absolutely continuous on each $I_{\alpha}$ and whose first derivative belongs to $\mathrm{BV}_{*}\left(\sqcup I_{\alpha}\right)$.

Our first main result can be stated as follows:

Theorem A. Let $T$ be an interval exchange map with the Keane property and of Roth type. Let $\Phi \in B V_{*}^{1}\left(\bigsqcup I_{\alpha}\right)$. There exists a function $\chi$ constant on each interval $I_{\alpha}$ and a bounded function $\Psi$ such that

$$
\Psi-\Psi \circ T=\Phi-\chi .
$$

To make the above statement precise we need to define Roth-type i.e.m.'s. This is the subject of section 1.3 below. For the time being we will content ourselves with briefly describing the three conditions which a Roth-type i.e.m. must satisfy:

(a) The first condition is a growth rate condition for the matrices appearing in an accelerated version of the Zorich continued fraction algorithm (see Section 1.2.4 for details). This condition is the precise analogue of the third of the equivalent arithmetical characterizations of Roth-type irrational numbers given above.

(b) The second condition is a spectral condition which guarantees unique ergodicity of Roth-type i.e.m.'s. This condition does not follow from condition (a) (see Appendix B for a counterexample, and also (Ch) but is automatically satisfied if the i.e.m. is of constant type (i.e. the matrices considered in (a) have bounded norm).

(c) The third and last condition is a coherence condition.

The second main result of this paper is

Theorem B. Roth-type interval exchange maps form a full measure set in the space of all interval exchange maps. 
Obviously, Theorem A is closely connected to Forni's fundamental theorem [Fo1] on the cohomological equation for area-preserving vector fields on surfaces. By singular suspension ("zippered rectangles", see Section 3), one obtains from an interval exchange map an area-preserving flow on a singular flat surface. Forni develops some Fourier analysis tools in this context, which allow him to solve the cohomological equation for almost every direction; our methods are completely different. He works in the Sobolev scale and his methods allow him to lose no more than $3+\varepsilon$ derivatives (for every $\varepsilon>0$ ) [Fo3]. Our loss is smaller and we get an explicit Diophantine condition. On the other hand, given a singular flat surface, we do not know if almost every direction leads to a Roth-type interval exchange map.

The connection with singular flat surfaces explains the type of regularity we introduce when we consider the cohomological equation for more regular data: we still allow discontinuities for $\Phi$ at the endpoints for the $I_{\alpha}$; on the other hand, we require the solution $\Psi$ to be continuous on all of $I$. New linear conditions on $\Phi$ appear by integration of the cohomological equation. See Section 3 below for the precise statements.

When the singular suspension of an i.e.m. $T$ is an invariant foliation for a pseudoAnosov diffeomorphism, the continued fraction expansion of $T$ is eventually periodic. This implies a strong version of condition (a).

Conditions (b) and (c) are also satisfied. Hence $T$ is of Roth type (even of "bounded type") and Theorem A applies. This answers positively a question raised by Forni ([Fo1], p. 342).

0.3. Summary of the contents. In the first section we introduce interval exchange maps and we develop the continued fraction algorithms to an extent which allows us to introduce Roth-type i.e.m.'s. The Keane property (see 1.1.6) does not only guarantee that an i.e.m. is minimal but it also implies that the Rauzy-Veech continued fraction algorithm (described in 1.2.1-1.2.3) can be iterated indefinitely. Accelerating the Rauzy-Veech map by grouping together arrows with the same name in the Rauzy diagram leads to the Zorich continued fraction algorithm (described in 1.2.4) which has the advantage of having a finite mass a.c.i.m. On the other hand, since every name is taken infinitely many times in the sequence of arrows in the Rauzy diagram corresponding to a given i.e.m. one can produce a further acceleration of the scheme by grouping together all arrows which take all possible names but one: this leads to the algorithm we will use in the definition of Roth-type i.e.m.'s given in Section 1.3 and already briefly described above. The notation and the presentation of the Rauzy-Veech-Zorich algorithms follow closely the expository paper $\underline{\mathrm{Y}}$.

Section 2 is devoted to the study of the cohomological equation and to the proof of our main theorem A. When $T$ is a minimal homeomorphism of a compact space $X$, we know from a theorem of Gottschalk and Hedlund $[\mathrm{GH}$ ] that a continuous function on $X$ is a $T$-coboundary of some continuous function as soon as its Birkhoff sums at some point of $X$ are bounded (see Section 2.1.1). An i.e.m. with the Keane property is minimal but not continuous. Nevertheless, a Denjoy-like construction (see Section 2.1.2) allows us to apply Gottschalk-Hedlund's theorem and conclude that a continuous function whose Birkhoff sums at some point are bounded is the $T$-coboundary of a bounded function. The next step in the proof is the reduction of the control of a general Birkhoff sum to the control of those special Birkhoff sums which are obtained by considering the return times of the point under iteration of 
the map (Section 2.2). These can be conveniently analyzed using the continued fraction. The estimates of these special Birkhoff sums for functions of bounded variation are given in Section 2.3 and the proof of the theorem is completed in Section 2.4 .

In Section 3 we first recall how to construct a linear flow on a translation surface starting from an i.e.m. and certain suspension data (Sections 3.1-3.3). Then we relate the discrete cohomological equation for i.e.m.'s to the continuous one for the vertical (area-preserving) vector field constructed by suspension: this allows us to consider more regular data (i.e. belonging to the space $\mathrm{BV}_{*}^{r}$ of functions whose $r$-th derivative has bounded variation on each $I_{\alpha}$ and all intermediate derivatives have zero mean on $\bigsqcup I_{\alpha}$ ). We prove that for those the loss of differentiability in solving the cohomological equation is the same as for functions in $\mathrm{BV}_{*}^{1}$ (Section 3.4).

Section 4 is devoted to the proof of theorem B, i.e. that Roth-type i.e.m.'s have full measure. To this purpose we need to describe how the Rauzy-Veech map acts at the level of the suspension data (Section 4.1). Then we combine the continued fraction algorithm (in Zorich form) with the Teichmüller flow in order to get a version which is normalized w.r.t. scales (Section 4.2). A careful comparison between the a.c.i.m. for the continued fraction map and the Lebesgue measure is carried out in Section 4.3 whereas in Section 4.4, following Zorich [Z1 we prove the integrability condition on the matrices needed to apply Oseledets' multiplicative ergodic theorem. Then conditions (b) and (c) in the definition of Roth-type i.e.m.'s have full measure (Section 4.5) by Oseledets' theorem and the almost sure existence of a spectral gap proved by Veech in V3]. Showing that condition (a) also has full measure requires more work and more precise information on the combinatorics of the continued fraction map. This is summarized in a Proposition stated in Section 4.6 and proved in Section 4.8 whereas in Section 4.3 we show how to conclude the proof of theorem B by putting together the results of Sections 4.3 and 4.7 and applying a Borel-Cantelli argument.

The two appendices are devoted to the construction of concrete examples of Roth-type i.e.m.'s and to the construction of non-uniquely ergodic i.e.m.'s satisfying condition (a) in Roth type (but of course not condition (b)).

\section{The CONTINUED FRACTION ALGORITHM FOR INTERVAL EXCHANGE MAPS}

\subsection{Interval exchange maps.}

1.1.1. An interval exchange map (i.e.m.) is determined by combinatorial data on one side, length data on the other side.

The combinatorial data consists of a finite set $\mathcal{A}$ of names for the intervals and of two bijections $\left(\pi_{0}, \pi_{1}\right)$ from $\mathcal{A}$ onto $\{1, \ldots, d\}$ (where $d$ is the cardinality of $\mathcal{A}$ ): these indicate in which order the intervals are met before and after the map.

The length data $\left(\lambda_{\alpha}\right)_{\alpha \in \mathcal{A}}$ give the length $\lambda_{\alpha}>0$ of the corresponding interval. More precisely, we set

$$
\begin{aligned}
I_{\alpha} & :=\left[0, \lambda_{\alpha}\right) \times\{\alpha\}, \\
\lambda^{*} & :=\sum_{\alpha \in \mathcal{A}} \lambda_{\alpha}, \\
I & :=\left[0, \lambda^{*}\right) .
\end{aligned}
$$


We then define, for $\varepsilon=0,1$, a bijection $j_{\varepsilon}$ from $\bigsqcup_{\alpha \in \mathcal{A}} I_{\alpha}$ onto $I$ :

$$
j_{\varepsilon}(x, \alpha)=\sum_{\pi_{\varepsilon}(\beta)<\pi_{\varepsilon}(\alpha)} \lambda_{\beta}+x .
$$

The i.e.m. $T$ associated to these data is the bijection $T=j_{1} \circ j_{0}^{-1}$ of $I$.

1.1.2. If $\mathcal{A}, \pi_{0}, \pi_{1}, \lambda_{\alpha}$ are as above and $\chi: \mathcal{A}^{\prime} \rightarrow \mathcal{A}$ is a bijection, we can define a new set of data by

$$
\begin{aligned}
\pi_{\varepsilon}^{\prime} & =\pi_{\varepsilon} \circ \chi, \varepsilon=0,1, \\
\lambda_{\alpha^{\prime}}^{\prime} & =\lambda_{\chi\left(\alpha^{\prime}\right)}, \alpha^{\prime} \in \mathcal{A}^{\prime} .
\end{aligned}
$$

Obviously, the new i.e.m. $T^{\prime}$ determined by these data is the same, except for names, as the old one. In particular, we could restrict our attention to normalized combinatorial data characterized by

$$
\mathcal{A}=\{1, \ldots, d\}, \pi_{0}=\operatorname{id}_{\mathcal{A}} .
$$

However, this leads later to more complicated formulas in the continued fraction algorithm because the basic operations on i.e.m.'s do not preserve normalization.

1.1.3. Given combinatorial data $\left(\mathcal{A}, \pi_{0}, \pi_{1}\right)$, we set, for $\alpha, \beta \in \mathcal{A}$,

$$
\Omega_{\alpha, \beta}= \begin{cases}+1 & \text { if } \pi_{0}(\beta)>\pi_{0}(\alpha), \pi_{1}(\beta)<\pi_{1}(\alpha), \\ -1 & \text { if } \pi_{0}(\beta)<\pi_{0}(\alpha), \pi_{1}(\beta)>\pi_{1}(\alpha), \\ 0 & \text { otherwise. }\end{cases}
$$

The matrix $\Omega=\left(\Omega_{\alpha, \beta}\right)_{(\alpha, \beta) \in \mathcal{A}^{2}}$ is antisymmetric.

Let $\left(\lambda_{\alpha}\right)_{\alpha \in \mathcal{A}}$ be the length data and let $T$ be the associated i.e.m. For $\alpha \in \mathcal{A}$, $y \in j_{0}\left(I_{\alpha}\right)$, we have

$$
T(y)=y+\delta_{\alpha},
$$

where the translation vector $\delta=\left(\delta_{\alpha}\right)_{\alpha \in \mathcal{A}}$ is related to the length vector $\lambda=\left(\lambda_{\alpha}\right)_{\alpha \in \mathcal{A}}$ by

$$
\delta=\Omega \lambda .
$$

1.1.4. There is a canonical involution $\mathcal{I}$ acting on the set of combinatorial data which exchange $\pi_{0}$ and $\pi_{1}$. For any set $\left(\lambda_{\alpha}\right)_{\alpha \in \mathcal{A}}$ of length data, the intervals $I_{\alpha}, I$ are unchanged, but $j_{0}$ and $j_{1}$ are exchanged and $T$ is replaced by $T^{-1}$. The matrix $\Omega$ is replaced by $-\Omega$ and the translation vector $\delta$ by $-\delta$. Observe that $\mathcal{I}$ does not respect the combinatorial normalization.

1.1.5. In the following, we will always consider only combinatorial data $\left(\mathcal{A}, \pi_{0}, \pi_{1}\right)$ which are admissible, meaning that for all $k=1,2, \ldots, d-1$, we have

$$
\pi_{0}^{-1}(\{1, \ldots, k\}) \neq \pi_{1}^{-1}(\{1, \ldots, k\}) .
$$

Indeed, if we had $\pi_{0}^{-1}(\{1, \ldots, k\})=\pi_{1}^{-1}(\{1, \ldots, k\})$ for some $k<d$, for any length data $\left(\lambda_{\alpha}\right)_{\alpha \in \mathcal{A}}$, the interval $I$ would decompose into two disjoint invariant subintervals and the study of the dynamics would be reduced to simpler combinatorial data. 
1.1.6. The Keane property. Let $T$ be an i.e.m. defined by combinatorial data $\left(\mathcal{A}, \pi_{0}, \pi_{1}\right)$ and length data $\left(\lambda_{\alpha}\right)_{\alpha \in \mathcal{A}}$.

Definition. A connection for $T$ is a triple $(\alpha, \beta, m)$ where $\alpha, \beta \in \mathcal{A}, \pi_{0}(\beta)>1, m$ is a positive integer, and

$$
T^{m}\left(j_{0}(0, \alpha)\right)=j_{0}(0, \beta) .
$$

We say that $T$ has the Keane property if there is no connection for $T$.

It turns out that this property is the appropriate notion of irrationality for i.e.m. The following results are due to Keane (Ke1]):

- An i.e.m. with Keane's property is minimal (i.e. all orbits are dense).

- If the length data are rationally independent (and the combinatorial data are admissible), then $T$ has Keane's property.

\subsection{The continued fraction algorithm.}

1.2.1. The basic operation (Rauzy $\mathrm{Ra}]$, Veech $[\mathrm{V} 2]$ ). Let $T$ be an i.e.m. defined by combinatorial data $\left(\mathcal{A}, \pi_{0}, \pi_{1}\right)$ and length data $\left(\lambda_{\alpha}\right)_{\alpha \in \mathcal{A}}$. We assume as always that the combinatorial data are admissible.

We denote by $\alpha_{0}, \alpha_{1}$ the (distinct) elements of $\mathcal{A}$ such that

$$
\pi_{0}\left(\alpha_{0}\right)=\pi_{1}\left(\alpha_{1}\right)=d .
$$

Observe that if $\lambda_{\alpha_{0}}=\lambda_{\alpha_{1}}$, the triple $\left(\alpha_{0}, \alpha_{1}, 1\right)$ is a connection and $T$ has not the Keane property.

We now assume that $\lambda_{\alpha_{0}} \neq \lambda_{\alpha_{1}}$ and define $\varepsilon \in\{0,1\}$ by

$$
\lambda_{\alpha_{\varepsilon}}=\operatorname{Max}\left(\lambda_{\alpha_{0}}, \lambda_{\alpha_{1}}\right) \text {. }
$$

We set

$$
\begin{aligned}
\hat{\lambda}^{*} & =\lambda^{*}-\lambda_{\alpha_{1-\varepsilon}}, \\
\hat{I} & =\left[0, \hat{\lambda}^{*}\right) \subset I,
\end{aligned}
$$

and define $\hat{T}: \hat{I} \rightarrow \hat{I}$ to be the first return map of $T$ in $\hat{I}$.

When $\varepsilon=0$ we have

$$
\hat{T}(y)= \begin{cases}T(y) & \text { if } y \notin j_{0}\left(I_{\alpha_{1}}\right), \\ T^{2}(y) & \text { if } y \in j_{0}\left(I_{\alpha_{1}}\right) .\end{cases}
$$

When $\varepsilon=1$ we have similarly

$$
\hat{T}^{-1}(y)= \begin{cases}T^{-1}(y) & \text { if } y \notin j_{1}\left(I_{\alpha_{0}}\right), \\ T^{-2}(y) & \text { if } y \in j_{1}\left(I_{\alpha_{0}}\right) .\end{cases}
$$

In both cases, it appears that $\hat{T}$ is again an interval exchange map which can be defined using the same alphabet $\mathcal{A}$. The length data for $\hat{T}$ are given by

$$
\begin{gathered}
\hat{\lambda}_{\alpha}=\lambda_{\alpha} \text { if } \alpha \neq \alpha_{\varepsilon}, \\
\hat{\lambda}_{\alpha_{\varepsilon}}=\lambda_{\alpha_{\varepsilon}}-\lambda_{\alpha_{1-\varepsilon}} .
\end{gathered}
$$

The combinatorial data $\left(\hat{\pi}_{0}, \hat{\pi}_{1}\right)$ for $\hat{T}$ are given by

$$
\hat{\pi}_{\varepsilon}=\pi_{\varepsilon}
$$




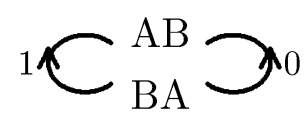

$d=2$

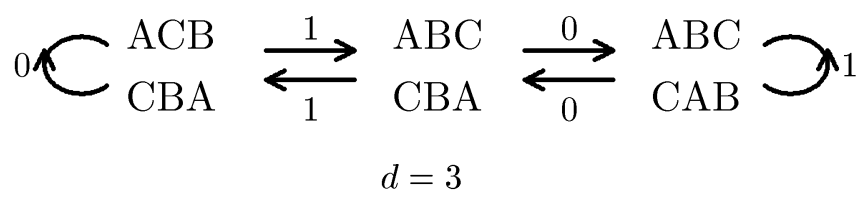

FiguRE 1. Rauzy diagrams $d=2$ and 3

and

$$
\hat{\pi}_{1-\varepsilon}(\alpha)= \begin{cases}\pi_{1-\varepsilon}(\alpha) & \text { if } \pi_{1-\varepsilon}(\alpha) \leq \pi_{1-\varepsilon}\left(\alpha_{\varepsilon}\right), \\ \pi_{1-\varepsilon}(\alpha)+1 & \text { if } \pi_{1-\varepsilon}\left(\alpha_{\varepsilon}\right)<\pi_{1-\varepsilon}(\alpha)<d, \\ \pi_{1-\varepsilon}\left(\alpha_{\varepsilon}\right)+1 & \text { if } \pi_{1-\varepsilon}(\alpha)=d .\end{cases}
$$

We rewrite the relation between old and new length data as

$$
\lambda=V \hat{\lambda},
$$

where

$$
V=\mathbf{I}+E_{\alpha_{\varepsilon} \alpha_{1-\varepsilon}}
$$

has now nonnegative integer coefficients and belongs to the group SL $\left(\mathbb{Z}^{\mathcal{A}}\right)$. We also write

$$
\left(\hat{\pi}_{0}, \hat{\pi}_{1}\right)=R_{\varepsilon}\left(\pi_{0}, \pi_{1}\right)
$$

and observe that these new combinatorial data are admissible.

1.2.2. Rauzy diagrams. Let $\mathcal{A}$ be an alphabet. We define an oriented graph as follows. The vertices are the admissible pairs $\left(\pi_{0}, \pi_{1}\right)$. Each vertex $\left(\pi_{0}, \pi_{1}\right)$ is the starting point of exactly two arrows with endpoints at $R_{0}\left(\pi_{0}, \pi_{1}\right)$ and $R_{1}\left(\pi_{0}, \pi_{1}\right)$. The arrow connecting $\left(\pi_{0}, \pi_{1}\right)$ to $R_{\varepsilon}\left(\pi_{0}, \pi_{1}\right)$ is said to be of type $\varepsilon$.

The operations $R_{0}, R_{1}$ are obviously invertible. Therefore each vertex is also the endpoint of exactly two arrows, one of each type.

To each arrow in the graph, we associate a name in $\mathcal{A}$ : it is the element $\alpha_{\varepsilon}$ such that $\pi_{\varepsilon}\left(\alpha_{\varepsilon}\right)=d$ (where $\left(\pi_{0}, \pi_{1}\right)$ is the starting point of the arrow and $\varepsilon$ is its type). The element $\alpha_{1-\varepsilon}$ will then be called the secondary name of this arrow. A Rauzy diagram is a connected component of this oriented graph.

Obviously, the Rauzy operations $R_{0}, R_{1}$ commute with a change of names (see 1.2). Up to a change of names, there is only one Rauzy diagram with $d=\operatorname{card} \mathcal{A}=$ 2 , and one with $d=\operatorname{card} \mathcal{A}=3$ (see Figure 1).

In the diagrams above the pair $\left(\pi_{0}, \pi_{1}\right)$ is denoted by the symbol

$$
\begin{array}{lll}
\pi_{0}^{-1}(1) & \ldots & \pi_{0}^{-1}(d), \\
\pi_{1}^{-1}(1) & \ldots & \pi_{1}^{-1}(d) .
\end{array}
$$

For $d=\operatorname{card} \mathcal{A}=4$ there are 2 distinct Rauzy diagrams: see Figure 2 ,

In each of these diagrams, the symmetry with respect to the vertical axis corresponds to the action of the canonical involution. 
$0 \smile \begin{gathered}\mathrm{ACDB} \\ \mathrm{DCBA}\end{gathered}$
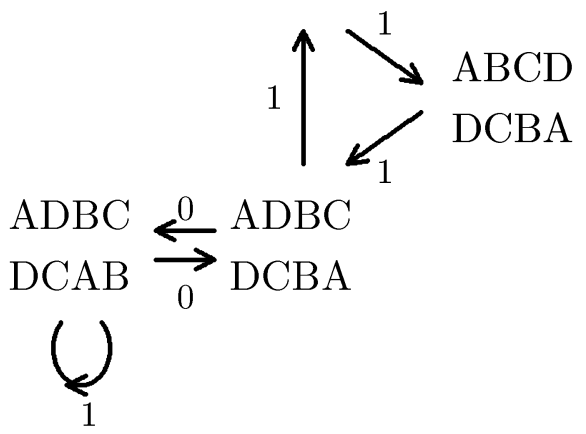
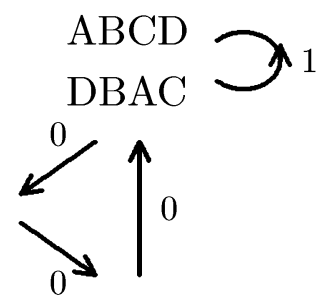

$\mathrm{ABCD}$

$\mathrm{DACB}$
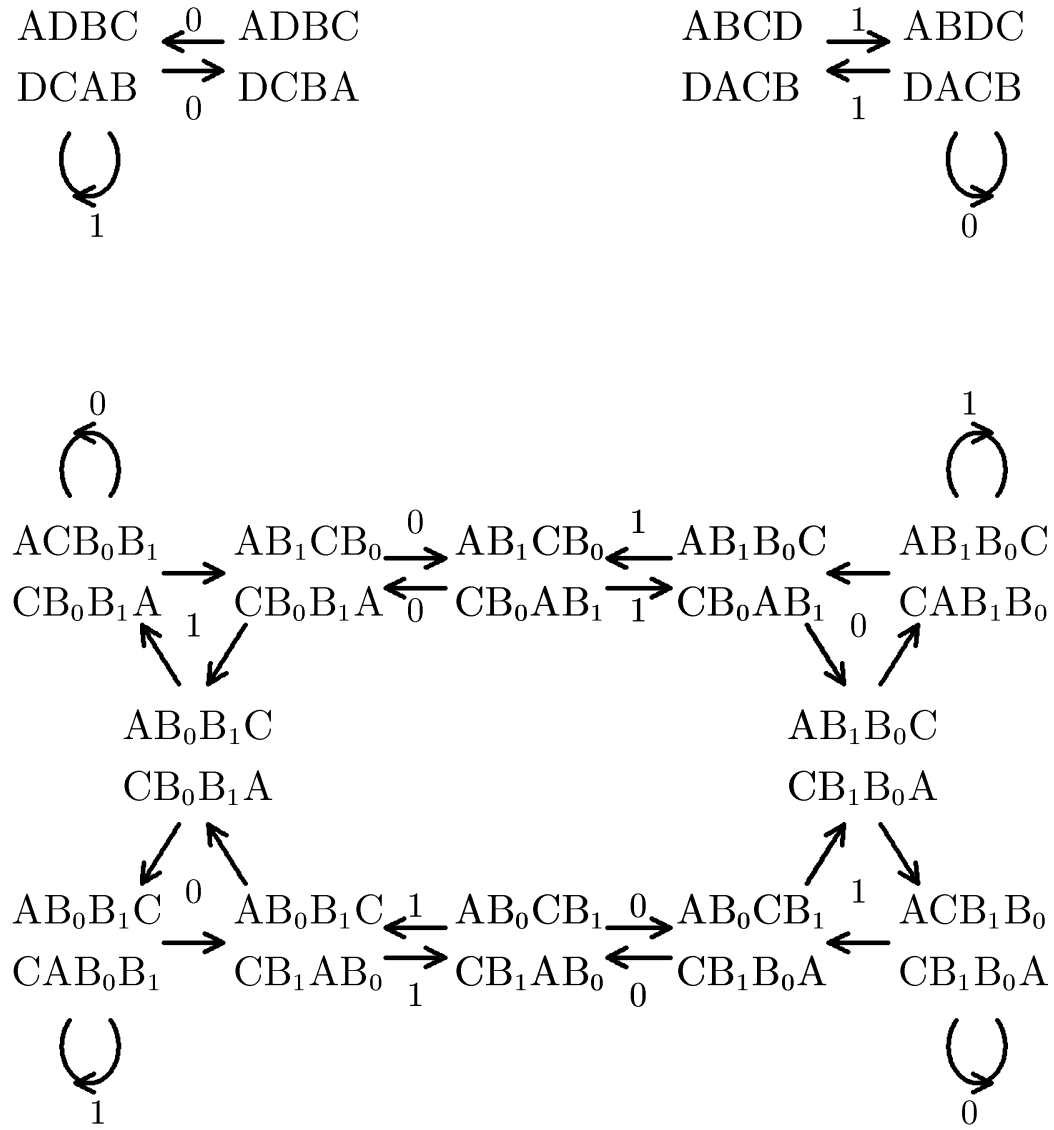

FiguRE 2. Rauzy diagrams for $d=4$

In the last diagram, there is a further symmetry with respect to the center of the diagram, which corresponds to the exchange of the names $B_{0}, B_{1}$. This is a monodromy phenomenon: to each admissible pair $\left(\pi_{0}, \pi_{1}\right)$, one can associate the permutation $\pi:=\pi_{1} \circ \pi_{0}^{-1}$ of $\{1, \ldots, d\}$, which is invariant under a change of names. When we identify vertices with the same permutation, we obtain a reduced Rauzy diagram and we have a covering map from the Rauzy diagram onto the reduced Rauzy diagram.

In the first three examples above, the covering map is an isomorphism. In the last example, the degree of the covering map is 2 and the reduced Rauzy diagram is given in Figure 3, where $\pi$ is denoted by $\left(\pi^{-1}(1), \ldots, \pi^{-1}(d)\right)$. 


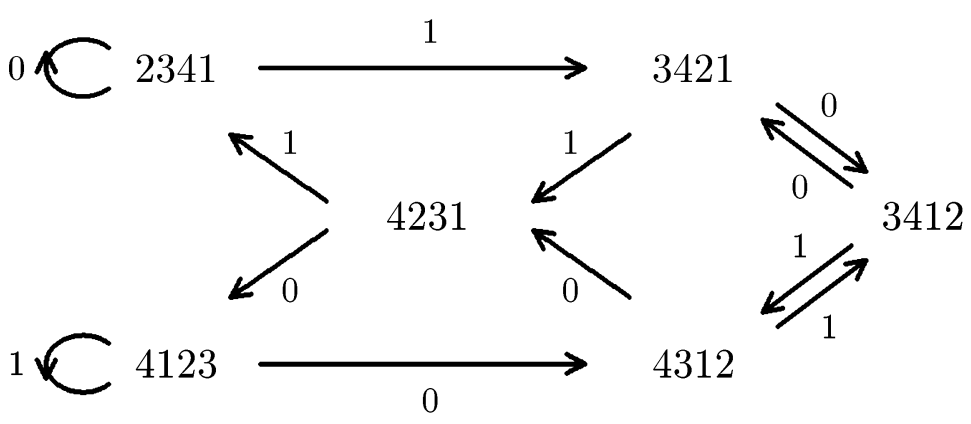

FiguRE 3. Reduced Rauzy diagram for $d=4$

1.2.3. The Rauzy-Veech algorithm. Let $T$ be an i.e.m. with admissible combinatorial data. If $T$ has Keane's property, the basic operation is defined for $T$ and it is immediate to check that the new i.e.m. $\hat{T}$ again has Keane's property. Therefore we can iterate the basic operation and generate a sequence $\left(T^{(n)}\right)_{n \geq 0}$ of i.e.m.'s (with $T^{(0)}=T$ ). We will denote by $\left(\pi_{0}^{(n)}, \pi_{1}^{(n)}\right)$ the combinatorial data of $T^{(n)}$, by $\left(\lambda_{\alpha}^{(n)}\right)_{\alpha \in \mathcal{A}}$ its length data, by $\gamma^{(n)}$ the arrow in the Rauzy diagram connecting $\left(\pi_{0}^{(n-1)}, \pi_{1}^{(n-1)}\right)$ to $\left(\pi_{0}^{(n)}, \pi_{1}^{(n)}\right)$, by $V^{(n)}$ the matrix relating $\lambda^{(n-1)}$ to $\lambda^{(n)}$ through

$$
\lambda^{(n-1)}=V^{(n)} \lambda^{(n)} .
$$

Conversely, it is not difficult to check that when $T$ has a connection, the algorithm has to stop because, at some point in the equality, one runs into the case $\lambda_{\alpha_{0}}=\lambda_{\alpha_{1}}$ in the basic operation.

Proposition. Each name in $\mathcal{A}$ is taken infinitely many times by the sequence of arrows $\left(\gamma^{(n)}\right)_{n>0}$.

Proof. Let $\mathcal{A}^{\prime}$ be the set of names which are taken infinitely many times and let $\mathcal{A}=\mathcal{A} \backslash \mathcal{A}^{\prime}$. Replacing $T$ by some $T^{(N)}$, we can assume that names in $\mathcal{A}^{\prime \prime}$ are not taken at all. Then the lengths $\lambda_{\alpha}^{(n)}, \alpha \in \mathcal{A}^{\prime \prime}$, do not depend on $n$. But then elements $\alpha \in \mathcal{A}^{\prime \prime}$ can only appear as secondary names at most finitely many times. Replacing again $T$ by some $T^{(N)}$, we can assume that secondary names are never in $\mathcal{A}^{\prime \prime}$. Then the sequences $\left(\pi_{\varepsilon}^{(n)}(\alpha)\right)_{n>0}$, for $\varepsilon \in\{0,1\}, \alpha \in \mathcal{A}^{\prime \prime}$, are nondecreasing and we can assume (replacing once again $T$ by some $T^{(N)}$ ) that they are constant.

We now claim that we must have $\pi_{\varepsilon}^{(0)}\left(\alpha^{\prime \prime}\right)<\pi_{\varepsilon}^{(0)}\left(\alpha^{\prime}\right)$ for all $\alpha^{\prime \prime} \in \mathcal{A}^{\prime \prime}, \alpha^{\prime} \in \mathcal{A}^{\prime}$ and $\varepsilon \in\{0,1\}$. Because the pair $\left(\pi_{0}^{(0)}, \pi_{1}^{(0)}\right)$ is admissible, this implies $\mathcal{A}^{\prime}=\mathcal{A}$. To prove the claim, assume that there exist $\alpha^{\prime} \in \mathcal{A}^{\prime}, \alpha^{\prime \prime} \in \mathcal{A}^{\prime \prime}, \varepsilon \in\{0,1\}$ with $\pi_{\varepsilon}^{(0)}\left(\alpha^{\prime}\right)<\pi_{\varepsilon}^{(0)}\left(\alpha^{\prime \prime}\right)$. As $\pi_{\varepsilon}^{(n)}\left(\alpha^{\prime \prime}\right)=\pi_{\varepsilon}^{(0)}\left(\alpha^{\prime \prime}\right)$ for all $n \geq 0$, we can never have $\pi_{\varepsilon}^{(n)}\left(\alpha^{\prime}\right)=d$ for some $n>0$. By definition of $\mathcal{A}^{\prime}$, there must exist $n \geq 0$ such that $\pi_{1-\varepsilon}^{(n)}\left(\alpha^{\prime}\right)=d$; but then $\pi_{\varepsilon}^{(n+1)}\left(\alpha^{\prime \prime}\right) \neq \pi_{\varepsilon}^{(0)}\left(\alpha^{\prime \prime}\right)$, which gives a contradiction.

1.2.4. The Zorich algorithm and its accelerations. When $d=2$, setting $x=\lambda_{B} / \lambda_{A}$, the basic operation reduces to the well-known map

$$
g(x)= \begin{cases}\frac{x}{1-x} & \text { for } 0<x<1 / 2 \\ \frac{1-x}{x} & \text { for } 1 / 2<x<1\end{cases}
$$


with a parabolic fixed point at 0 . There is a unique absolutely continuous invariant measure, namely $d x / x$, but this measure is infinite. On the other hand, the Gauss map generating the continued fraction algorithm has $d x /(1+x)$ as a finite a.c.i.m.

For i.e.m.'s with more intervals, identifying i.e.m.'s with proportional length data (and the same combinatorial data), Veech has shown V2 that there exists again for the basic operation a unique absolutely continuous invariant measure. Again this measure is infinite. Zorich has discovered ([Z1]) how to concatenate several steps of the basic operations in order to get a finite a.c.i.m.

Let $T$ be an i.e.m. with Keane's property, $T^{(n)}, \gamma^{(n)}, V^{(n)}$ the data generated by the iteration of the basic operation. Let also $1 \leq D<d$. We define inductively an increasing sequence $n_{D}(k)=n_{D}(k, T)$ by setting $n_{D}(0)=0$ and where $n_{D}(k+1)$ is the largest integer such that no more than $D$ names are taken by the $\gamma^{(n)}$, for $n_{D}(k)<n \leq n_{D}(k+1)$.

The sequence is well defined because of the Proposition above.

Obviously, for $1<D<d,\left(n_{D}(k)\right)_{k \geq 0}$ is a subsequence of $\left(n_{D-1}(l)\right)_{l \geq 0}$.

We will define, for $k>0$,

$$
Z_{(D)}(k)=V^{\left(n_{D}(k-1)+1\right)} \cdots V^{\left(n_{D}(k)\right)} .
$$

The case $D=1$ is the one considered by Zorich ([Z1]). We will on the other hand be interested in the case $D=d-1$.

When the context is clear, we will simply write $Z(k)$ for $Z_{(d-1)}(k)$ and $T^{(k)}$ for $T^{\left(n_{d-1}(k)\right)}, \lambda^{(k)}$ for its length data. With this notation, we have

$$
\lambda^{(k)}=Z(k+1) \lambda^{(k+1)} .
$$

We will also set, for $k<l$,

$$
Q(k, l)=Z(k+1) \cdots Z(l),
$$

in order to have

$$
\lambda^{(k)}=Q(k, l) \lambda^{(l)} .
$$

We will also write $Q(l)$ for $Q(0, l)$. The coefficients $Q_{\alpha \beta}(k, l)$ have the following interpretation. Let $I^{(k)}=\bigsqcup_{\alpha \in \mathcal{A}} j_{0}\left(I_{\alpha}^{(k)}\right)$ be the domain of $T^{(k)}$. For $l \geq k$, we have $I^{(l)} \subset I^{(k)}$ and $T^{(l)}$ is the first return map of $T^{(k)}$ in $I^{(l)}$. Then, the nonnegative integer $Q_{\alpha \beta}(k, l)$ is the time spent in $j_{0}\left(I_{\alpha}^{(k)}\right)$ by any point of $j_{0}\left(I_{\beta}^{(l)}\right)$ until it returns in $I^{(l)}$.

We will also introduce

$$
Q_{\beta}(k, l)=\sum_{\alpha \in \mathcal{A}} Q_{\alpha \beta}(k, l),
$$

which is the return time in $I^{(l)}$ for points in $I_{\beta}^{(l)}$.

The following lemma is the main reason to choose $D=d-1$ rather than $D=1$.

Lemma. Let $T$ satisfy Keane's condition. Assume that

$$
l \geq \begin{cases}k+2 d-3 & \text { if } d \geq 3, \\ k+2 & \text { if } d=2 .\end{cases}
$$

Then, for all $\alpha, \beta \in \mathcal{A}$, we have $Q_{\alpha \beta}(k, l)>0$. 
Proof. Replacing $T$ by $T^{(k)}$, it is sufficient to consider the case $k=0$. For $r \geq 0$, set

$$
\hat{Q}(r)=V^{(1)} \cdots V^{(r)}
$$

as the diagonal terms of the $V$ matrices are equal to 1 (and all the terms are nonnegative) we have

$$
\hat{Q}_{\alpha \beta}(r)>0 \Rightarrow \hat{Q}_{\alpha \beta}(r+1)>0 .
$$

Fix $\alpha, \beta \in \mathcal{A}$. We will construct a sequence of distinct indices $\alpha_{1}=\alpha, \alpha_{2}, \ldots, \alpha_{s}$ $=\beta$ and integers $r_{1}=0<r_{2}<\ldots<r_{s}$ such that

$$
\hat{Q}_{\alpha_{1} \alpha_{j}}(r)>0 \text { for } r \geq r_{j} .
$$

If $\alpha=\beta$, then $s=1, r_{1}=0$ and the property is satisfied. Otherwise, let $r_{2}$ be the smallest positive integer such that the name of $\gamma^{\left(r_{2}\right)}$ is $\alpha_{1}$, and let $\alpha_{2}$ be the secondary name of $\gamma^{\left(r_{2}\right)}$; we have $\alpha_{2} \neq \alpha_{1}$ and $V_{\alpha_{1} \alpha_{2}}^{\left(r_{2}\right)}=1$; hence $\hat{Q}_{\alpha_{1} \alpha_{2}}(r)>0$ for $r \geq r_{2}$.

Assume that $\alpha_{1}, \ldots, \alpha_{j}, r_{1}, \ldots, r_{j}$ have been constructed, with $\beta \neq \alpha_{l}$ for $1 \leq$ $l \leq j$. Let $r_{j}^{\prime}$ be the smallest integer $>r_{j}$ such that the name of $\gamma^{\left(r_{j}^{\prime}\right)}$ does not belong to $\left\{\alpha_{1}, \ldots, \alpha_{j}\right\}$ and let $r_{j+1}$ be the smallest integer $>r_{j}^{\prime}$ such that the name of $\gamma^{\left(r_{j+1}\right)}$ belongs to $\left\{\alpha_{1}, \ldots, \alpha_{j}\right\}$; let $\alpha_{j+1}$ be the secondary name of $\gamma^{\left(r_{j+1}\right)}$. Then $\alpha_{j+1}$ is the name of $\gamma^{\left(r_{j+1}-1\right)}$ and therefore is distinct from $\alpha_{1}, \ldots, \alpha_{j}$. By construction, we have, for some $1 \leq l \leq j$,

$$
V_{\alpha_{l} \alpha_{j+1}}^{\left(r_{j+1}\right)}=1
$$

and also

$$
\hat{Q}_{\alpha_{1} \alpha_{l}}\left(r_{j+1}-1\right)>0
$$

because $r_{j+1}>r_{l}$. We conclude that

$$
\hat{Q}_{\alpha_{1} \alpha_{j+1}}(r)>0 \text { for } r \geq r_{j+1} .
$$

At some point we will obtain $\alpha_{s}=\beta$. It remains to see how many steps of the accelerated Zorich algorithm (with $D=d-1$ ) are needed to attain $r_{s}$. Obviously, we have $r_{2} \leq n_{d-1}(1)+1$. Then, for $2 \leq j<d-1$, we have

$$
\begin{aligned}
r_{j}^{\prime} & \leq n_{d-1}(2 j-2), \\
r_{j+1} & \leq n_{d-1}(2 j-1) .
\end{aligned}
$$

Finally, when $s=d>2$, we have

$$
\begin{aligned}
r_{d-1}^{\prime} & \leq n_{d-1}(2 d-4)+1 \\
r_{d} & \leq n_{d-1}(2 d-3) .
\end{aligned}
$$

1.3. Roth-type interval exchange maps. Roth-type i.e.m.'s should satisfy Keane's condition so that the continued fraction algorithm is defined, and three further conditions which are now explained. 
1.3.1. Size of the $Z$ matrices. Take $D=d-1$ in 1.2.4. We will first ask for the $Z$ matrices to be not too big in the following sense:

(a) for every $\varepsilon>0$ there exists $C_{\varepsilon}>0$ such that for all $k \geq 0$ we have

$$
\|Z(k+1)\| \leq C_{\varepsilon}\|Q(k)\|^{\varepsilon} .
$$

When $d=2$, this amounts exactly to the classical Roth-type approximation property for an irrational number $\theta$ : for all $\varepsilon>0$, there exists $\gamma_{\varepsilon}>0$ such that for all rational $p / q$ one has

$$
|\theta-p / q| \geq \gamma_{\varepsilon} q^{-2-\varepsilon} .
$$

In terms of the convergents $\left(p_{k} / q_{k}\right)_{k \geq 0}$ of $\theta$ with partial quotients $\left(a_{k}\right)_{k \geq 1}$, this is equivalent to having, for all $\varepsilon>0$,

$$
a_{k+1}=\mathrm{O}\left(q_{k}^{\varepsilon}\right) \text {, }
$$

which explains our terminology.

We can reformulate (a) in terms of the lengths $\lambda_{\alpha}^{(k)}$. It is convenient here to take as the norm of a matrix the sum of all coefficients (in absolute value; the matrices that we consider here have nonnegative entries).

Proposition. We have always, for $k \geq 0$,

$$
\operatorname{Max}_{\alpha \in \mathcal{A}} \lambda_{\alpha}^{(k)} \geq \lambda^{*}\|Q(k)\|^{-1} \geq \operatorname{Min}_{\alpha \in \mathcal{A}} \lambda_{\alpha}^{(k)} .
$$

Condition (a) is equivalent to the following converse estimate: for all $\varepsilon>0$, there exists $C_{\varepsilon}>0$ such that

$$
\operatorname{Max}_{\alpha \in \mathcal{A}} \lambda_{\alpha}^{(k)} \leq C_{\varepsilon} \operatorname{Min}_{\alpha \in \mathcal{A}} \lambda_{\alpha}^{(k)}\|Q(k)\|^{\varepsilon} .
$$

Proof. The first estimate follows from

$$
\lambda^{*}=\sum_{\alpha \in \mathcal{A}} \lambda_{\alpha}^{(0)}=\sum_{\beta \in \mathcal{A}} Q_{\beta}(k) \lambda_{\beta}^{(k)} .
$$

Assume (a) is satisfied. Let $l$ be equal to $k+2 d-3$ (if $d \geq 3$ ) or $k+2$ (if $d=2$ ) as in the lemma in 1.2.4. We have

$$
\|Q(k, l)\| \leq C_{\varepsilon}^{\prime}\|Q(k)\|^{\varepsilon}
$$

for all $\varepsilon>0$ (with an appropriate constant $C_{\varepsilon}^{\prime}$ ).

This gives

$$
\operatorname{Max}_{\alpha \in \mathcal{A}} \lambda_{\alpha}^{(k)} \leq C_{\varepsilon}^{\prime}\|Q(k)\|^{\varepsilon} \operatorname{Max}_{\alpha \in \mathcal{A}} \lambda_{\alpha}^{(l)} .
$$

On the other hand, Lemma 1.2.4 gives

$$
\operatorname{Min}_{\alpha \in \mathcal{A}} \lambda_{\alpha}^{(k)} \geq \operatorname{Max}_{\alpha \in \mathcal{A}} \lambda_{\alpha}^{(l)},
$$

giving the required estimate. Assume now that the estimate of the proposition holds. We have always

$$
\operatorname{Max}_{\alpha \in \mathcal{A}} \lambda_{\alpha}^{(k)} \geq d^{-1}\|Z(k+1)\| \operatorname{Min}_{\alpha \in \mathcal{A}} \lambda_{\alpha}^{(k+1)} .
$$

On the other hand, by the definition of the $Z$ matrices, there exists $\alpha_{0} \in \mathcal{A}$ such that

$$
\lambda_{\alpha_{0}}^{(k)}=\lambda_{\alpha_{0}}^{(k+1)} .
$$


But we have

$$
\begin{aligned}
\lambda_{\alpha_{0}}^{(k+1)} & \leq \operatorname{Max}_{\alpha \in \mathcal{A}} \lambda_{\alpha}^{(k+1)} \\
& \leq C_{\varepsilon} \operatorname{Min}_{\alpha \in \mathcal{A}} \lambda_{\alpha}^{(k+1)}\|Q(k+1)\|^{\varepsilon} \\
& \leq C_{\varepsilon} d\|Z(k+1)\|^{-1} \operatorname{Max}_{\alpha \in \mathcal{A}} \lambda_{\alpha}^{(k)}\|Q(k+1)\|^{\varepsilon} \\
& \leq C_{\varepsilon}^{2} d\|Z(k+1)\|^{-1} \operatorname{Min}_{\alpha \in \mathcal{A}} \lambda_{\alpha}^{(k)}\|Q(k)\|^{\varepsilon}\|Q(k+1)\|^{\varepsilon} \\
& \leq C_{\varepsilon}^{2} d\|Z(k+1)\|^{-1} \lambda_{\alpha_{0}}^{(k)}\|Q(k)\|^{\varepsilon}\|Q(k+1)\|^{\varepsilon},
\end{aligned}
$$

which implies

$$
\|Z(k+1)\| \leq C_{\varepsilon}^{2} d\|Q(k)\|^{\varepsilon}\|Q(k+1)\|^{\varepsilon}
$$

and allows us to conclude that (a) holds.

Remark 1. Assume condition (a) is satisfied. Set $k_{0}=2 d-3$ if $d \geq 3, k_{0}=2$ if $d=2$. Following the same lines as in the last proposition, we see that for any $\varepsilon>0$, there exists $C_{\varepsilon}>0$ such that for $k \geq k_{0}$ we have

$$
\operatorname{Min}_{\alpha, \beta \in \mathcal{A}} Q_{\alpha \beta}(k) \geq C_{\varepsilon}^{-1}\|Q(k)\|^{1-\varepsilon} .
$$

On the other hand it is easy to see that, even in the case of 3 intervals, this estimate does not imply condition (a).

Remark 2. Boshernitzan has defined ([B] $)$ another condition which generalizes the Roth condition for irrational numbers. Namely, he asks that $T$ satisfy Keane's condition and that the minimum distance $m_{n}$ between discontinuity points of the $n$-th iterate $T^{n}$ of $T$ should satisfy

$$
m_{n} \geq \frac{\gamma_{\varepsilon}^{-1}}{n^{1+\varepsilon}}
$$

He proves that this condition has full measure.

The relation between Boshernitzan's condition and condition (a) above is however not clear.

1.3.2. Spectral gap. As soon as $k \geq 2 d-3(k \geq 2$ if $d=2)$, all entries in the matrix $Q(k)$ are strictly positive. It is therefore not unreasonable to expect that the positive cone is more expanded by $Q(k)$ than the other directions, in the spirit of the Perron-Frobenius theorem.

However this is not automatic, as attested to by the existence of minimal nonuniquely ergodic i.e.m.'s (an i.e.m. satisfying Keane's condition is uniquely ergodic if and only if the image under $Q(k)$ of the positive cone converges to a ray as $k \rightarrow \infty)$.

Our second condition ensures that this weird behaviour does not occur.

For each $k \geq 0$, let $\Gamma^{(k)}$ be a copy of $\mathbb{R}^{\mathcal{A}}$. One should think of $\Gamma^{(k)}$ as the space of functions on $\bigsqcup_{\alpha \in \mathcal{A}} I_{\alpha}^{(k)}$ which are constant on each $I_{\alpha}^{(k)}$. For $0 \leq k \leq l$, let $S(k, l)$ be the linear map from $\Gamma^{(k)}$ to $\Gamma^{(l)}$ whose matrix in the canonical basis is ${ }^{t} Q(k, l)$. This can be interpreted as a special Birkhoff sum (see Section 2 below).

For $\varphi=\left(\varphi_{\alpha}\right)_{\alpha \in \mathcal{A}} \in \Gamma^{(k)}$, define

$$
I_{k}(\varphi)=\sum_{\alpha \in \mathcal{A}} \lambda_{\alpha}^{(k)} \varphi_{\alpha}
$$

we have then

$$
I_{l}(S(k, l) \varphi)=I_{k}(\varphi)
$$


Denote by $\Gamma_{*}^{(k)}$ the kernel of the linear form $I_{k}$. We will ask the following:

(b) There exists $\theta>0, C>0$ such that, for all $k \geq 0$, we have

$$
\left\|\left.S(k)\right|_{\Gamma_{*}^{(0)}}\right\| \leq C\|S(k)\|^{1-\theta}=C\|Q(k)\|^{1-\theta} .
$$

Observe that an i.e.m. satisfying Keane's condition and (b) must be uniquely ergodic.

In appendix B we construct i.e.m.'s which satisfy condition (a) but are not uniquely ergodic (see also $\mathrm{Ch}]$ ); therefore condition (b) is not a consequence of condition (a).

However, if instead of condition (a) we consider the stronger condition (being reminded of bounded-type irrational numbers):

(ã) the sequence $Z(k)$ is bounded, then condition (b) follows. Indeed, each $Q(k, k+2 d-3)(Q(k, k+2)$ when $d=2)$ will contract by a definite factor $<1$ the Hilbert metric of the projective positive cone.

1.3.3. Coherence. To define our third condition, we consider again the operators $S(k, l): \Gamma^{(k)} \rightarrow \Gamma^{(l)}$. Let $\Gamma_{s}^{(k)}$ be the linear subspace of $\Gamma^{(k)}$ whose elements $v$ satisfy the following: there exists $\sigma=\sigma(v)>0, C=C(v)>0$ such that, for all $l \geq k$, one has

$$
\|S(k, l) v\| \leq C\|S(k, l)\|^{-\sigma}\|v\| .
$$

We call $\Gamma_{s}^{(k)}$ the stable subspace of $\Gamma^{(k)}$. Obviously, one has $\Gamma_{s}^{(k)} \subset \Gamma_{*}^{(k)}$. On the other hand, $\Gamma_{s}^{(k)}$ is never reduced to 0 because it always contains the translation vector $\left(\delta_{\alpha}^{(k)}\right)_{\alpha \in \mathcal{A} \text {. }}$

The operator $S(k, l)$ maps $\Gamma_{s}^{(k)}$ onto $\Gamma_{s}^{(l)}$. Therefore we can define a quotient operator

$$
S_{b}(k, l): \Gamma^{(k)} / \Gamma_{s}^{(k)} \rightarrow \Gamma^{(l)} / \Gamma_{s}^{(l)} .
$$

As we have quotiented out the stable directions, it is not unreasonable to expect that the norm of the inverse of $S_{b}(k, l)$ is not too large. This is what our third condition is about:

(c) for any $\varepsilon>0$, there exists $C_{\varepsilon}>0$ such that, for all $l \geq k$, we have

$$
\begin{aligned}
\left\|\left[S_{b}(k, l)\right]^{-1}\right\| & \leq C_{\varepsilon}\|Q(l)\|^{\varepsilon}, \\
\left\|\left.S(k, l)\right|_{\Gamma_{s}^{(k)}}\right\| & \leq C_{\varepsilon}\|Q(l)\|^{\varepsilon} .
\end{aligned}
$$

Remark. The second estimate in (c) was wrongly omitted in MMY].

1.3.4. Roth-type interval exchange maps. We say that an i.e.m. $T$ is of Roth type if it satisfies Keane's condition and conditions (a), (b), (c).

In the next section, we will solve the cohomological equation for i.e.m.'s of Roth type. In Section 4 we will prove the following.

Theorem. Roth-type interval exchange maps form a subset of full measure.

We also observe that if an i.e.m. $T$ satisfies Keane's condition, and its RauzyVeech continued fraction is eventually periodic (meaning that the path $\gamma$ in the Rauzy diagram is eventually periodic), then conditions (ã), (b) and (c) are automatically satisfied and therefore $T$ is of Roth type. 


\section{The COHOMOLOGICAL EQUATION}

\subsection{The theorem of Gottschalk and Hedlund.}

2.1.1. The statement. We recall the following theorem of Gottschalk and Hedlund. Let $X$ be a compact topological space, $f$ a minimal homeomorphism of $X$ and $\psi$ a real-valued continuous function on $X$. Given $x_{0} \in X$ and $n \geq 1$ we denote by $S_{n} \psi\left(x_{0}\right)$ the Birkhoff sum $\sum_{j=0}^{n-1} \psi \circ T^{j}\left(x_{0}\right)$. Suppose that there exists a point $x_{0} \in X$ and a positive constant $C$ such that for all positive integers $n$ one has $\left|S_{n} \psi\left(x_{0}\right)\right| \leq C$. Then the cohomological equation

$$
\varphi \circ f-\varphi=\psi
$$

has a continuous solution $\varphi$.

2.1.2. Application to interval exchange maps. Let $T$ be an i.e.m. satisfying Keane's condition. Then $T$ is minimal but not continuous. However, the following wellknown construction, reminiscent of Denjoy counterexamples, allows us to bypass this problem.

For $n \geq 0$, define

$$
\begin{aligned}
& D_{0}(n)=\left\{T^{-n}\left(j_{0}(0, \alpha)\right), \alpha \in \mathcal{A}, \pi_{0}(\alpha)>1\right\}, \\
& D_{1}(n)=\left\{T^{+n}\left(j_{1}(0, \alpha)\right), \alpha \in \mathcal{A}, \pi_{1}(\alpha)>1\right\} .
\end{aligned}
$$

It follows from the Keane property that these sets are disjoint from each other and do not contain 0 .

Define an atomic measure $\mu$ by

$$
\mu=\sum_{n \geq 0} \sum_{y \in D_{0}(n) \sqcup D_{1}(n)} 2^{-n} \delta_{y}
$$

and the increasing maps $i^{+}, i^{-}: I \rightarrow \mathbb{R}$ by

$$
\begin{aligned}
& i^{-}(y)=y+\mu([0, y)), \\
& i^{+}(y)=y+\mu([0, y]) .
\end{aligned}
$$

We therefore have

$$
\begin{aligned}
& i^{+}(y)<i^{-}\left(y^{\prime}\right) \text { for } y<y^{\prime}, \\
& i^{+}(y)=i^{-}(y) \text { for } y \notin \bigsqcup_{n \geq 0}\left(D_{0}(n) \sqcup D_{1}(n)\right), \\
& i^{+}(y)=i^{-}(y)+2^{-n} \text { for } y \in D_{0}(n) \sqcup D_{1}(n) .
\end{aligned}
$$

We also define

$$
i^{-}\left(\lambda^{*}\right)=\lambda^{*}+4(d-1)=\lim _{y \nearrow \lambda^{*}} i^{ \pm}(y)
$$

and

$$
K=i^{-}(I) \cup i^{+}(I) \cup\left\{i^{-}\left(\lambda^{*}\right)\right\}=\overline{i^{-}(I)}=\overline{i^{+}(I)} .
$$

As $T$ is minimal, $K$ is a Cantor set whose gaps are the intervals $\left(i^{-}(y), i^{+}(y)\right)$, $y \in \bigcup_{n \geq 0} \bigcup_{\varepsilon \in\{0,1\}} D_{\varepsilon}(n)$.

Proposition. There is a unique continuous map $\hat{T}: K \rightarrow K$ such that $\hat{T} \circ i^{+}=$ $i^{+} \circ T$ on I. Moreover, $\hat{T}$ is a minimal homeomorphism. 
The elementary proof is left to the reader.

Let $\psi: I \rightarrow \mathbb{R}$ be a function which is continuous on each $j_{0}\left(I_{\alpha}\right)$, with finite limits at the right endpoints of each $j_{0}\left(I_{\alpha}\right)$. There is a unique continuous function $\hat{\psi}, K \rightarrow \mathbb{R}$ such that $\psi(y)=\hat{\psi} \circ i^{+}(y)$ for all $y \in I$. Assume that, for some $x_{0} \in I$ the Birkhoff sums of $\psi$ for $T$ are bounded. Then the same is true for the Birkhoff sums of $\hat{\psi}$ for $\hat{T}$ at the point $\hat{x}_{0}=i^{+}\left(x_{0}\right)$. By the theorem of Gottschalk and Hedlund, there is a continuous function $\hat{\varphi}, K \rightarrow \mathbb{R}$ satisfying $\hat{\psi}=\hat{\varphi} \circ \hat{T}-\hat{\varphi}$. Define, for $y \in I$,

$$
\varphi(y)=\hat{\varphi} \circ i^{+}(y)
$$

In general, $\varphi$ is not continuous. However it is bounded and satisfies $\varphi \circ T-\varphi=\psi$. In the following, we will show that under appropriate circumstances certain Birkhoff sums are bounded.

\subsection{Special Birkhoff sums.}

2.2.1. Let $T$ be an i.e.m. satisfying Keane's condition. Denote by $T^{(k)}$ the i.e.m. obtained by the accelerated Zorich algorithm (with $D=d-1$ in 1.2.4).

Let $\varphi: I^{(k)} \rightarrow \mathbb{R}$ be a function defined on the domain $I^{(k)}$ of $T^{(k)}$. Also let $l \geq k$. For $\beta \in \mathcal{A}, x \in j_{0}\left(I_{\beta}^{(l)}\right)$, the return time of $x$ into $I^{(l)}$ under iteration of $T^{(k)}$ is $Q_{\beta}(k, l)$. Define a function

$$
S(k, l) \varphi: I^{(l)} \rightarrow \mathbb{R}
$$

by the formula

$$
S(k, l)(\varphi)(x)=\sum_{0 \leq i<Q_{\beta}(k, l)} \varphi\left(\left(T^{(k)}\right)^{i}(x)\right),
$$

for $x \in j_{0}\left(I_{\beta}^{(l)}\right)$. Observe that when $\varphi$ is constant on each $j_{0}\left(I_{\beta}^{(k)}\right)$, the same is true of $S(k, l)(\varphi)$ in $I^{(l)}$ and the corresponding linear operator has ${ }^{t} Q(k, l)$ as matrix in the canonical basis, as anticipated in 1.3.2.

We just write $S(k)$ for $S(0, k)$.

2.2.2. Some elementary properties of the operators $S(k, l)$.

2.2.2.1. For $m \geq l \geq k$ one has

$$
S(k, m)=S(l, m) \circ S(k, l) .
$$

2.2.2.2. The operators $S(m, n)$ preserve all regularity classes which are invariant by restriction, sum and translation.

2.2.2.3. If $\varphi$ is an integrable function on $I^{(k)}$,

$$
\int_{I^{(k)}} \varphi(x) d x=\int_{I^{(l)}}(S(k, l) \varphi)(x) d x
$$

2.2.2.4. The operators $S(k, l)$ commute with taking derivatives.

2.2.2.5. If the restriction of $\varphi$ to each $j_{0}\left(I_{\alpha}^{(k)}\right)$ is a polynomial of degree $\leq \mu$, the restriction of $S(k, l) \varphi$ to each $j_{0}\left(I_{\beta}^{(l)}\right)$ is also a polynomial of degree $\leq \mu$. The case $\mu=0$ has already been considered.

2.2.2.6. Denote by BV $\left(\bigsqcup I_{\alpha}^{(k)}\right)$ the space of functions $\varphi$ on $I^{(k)}$ whose restriction to each $j_{0}\left(I_{\alpha}^{(k)}\right)$ has bounded variation and define

$$
\operatorname{Var} \varphi=\left.\sum_{\alpha} \operatorname{Var} \varphi\right|_{j_{0}\left(I_{\alpha}^{(k)}\right)} .
$$


(We do not take into account the discontinuities of $\varphi$ at the discontinuity points of $T^{(k)}$.) Then $S(k, l)$ sends BV $\left(\bigsqcup I_{\alpha}^{(k)}\right)$ into BV $\left(\bigsqcup I_{\alpha}^{(l)}\right)$ and we have

$$
\operatorname{Var} S(k, l) \varphi \leq \operatorname{Var} \varphi \text {. }
$$

2.2.3. Reduction of Birkhoff sums to special Birkhoff sums. For diffeomorphisms of the circle with irrational rotation number, when trying to estimate the Birkhoff sums of some function, it is a standard trick to consider first the sums associated to the denominators of the convergents of the rotation number. We will do the same here.

Let $\varphi: I \rightarrow \mathbb{R}$ be a function, $x \in I$, and $N>0$. We want to compute the Birkhoff sums

$$
S_{N} \varphi(x)=\sum_{i=0}^{N-1} \varphi\left(T^{i}(x)\right)
$$

(with $\left.T=T^{(0)}\right)$.

We first replace $x$ by the point in the orbit $\left\{x, T(x), \ldots, T^{N-1}(x)\right\}$ which is closest to 0 and cut the Birkhoff sum into two parts (one for $T$ and the other for $T^{-1}$ ). Let us assume to keep notation simple that $x$ is actually closest to the origin.

Let $k \geq 0$ be the largest integer such that at least one of the points $T(x), \ldots$, $T^{N-1}(x)$ belongs to $I^{(k)}$; because $T^{(k)}$ is the first return map into $I^{(k)}$, these points are precisely $T^{(k)}(x),\left(T^{(k)}\right)^{2}(x), \ldots,\left(T^{(k)}\right)^{b(k)}(x)$ for some integer $b(k)>0$. Moreover, as none of these points belongs to $I^{(k+1)}$ we must have

$$
b(k)<\operatorname{Max}_{\beta \in \mathcal{A}} \sum_{\alpha \in \mathcal{A}} Z_{\alpha \beta}(k+1),
$$

the right-hand term being the largest return time of $T^{(k)}$ into $I^{(k+1)}$.

We set $x_{k}=x, x_{k-1}=\left(T^{(k)}\right)^{b(k)}(x)$ and define inductively $b(l)$ and $x_{l}$ for $0 \leq l \leq k$.

The point $x_{l}$ has the property that it belongs to $I^{(l)}$ and none of the points $T\left(x_{l}\right), \ldots, T^{N}(x)$ belongs to $I^{(l+1)}$. Those that belong to $I^{(l)}$ are $T^{(l)}\left(x_{l}\right), \ldots$, $\left(T^{(l)}\right)^{b(l)}\left(x_{l}\right)$ for some integer $b(l) \geq 0$. We have

$$
b(l)<\operatorname{Max}_{\beta \in \mathcal{A}} \sum_{\alpha \in \mathcal{A}} Z_{\alpha \beta}(l+1),
$$

We define $x_{l-1}=\left(T^{(l)}\right)^{b(l)}\left(x_{l}\right)$. The process stops when $x_{l}=T^{N}(x)($ or $l=0)$. From this construction it is obvious that we have

$$
S_{N} \varphi(x)=\sum_{l=0}^{k} \sum_{0 \leq i<b(l)} S(l) \varphi\left(\left(T^{(l)}\right)^{i}\left(x_{l}\right)\right),
$$

which in particular implies, if $\varphi$ is bounded:

$$
\left|S_{N} \varphi(x)\right| \leq \sum_{l=0}^{k}\|Z(l+1)\|\|S(l) \varphi\|_{L^{\infty}},
$$

where $\|Z(l+1)\|=\operatorname{Max}_{\beta \in \mathcal{A}} \sum_{\alpha \in \mathcal{A}} Z_{\alpha \beta}(l+1)$.

In particular, if we are able to show that for some $\omega>0$ we have

$$
\|S(l) \varphi\|_{L^{\infty}} \leq C\|Q(l)\|^{-\omega}\|\varphi\|,
$$

and condition (a) in 1.3.1 is satisfied, then the Birkhoff sums of $\varphi$ will be bounded. 


\subsection{Estimates for functions of bounded variation.}

2.3.1. Denote by $\mathrm{BV}_{*}\left(\bigsqcup I_{\alpha}^{(k)}\right)$ the subspace of $\mathrm{BV}\left(\bigsqcup I_{\alpha}^{(k)}\right)$ formed by the functions of mean 0 . The operator $S(k, l)$ sends this subspace into $\mathrm{BV}_{*}\left(\bigsqcup I_{\alpha}^{(l)}\right)$.

Let $\varphi \in \mathrm{BV}_{*}\left(\bigsqcup I_{\alpha}^{(k)}\right)$. We write

$$
S(k, k+1)(\varphi)=\varphi_{k+1}+\chi_{k+1},
$$

with $\chi_{k+1} \in \Gamma_{*}^{(k+1)}$ and $\varphi_{k+1}$ of mean zero on $e a c h j_{0}\left(I_{\alpha}^{(k+1)}\right)$. Then we go on with

$$
S(j, j+1)\left(\varphi_{j}\right)=\varphi_{j+1}+\chi_{j+1}
$$

with $\chi_{j+1} \in \Gamma_{*}^{(j+1)}$ and $\varphi_{j+1}$ of mean zero on each $j_{0}\left(I_{\alpha}^{(j+1)}\right)$. We obtain, for $l>k$,

$$
S(k, l) \varphi=S(l-1, l) \varphi_{l-1}+\sum_{k<j<l} S(j, l) \chi_{j} .
$$

2.3.2. As $\varphi_{j}$ differs from $S(k, j) \varphi$ by a function in $\Gamma_{*}^{(j)}$ and has mean zero on each $j_{0}\left(I_{\alpha}^{(j)}\right)$ we have (see 2.2.2.6)

$$
\left\|\varphi_{j}\right\|_{L^{\infty}} \leq \operatorname{Var} \varphi_{j} \leq \operatorname{Var} \varphi .
$$

On the other hand, we have

$$
\begin{aligned}
\left\|\chi_{j}\right\|_{L^{\infty}} & \leq\left\|S(j-1, j) \varphi_{j-1}\right\|_{L^{\infty}} \\
& \leq\|Z(j)\|\left\|\varphi_{j-1}\right\|_{L^{\infty}}
\end{aligned}
$$

(with $\varphi_{j-1}=\varphi$ when $j=k+1$ ). We obtain therefore

$$
\begin{aligned}
\|S(k, l) \varphi\|_{L^{\infty}} & \leq\|Z(k+1)\|\left\|\left.S(k+1, l)\right|_{\Gamma_{*}^{(k+1)}}\right\|\left\|\varphi_{0}\right\|_{L^{\infty}} \\
& +\sum_{k<j<l}\|Z(j+1)\|\left\|\left.S(j+1, l)\right|_{\Gamma_{*}^{(j+1)}}\right\| \operatorname{Var} \varphi .
\end{aligned}
$$

2.3.3. We now take $k=0$ and estimate the sum

$$
\sum_{0 \leq j<l}\|Z(j+1)\|\left\|\left.S(j+1, l)\right|_{\Gamma_{*}^{(j+1)}}\right\|
$$

assuming that conditions (a), (b) of Section 1.3 are satisfied.

On one side we have, by condition (a), for all $\varepsilon>0$ :

$$
\|Z(j+1)\| \leq C_{\varepsilon}\|Q(j)\|^{\varepsilon} .
$$

To estimate $\left\|\left.S(j+1, l)\right|_{\Gamma_{*}^{(j+1)}}\right\|$ we distinguish two cases. We assume condition (b) of 1.3.2, which involves an exponent $1-\theta$ with $\theta>0$.

i) Assume first that $\|Q(j+1)\| \leq\|Q(l)\|^{\theta / d}$. As $Q(j+1)$ belongs to $\operatorname{SL}(d, \mathbb{Z})$, we have

$$
\left\|(Q(j+1))^{-1}\right\| \leq c\|Q(l)\|^{\theta \frac{d-1}{d}} .
$$

Next we write

$$
S(j+1, l)=S(0, l) \circ(S(0, j+1))^{-1},
$$

and it follows from condition (b) that we have

$$
\left\|\left.S(j+1, l)\right|_{\Gamma_{*}^{(j+1)}}\right\| \leq C\|Q(l)\|^{1-\frac{\theta}{d}} .
$$


ii) Assume now that $\|Q(j+1)\|>\|Q(l)\|^{\theta / d}$. If $l \leq j+2 d-2(l \leq j+3$ when $d=2$ ), we just use

$$
\left\|\left.S(j+1, l)\right|_{\Gamma_{*}^{(j+1)}}\right\| \leq C_{\varepsilon}\|Q(l)\|^{\varepsilon}
$$

by condition (a). If $l>j+2 d-2(l>j+3)$, we write

$$
S(0, l)=S\left(j^{\prime}, l\right) S\left(j+1, j^{\prime}\right) S(0, j+1)
$$

with $j^{\prime}=j+2 d-2\left(j^{\prime}=j+3\right.$ when $\left.d=2\right)$. As the entries of $Q\left(j+1, j^{\prime}\right)$ are positive integers we have

$$
\|Q(0, l)\| \geq C\left\|Q\left(j^{\prime}, l\right)\right\|\|Q(0, j+1)\|,
$$

which implies

$$
\left\|Q\left(j^{\prime}, l\right)\right\| \leq C\|Q(l)\|^{1-\frac{\theta}{d}} .
$$

As we have also

$$
\left\|Q\left(j+1, j^{\prime}\right)\right\| \leq C_{\varepsilon}\left\|Q\left(j^{\prime}\right)\right\|^{\varepsilon},
$$

we obtain in this case that

$$
\left\|\left.S(j+1, l)\right|_{\Gamma^{(j+1)}}\right\| \leq C_{\varepsilon}\|Q(l)\|^{1-\frac{\theta}{d}+\varepsilon} .
$$

Putting the two cases together and inserting this in the sum, we obtain

Proposition. For $\varphi \in B V_{*}\left(\bigsqcup_{\alpha \in \mathcal{A}} I_{\alpha}^{(0)}\right), l \geq 0$, one has

$$
\|S(l) \varphi\|_{L^{\infty}} \leq C\|Q(l)\|^{1-\frac{\theta}{2 d}}\|\varphi\|_{B V} .
$$

Remark. In case i), the estimate we got for $Q(j+1)^{-1}$ is far from optimal (it should be of the order of $Q(j+1)$ ) but sufficient for our purposes.

\subsection{Primitives of functions of bounded variation.}

2.4.1. For $k \geq 0$, we will denote by $\mathrm{BV}^{1}\left(\bigsqcup_{\alpha \in \mathcal{A}} I_{\alpha}^{(k)}\right)$ the space of functions $\varphi$ : $I^{(k)} \rightarrow \mathbb{R}$ which are absolutely continuous on each $\left.j_{0}\left(I_{\alpha}^{(k)}\right)\right)$ and whose derivative on each $j_{0}\left(I_{\alpha}^{(k)}\right)$ ) is of bounded variation. The condition that the mean value of the derivative is zero defines a hyperspace $\mathrm{BV}_{*}^{1}\left(\bigsqcup_{\alpha \in \mathcal{A}} I_{\alpha}^{(k)}\right)$. We recall from 1.3.3 the subspace $\Gamma_{s}^{(k)}$ of $\Gamma^{(k)}$. We will denote by $\overline{\mathrm{BV}}_{*}^{1}\left(\bigsqcup_{\alpha \in \mathcal{A}} I_{\alpha}^{(k)}\right)$ the quotient of $\mathrm{BV}_{*}^{1}\left(\bigsqcup_{\alpha \in \mathcal{A}} I_{\alpha}^{(k)}\right)$ by this finite-dimensional subspace.

Given $\varphi \in \mathrm{BV}_{*}\left(\bigsqcup_{\alpha \in \mathcal{A}} I_{\alpha}^{(0)}\right)$, we will find a primitive $\Phi$ of $\varphi$ (given a priori by $d$ constants of integration, one for each $I_{\alpha}^{(0)}$ ) for which the special Birkhoff sums are small. The primitive $\Phi$ will actually be uniquely determined $\bmod \Gamma_{s}^{(0)}$, i.e. in $\overline{\mathrm{BV}}_{*}^{1}\left(\bigsqcup_{\alpha \in \mathcal{A}} I_{\alpha}^{(0)}\right)$.

2.4.2. For any $\varphi \in \mathrm{BV}_{*}\left(\bigsqcup_{\alpha \in \mathcal{A}} I_{\alpha}^{(k)}\right)$, denote by $P_{0}^{(k)} \varphi$ the class in $\overline{\mathrm{BV}}_{*}^{1}\left(\bigsqcup_{\alpha \in \mathcal{A}} I_{\alpha}^{(k)}\right)$ of the primitive of $\varphi$ which has mean zero on each $j_{0}\left(I_{\alpha}^{(k)}\right)$.

This is the most natural choice of primitive, but unfortunately the special Birkhoff sums $S(k, l)$ do not commute with these primitive operators, i.e. they do not preserve the condition of being of mean value 0 on each $j_{0}\left(I_{\alpha}^{(k)}\right)$.

Therefore, we will modify $P_{0}^{(k)}$, considering

$$
P^{(k)}=P_{0}^{(k)}+\Delta P^{(k)},
$$


where

$$
\Delta P^{(k)}: \mathrm{BV}_{*}\left(\bigsqcup_{\alpha \in \mathcal{A}} I_{\alpha}^{(k)}\right) \rightarrow \Gamma^{(k)} / \Gamma_{s}^{(k)}
$$

is a bounded linear operator. We want this new choice to be equivariant:

$$
S(k, l) \circ P^{(k)}=P^{(l)} \circ S(k, l) .
$$

This leads to the following equation for $\Delta P^{(k)}$. Define

$$
\Lambda(k, l)=P_{0}^{(l)} \circ S(k, l)-S(k, l) \circ P_{0}^{(k)} .
$$

This is a bounded linear map from $\mathrm{BV}_{*}\left(\bigsqcup_{\alpha \in \mathcal{A}} I_{\alpha}^{(k)}\right)$ to $\Gamma^{(l)} / \Gamma_{s}^{(l)}$. Then we should have

$$
S_{b}(k, l) \circ \Delta P^{(k)}-\Delta P^{(l)} \circ S(k, l)=\Lambda(k, l),
$$

where $S_{b}$ was defined in 1.3.3.

Equation $(*)$ has the formal solution

$$
\Delta P^{(k)}=\sum_{l>k}\left(S_{b}(k, l)\right)^{-1} \circ \Lambda(l-1, l) \circ S(k, l-1),
$$

and we will check next that this defines indeed the required primitive.

2.4.3. Estimate for $\Lambda(l-1, l)$. Let $\varphi \in \mathrm{BV}_{*}\left(\bigsqcup_{\alpha \in \mathcal{A}} I_{\alpha}^{(l-1)}\right)$. As $P_{0}^{(l-1)} \varphi$ has mean zero on each $j_{0}\left(I_{\alpha}^{(l-1)}\right)$, we have

$$
\left\|P_{0}^{(l-1)} \varphi\right\|_{L^{\infty}} \leq\left(\operatorname{Max}_{\alpha \in \mathcal{A}} \lambda_{\alpha}^{(l-1)}\right)\|\varphi\|_{L^{\infty}} .
$$

On the other hand we have

$$
\begin{gathered}
\|S(l-1, l) \varphi\|_{L^{\infty}} \leq\|Z(l)\|\|\varphi\|_{L^{\infty}}, \\
\left\|S(l-1, l) P_{0}^{(l-1)} \varphi\right\|_{L^{\infty}} \leq\|Z(l)\|\left\|P_{0}^{(l-1)} \varphi\right\|_{L^{\infty}} .
\end{gathered}
$$

Finally, we get

$$
\left\|P_{0}^{(l)} S(l-1, l) \varphi\right\|_{L^{\infty}} \leq\left(\operatorname{Max}_{\alpha \in \mathcal{A}} \lambda_{\alpha}^{(l)}\right)\|Z(l)\|\|\varphi\|_{L^{\infty}},
$$

which allows us to conclude that

$$
\|\Lambda(l-1, l) \varphi\|_{L^{\infty}} \leq 2\|Z(l)\|\left(\operatorname{Max}_{\alpha \in \mathcal{A}} \lambda_{\alpha}^{(l-1)}\right)\|\varphi\|_{L^{\infty}} .
$$

2.4.4. Assume now the three conditions (a), (b) and (c) of 1.3. From 1.3.1, we get

$$
\begin{aligned}
\operatorname{Max}_{\alpha \in \mathcal{A}} \lambda_{\alpha}^{(l-1)} & \leq C_{\varepsilon}\|Q(l-1)\|^{\varepsilon-1}, \\
\|Z(l)\| & \leq C_{\varepsilon}\|Q(l-1)\|^{\varepsilon},
\end{aligned}
$$

and from condition (c) that

$$
\left\|\left(S_{b}(0, l)\right)^{-1}\right\| \leq C_{\varepsilon}\|Q(l)\|^{\varepsilon} .
$$

On the other hand, from the Proposition in 2.3, we obtain

$$
\|S(0, l-1) \varphi\|_{L^{\infty}} \leq C\|Q(l-1)\|^{1-\theta / 2 d}\|\varphi\|_{B V} .
$$

Therefore, for $k=0$, the series $(* *)$ in 2.4 .2 is converging and we obtain

$$
\left\|\Delta P^{(0)} \varphi\right\| \leq\left(\sum_{l>0} C_{\varepsilon}^{\prime}\|Q(l)\|^{3 \varepsilon-\theta / 2 d}\right)\|\varphi\|_{B V} .
$$

Indeed, we take $\varepsilon<\theta / 6 d$ and observe that it follows from the Lemma in 1.2.4 that $\|Q(l)\|$ grows at least exponentially fast. In the same way, as $T^{(k)}$ satisfies 
also conditions (a), (b), (c) (with worse constants but the same exponent $\theta$ ), the series $(* *)$ will converge for all $k \geq 0$. In this case, we prefer to estimate directly $\Delta P^{(k)} S(0, k) \varphi$ for $\varphi \in \mathrm{BV}_{*}\left(\bigsqcup_{\alpha \in \mathcal{A}} I_{\alpha}^{(0)}\right)$. We have

$$
\left\|\Delta P^{(k)} S(0, k) \varphi\right\| \leq \sum_{l>k}\left\|\left(S_{b}(k, l)\right)^{-1}\right\|\|\Lambda(l-1, l)\|\|S(0, l-1) \varphi\|_{L^{\infty}} .
$$

The above estimates now give

$$
\begin{aligned}
\left\|\Delta P^{(k)} S(0, k) \varphi\right\| & \leq\left(\sum_{l>k} C_{\varepsilon}^{\prime}\|Q(l)\|^{3 \varepsilon-\theta / 2 d}\right)\|\varphi\|_{B V} \\
& \leq C\|Q(k)\|^{-\theta / 3 d}\|\varphi\|_{B V} .
\end{aligned}
$$

2.4.5. Special Birkhoff sums for $P^{(0)} \varphi$. Let $\varphi \in \mathrm{BV}_{*}\left(\bigsqcup_{\alpha \in \mathcal{A}} I_{\alpha}^{(0)}\right)$ and $\Phi \in$ $\mathrm{BV}_{*}^{1}\left(\bigsqcup_{\alpha \in \mathcal{A}} I_{\alpha}^{(0)}\right)$ such that the class $\bmod \Gamma_{s}^{(0)}$ of $\Phi$ is $P^{(0)} \varphi$. The class $\bmod \Gamma_{s}^{(k)}$ of $S(k) \Phi$ is $P^{(k)} S(k) \varphi$ by construction.

From the definition of $P_{0}^{(k)}$ and 2.3 , we have

$$
\left\|P_{0}^{(k)} S(k) \varphi\right\|_{L^{\infty}} \leq C\left(\operatorname{Max}_{\alpha \in \mathcal{A}} \lambda_{\alpha}^{(k)}\right)\|Q(k)\|^{1-\theta / 2 d}\|\varphi\|_{B V},
$$

with $\operatorname{Max}_{\alpha \in \mathcal{A}} \lambda_{\alpha}^{(k)} \leq C_{\varepsilon}\|Q(k)\|^{\varepsilon-1}$ by condition (a). Joining this with the estimate for $\Delta P^{(k)}$ above, we obtain

$$
\begin{aligned}
\left\|S(k) P^{(0)} \varphi\right\| & =\left\|P^{(k)} S(k) \varphi\right\| \\
& \leq C\|Q(k)\|^{-\theta / 3 d}\|\varphi\|_{B V} .
\end{aligned}
$$

By the definition of a quotient norm, this means that we may write in $\mathrm{BV}_{*}^{1}\left(\bigsqcup_{\alpha \in \mathcal{A}} I_{\alpha}^{(k)}\right)$ :

$$
S(k) \Phi=\Phi_{k}+\chi_{k},
$$

with $\chi_{k} \in \Gamma_{s}^{(k)}$ and

$$
\left\|\Phi_{k}\right\|_{L^{\infty}} \leq C\|Q(k)\|^{-\theta / 3 d}\|\varphi\|_{B V} .
$$

We have then

$$
\begin{aligned}
\chi_{k+1} & =S(k, k+1) \chi_{k}+S(k, k+1) \Phi_{k}-\Phi_{k+1} \\
& :=S(k, k+1) \chi_{k}+\Delta \chi_{k+1},
\end{aligned}
$$

with $\left\|\Delta \chi_{k+1}\right\| \leq C\|Q(k+1)\|^{-\theta / 4 d}\|\varphi\|_{B V}$ (using once more condition (a)). Then

$$
S(k) \Phi=\Phi_{k}+\sum_{j \leq k} S(j, k) \Delta \chi_{j} .
$$

In the sum, we separate two cases. Recall that there exists $\sigma>0, C>0$, such that

$$
\left\|\left.S(j)\right|_{\Gamma_{s}^{(0)}}\right\| \leq C\|Q(j)\|^{-\sigma},
$$

for all $j \geq 0$. If $\|Q(j)\| \leq\|Q(k)\|^{\sigma / d}$, we write $S(j, k)=S(k) \circ(S(j))^{-1}$ and get

$$
\begin{aligned}
\left\|S(j, k) \Delta \chi_{j}\right\| & \leq C\|Q(k)\|^{-\sigma}\left\|(S(j))^{-1} \Delta \chi_{j}\right\| \\
& \leq C\|Q(k)\|^{-\sigma}\|Q(j)\|^{d-1}\left\|\Delta \chi_{j}\right\| \\
& \leq C\|Q(k)\|^{-\sigma / d}\|\varphi\|_{B V} .
\end{aligned}
$$


In case $\|Q(j)\|>\|Q(k)\|^{\sigma / d}$, we use the second estimate in condition (c) to get

$$
\begin{aligned}
\left\|S(j, k) \Delta \chi_{j}\right\| & \leq C_{\varepsilon}\|Q(k)\|^{\varepsilon}\left\|\Delta \chi_{j}\right\| \\
& \leq C_{\varepsilon}\|Q(k)\|^{\varepsilon-\theta \sigma / 4 d^{2}}\|\varphi\|_{B V} .
\end{aligned}
$$

We have thus proved the

Theorem. Let $T$ be an i.e.m. of Roth type. There exists $\omega>0$, depending only on $\sigma$ and $\theta$ in (b), (c), such that the special Birkhoff sums $S(k) \Phi$ satisfy

$$
\|S(k) \Phi\|_{L^{\infty}} \leq C\|Q(k)\|^{-\omega}\|\varphi\|_{B V} .
$$

Corollary. Let $T$ be an i.e.m. of Roth type, $\varphi \in B V_{*}\left(\bigsqcup_{\alpha \in \mathcal{A}} I_{\alpha}^{(0)}\right)$. For any primitive $\Phi$ of $\varphi$ whose class lies in $P^{(0)} \varphi$, we can solve the cohomological equation

$$
\Psi \circ T-\Psi=\Phi
$$

with a bounded solution $\Psi$.

Proof. This follows from the theorem, taking into account the remarks at the end of Sections 2.1 and 2.2.3.

\section{Suspensions of InTERVAL EXCHANGE MAPS}

We first recall, basically to fix notation, how to suspend i.e.m.'s in order to get a Riemann surface with a holomorphic 1-form. The basic reference is V1].

3.1. Suspension data. Let $\left(\mathcal{A}, \pi_{0}, \pi_{1}\right)$ be admissible combinatorial data, and let $T$ be an i.e.m. of this combinatorial type, determined by length data $\left(\lambda_{\alpha}\right)_{\alpha \in \mathcal{A}}$. We will construct a Riemann surface with a flow which can be considered as a suspension of $T$. In order to do this, we need data which we call suspension data. We will identify $\mathbb{R}^{2}$ with $\mathbb{C}$. Consider a family $\tau=\left(\tau_{\alpha}\right)_{\alpha \in \mathcal{A}} \in \mathbb{R}^{\mathcal{A}}$. To this family we associate

$$
\begin{aligned}
& \zeta_{\alpha}=\lambda_{\alpha}+i \tau_{\alpha}, \quad \alpha \in \mathcal{A}, \\
& \xi_{\alpha}^{\varepsilon}=\sum_{\pi_{\varepsilon} \beta \leq \pi_{\varepsilon} \alpha} \zeta_{\beta}, \quad \alpha \in \mathcal{A}, \quad \varepsilon \in\{0,1\} .
\end{aligned}
$$

We always have $\xi_{\alpha_{0}}^{0}=\xi_{\alpha_{1}}^{1}$, where as before $\pi_{\varepsilon}\left(\alpha_{\varepsilon}\right)=d$. We say that $\tau$ defines suspension data if the following inequalities hold:

$$
\begin{aligned}
& \Im m \xi_{\alpha}^{0}>0 \text { for all } \alpha \in \mathcal{A}, \alpha \neq \alpha_{0}, \\
& \Im m \xi_{\alpha}^{1}<0 \text { for all } \alpha \in \mathcal{A}, \alpha \neq \alpha_{1} .
\end{aligned}
$$

We also set

$$
\theta_{\alpha}=\xi_{\alpha}^{1}-\xi_{\alpha}^{0}, \alpha \in \mathcal{A}
$$

We then have

$$
\begin{aligned}
\theta & =\Omega \zeta, \\
\Re e \theta & =\delta,
\end{aligned}
$$

and define

$$
h=-\Im m \theta=-\Omega \tau \text {. }
$$

One has $h_{\alpha}>0$ for all $\alpha \in \mathcal{A}$, because of the formula

$$
\theta_{\alpha}=\left(\xi_{\alpha}^{1}-\zeta_{\alpha}\right)-\left(\xi_{\alpha}^{0}-\zeta_{\alpha}\right) .
$$


One has also

$$
\Im m \xi_{\alpha_{0}}^{0}=\Im m \xi_{\alpha_{1}}^{1} \in\left[-h_{\alpha_{1}}, h_{\alpha_{0}}\right] .
$$

3.2. Construction of a Riemann surface. Let $\left(\mathcal{A}, \pi_{0}, \pi_{1}\right)$ and $\left(\zeta_{\alpha}=\lambda_{\alpha}+\right.$ $\left.i \tau_{\alpha}\right)_{\alpha \in \mathcal{A}}$ be as above. For $\alpha \in \mathcal{A}$, consider the rectangles in $\mathbb{C}=\mathbb{R}^{2}$ :

$$
\begin{aligned}
& R_{\alpha}^{0}=\left(\Re e \xi_{\alpha}^{0}-\lambda_{\alpha}, \Re e \xi_{\alpha}^{0}\right) \times\left[0, h_{\alpha}\right], \\
& R_{\alpha}^{1}=\left(\Re e \xi_{\alpha}^{1}-\lambda_{\alpha}, \Re e \xi_{\alpha}^{1}\right) \times\left[-h_{\alpha}, 0\right],
\end{aligned}
$$

and the segments

$$
\begin{aligned}
& S_{\alpha}^{0}=\left\{\Re e \xi_{\alpha}^{0}\right\} \times\left[0, \Im m \xi_{\alpha}^{0}\right), \alpha \neq \alpha_{0}, \\
& S_{\alpha}^{1}=\left\{\Re e \xi_{\alpha}^{1}\right\} \times\left(\Im m \xi_{\alpha}^{1}, 0\right], \alpha \neq \alpha_{1} .
\end{aligned}
$$

Also let $S_{\alpha_{0}}^{0}=S_{\alpha_{1}}^{1}$ be the half-open vertical segment $\left[\lambda^{*}, \xi_{\alpha_{0}}^{0}\right)=\left[\lambda^{*}, \xi_{\alpha_{1}}^{1}\right)$.

Define then

$$
R_{\zeta}=\bigcup_{\varepsilon \in\{0,1\}} \bigcup_{\alpha \in \mathcal{A}} R_{\alpha}^{\varepsilon} \bigcup_{\varepsilon \in\{0,1\}} \bigcup_{\alpha \in \mathcal{A}} S_{\alpha}^{\varepsilon} .
$$

The translation by $\theta_{\alpha}$ sends $R_{\alpha}^{0}$ onto $R_{\alpha}^{1}$. If $\xi_{\alpha_{0}}^{0}=\xi_{\alpha_{1}}^{1}=0, S_{\alpha_{0}}^{0}=S_{\alpha_{1}}^{1}$ is empty, $\xi_{\alpha_{1}}^{0}$ is the top right corner of $R_{\alpha_{1}}^{0}$ and $\xi_{\alpha_{0}}^{1}$ is the bottom right corner of $R_{\alpha_{0}}^{1}$. If $\xi_{\alpha_{0}}^{0}=$ $\xi_{\alpha_{1}}^{1}>0$, the translation by $\theta_{\alpha_{1}}$ sends the top part $\tilde{S}_{\alpha_{1}}^{0}=\left\{\Re e \xi_{\alpha_{1}}^{0}\right\} \times\left[h_{\alpha_{1}}, \Im m \xi_{\alpha_{1}}^{0}\right)$ of $S_{\alpha_{1}}^{0}$ onto $S_{\alpha_{1}}^{1}$. If $\xi_{\alpha_{0}}^{0}=\xi_{\alpha_{1}}^{1}<0$, the translation by $\theta_{\alpha_{0}}$ sends $S_{\alpha_{0}}^{0}$ onto the bottom part $\tilde{S}_{\alpha_{0}}^{1}=\left\{\Re e \xi_{\alpha_{0}}^{1}\right\} \times\left(\Im m \xi_{\alpha_{0}}^{1},-h_{\alpha_{0}}\right]$ of $S_{\alpha_{0}}^{1}$.

We use these translations to identify in $R_{\zeta}$ each $R_{\alpha}^{0}$ to each $R_{\alpha}^{1}$, and $S_{\alpha_{0}}^{0}=S_{\alpha_{1}}^{1}$ (if nonempty) to either $\tilde{S}_{\alpha_{1}}^{0}$ or $\tilde{S}_{\alpha_{0}}^{1}$.

Denote by $M_{\zeta}^{*}$ the topological space obtained from $R_{\zeta}$ by these identifications.

Observe that $M_{\zeta}^{*}$ inherits from $\mathbb{C}$ the structure of a Riemann surface, and also a nowhere-vanishing holomorphic 1 -form $\omega$ (given by $d z$ ) and a vertical vector field (given by $\frac{\partial}{\partial y}$ ).

3.3. Compactification of $M_{\zeta}^{*}$. Let $\overline{\mathcal{A}}$ be the set with $2 d-2$ elements of pairs $(\alpha, L)$ and $(\alpha, R)$, except that we identify $\left(\alpha_{0}, R\right)=\left(\alpha_{1}, R\right)$ and $\left(\alpha_{0}^{\prime}, L\right)=\left(\alpha_{1}^{\prime}, L\right)$, where $\pi_{\varepsilon}\left(\alpha_{\varepsilon}\right)=d, \pi_{\varepsilon}\left(\alpha_{\varepsilon}^{\prime}\right)=1$.

Let $\sigma$ be the permutation of $\overline{\mathcal{A}}$ defined by

$$
\begin{aligned}
& \sigma(\alpha, R)=\left(\beta_{0}, L\right), \\
& \sigma(\alpha, L)=\left(\beta_{1}, R\right),
\end{aligned}
$$

with $\pi_{0}\left(\beta_{0}\right)=\pi_{0}(\alpha)+1, \pi_{1}\left(\beta_{1}\right)=\pi_{1}(\alpha)-1$; in particular, we have

$$
\begin{aligned}
& \sigma\left(\alpha_{0}, R\right)=\left(\pi_{0}^{-1}\left(\pi_{0}\left(\alpha_{1}\right)+1\right), L\right), \\
& \sigma\left(\alpha_{1}^{\prime}, L\right)=\left(\pi_{1}^{-1}\left(\pi_{1}\left(\alpha_{0}^{\prime}\right)-1\right), R\right) .
\end{aligned}
$$

The permutation describes which half planes are met when one winds around an end of $M_{\zeta}^{*}$. Denote by $\Sigma$ the set of cycles of $\sigma$. To each $C \in \Sigma$ is associated in a one-to-one correspondance an end $q_{C}$ of $M_{\zeta}^{*}$. From the local structure around $q_{C}$, it is clear that the compactification $M_{\zeta}=M_{\zeta}^{*} \bigcup_{C \in \Sigma}\left\{q_{C}\right\}$ will be a compact Riemann surface, with the set of marked points $\bigcup_{C \in \Sigma}\left\{q_{C}\right\}=M_{\zeta} \backslash M_{\zeta}^{*}$ in canonical correspondance with $\Sigma$. Moreover, the 1-form $\omega$ extends to a holomorphic 1-form on $M_{\zeta}$; the length of a cycle $C$ is an even number $2 n_{C}$; the corresponding marked point $q_{C}$ is a zero of $\omega$ of order $n_{C}-1$. 
Let $\nu=\operatorname{card} \Sigma$, and let $g$ be the genus of $M_{\zeta}$. We have

$$
\begin{aligned}
d-1 & =\sum_{C \in \Sigma} n_{C}, \\
2 g-2 & =\sum_{C \in \Sigma}\left(n_{C}-1\right) ;
\end{aligned}
$$

hence

$$
d=2 g+\nu-1
$$

Example. Suppose that $\pi_{0}, \pi_{1}$ satisfy

$$
\pi_{0}(\alpha)+\pi_{1}(\alpha)=d+1, \text { for all } \alpha \in \mathcal{A} .
$$

If $d$ is even, there is only one cycle; we have $d=2 g$ and the only zero of $\omega$ has order $2 g-2$. If $d$ is odd, there are two cycles of equal length $d-1$; we have $d=2 g+1$, and each of the two zeros of $\omega$ has order $g-1$.

The vertical vector field on $M_{\zeta}^{*}$ does not extend (continuously) to $M_{\zeta}$ when $g>1$, unless one slows it near the marked points (which we will not do here). Nevertheless, it can be considered as a suspension of $T$ : starting from a point $(x, 0)$ on the bottom side of $R_{\alpha}^{0}$, one flows up until reaching the top side where the point $\left(x, h_{\alpha}\right)$ is identified with the point $\left(x+\delta_{\alpha}, 0\right)=(T(x), 0)$ in the top side of $R_{\alpha}^{1}$. The return time is $h_{\alpha}$. The vector field is not complete, as some orbits reach marked points in finite time.

\subsection{The cohomological equation for higher smoothness.}

3.4.1. In this section, we will relate the (discrete) cohomological equation for i.e.m.'s to the (continuous) cohomological equation for the vertical vector field on $M_{\zeta}$; this equation is

$$
\tilde{\Phi}=\frac{\partial}{\partial y} \tilde{\Psi}
$$

where now $\tilde{\Phi}, \tilde{\Psi}$ are functions on $M_{\zeta}$. This allows us to compare our results with the pioneering work of Forni ([Fo1] $)$. We will always assume, as he does, that $\tilde{\Phi}$ vanishes in the neighborhood of the marked points of $M_{\zeta}$.

Considering the cohomological equation on the surface leads naturally to some regularity assumptions on the interval. Because the datum $\Phi$ and the solution $\Psi$ are not related to the corresponding functions $\tilde{\Phi}, \tilde{\Psi}$ on the surface in the same way ( $\Psi$ is a restriction of $\tilde{\Psi}$ to a segment, while $\Phi$ is an integral), the regularity that we introduce for $\Phi$ and $\Psi$ are not of the same kind (even taking the loss of derivatives into account).

3.4.2. For each integer $r \geq 1$, we introduce the space $\mathrm{BV}_{*}^{r}(I)$ of functions $\Phi: I \rightarrow$ $\mathbb{R}$ such that

- for each $\alpha \in \mathcal{A}, \Phi$ is of class $\mathcal{C}^{r-1}$ on $j_{0}\left(I_{\alpha}\right), D^{r-1} \Phi$ is absolutely continuous on $j_{0}\left(I_{\alpha}\right)$ and $D^{r} \Phi$ is of bounded variation on $j_{0}\left(I_{\alpha}\right)$;

- each function $D^{l} \Phi$, for $0<l \leq r$, has mean value 0 in $I$.

Remark. As before, we allow discontinuities at the discontinuities of $T$. Observe however that the mean value condition implies that the sum of the jumps of $D^{l} \Phi$ $(0 \leq l<r)$ over the discontinuities of $T$ (including the endpoints of $I)$ is zero. 
We will indicate below why the mean value condition is natural.

On the other hand, we will look for solutions in the space $\mathcal{C}^{r-2+\operatorname{Lip}}(I)$ of functions $\Psi$ which are $\mathcal{C}^{r-2}$ on all of $I$, the derivative of order $r-2, D^{r-2} \Psi$ being Lipschitz on $I$. For $r=1$, this is just the space of bounded functions on $I$. Observe that, as soon as $r \geq 2$, we do not allow discontinuities.

3.4.3. For $T$ an i.e.m. of Roth type, denote by $\Gamma_{T}=\Gamma_{T}^{(0)}$ the space of functions $\chi \in \Gamma$ (constant on each $\left.j_{0}\left(I_{\alpha}\right)\right)$ which can be written as

$$
\chi=\psi-\psi \circ T
$$

with bounded $\psi$. This is a linear subspace of $\Gamma$ which is contained in $\Gamma_{*}$ and contains $\Gamma_{s}$. We can rephrase our main theorem by saying that there is a welldefined obstruction map

$$
\mathrm{BV}_{*}^{1}(I) \rightarrow \Gamma / \Gamma_{T}
$$

which associates to $\Phi$ the function in $\Gamma$ we must subtract from $\Phi$ in order to be able to solve the cohomological equation. We recognize (some of) the Forni distribution conditions, by choosing a basis in the finite-dimensional space $\Gamma / \Gamma_{T}$. The number of conditions is just the codimension of $\Gamma_{T}$, as the restriction of the obstruction map to $\Gamma$ is just the quotient map and thus the obstruction map is onto.

3.4.4. Now let $r \geq 1, \Phi \in \mathrm{BV}_{*}^{r}(I)$, and let us try to solve (under finitely many linear conditions on $\Phi)$ the cohomological equation

$$
\Phi=\Psi-\Psi \circ T
$$

with $\Psi \in \mathcal{C}^{r-2+\operatorname{Lip}}(I)$. We assume that the i.e.m. is of Roth type. Consider the $r d$-dimensional space $\Gamma(r)$ of functions $\chi$ on $I$ whose restrictions to each $j_{0}\left(I_{\alpha}\right)$ are polynomials of degree $<r$. For $r=1$, this is our previous space $\Gamma$. Consider also

$$
\Gamma_{*}(r)=\Gamma(r) \cap \mathrm{BV}_{*}^{r}(I),
$$

which has codimension $(r-1)$ in $\Gamma(r)$. We first describe the subspace $\Gamma_{T}(r)$ of $\Gamma_{*}(r)$ of functions $\chi$ which can be written as

$$
\chi=\psi \circ T-\psi
$$

with $\psi \in \mathcal{C}^{r-2+\operatorname{Lip}}(I)$.

Lemma. For $r \geq 1$, the map $\chi \mapsto D \chi$ from $\Gamma(r+1)$ to $\Gamma(r)$ sends $\Gamma_{*}(r+1)$ to $\Gamma_{*}(r)$ and $\Gamma_{T}(r+1)$ to $\Gamma_{T}(r)$. The kernel, i.e. the intersection $\Gamma \cap \Gamma_{T}(r+1)$, is equal to $\mathbb{R} \delta$; we have thus

$$
\operatorname{dim} \Gamma_{T}(r)=\operatorname{dim} \Gamma_{T}+(r-1) .
$$

Proof. It is clear that $\chi \mapsto D \chi$ sends $\Gamma_{*}(r+1)$ to $\Gamma_{*}(r)$ and $\Gamma_{T}(r+1)$ to $\Gamma_{T}(r)$. If $\psi_{0}(x) \equiv x$, then $\psi_{0} \circ T(x)-\psi_{0}(x)=\delta_{\alpha}$ for $x \in j_{0}\left(I_{\alpha}\right)$; hence $\mathbb{R} \delta \subset \Gamma \cap \Gamma_{T}(r)$ for all $r \geq 1$. Conversely, if $\chi \in \Gamma \cap \Gamma_{T}(r)$, write $\chi=\psi \circ T-\psi$ with $\psi \in \operatorname{Lip}(I)$. Taking derivatives, $D \psi$ is $T$-invariant, hence constant as $T$ is ergodic. Therefore $\chi \in \mathbb{R} \delta$.

Theorem. Let $r \geq 1$. For any $\Phi \in B V_{*}^{r}(I)$, one can find $\chi \in \Gamma_{*}(r), \psi \in$ $\mathcal{C}^{r-2+\operatorname{Lip}}(I)$ such that

$$
\Phi=\chi+\Psi \circ T-\Psi
$$


In other terms, the map

$$
\begin{aligned}
\Phi & \mapsto \chi, \\
B V_{*}^{r}(I) & \rightarrow \Gamma_{*}(r) / \Gamma_{T}(r)
\end{aligned}
$$

is the obstruction map associated with the cohomological equation with the prescribed regularities.

Proof. By induction on $r$, the case $r=1$ being our main theorem. Assume $\Phi \in$ $\mathrm{BV}_{*}^{r+1}(I)$. Then $D \Phi \in \mathrm{BV}_{*}^{r}(I)$. By the induction hypothesis, one can write

$$
D \Phi=\chi_{1}+\Psi_{1} \circ T-\Psi_{1}
$$

with $\chi_{1} \in \Gamma_{*}(r)$ and $\Psi_{1} \in \mathcal{C}^{r-2+\operatorname{Lip}}(I)$. Let $\Psi$ be a primitive of $\Psi_{1}, \chi_{0}$ be a primitive of $\chi_{1}$. Then $\psi \in \mathcal{C}^{r-1+\operatorname{Lip}}(I)$. As $D \Phi$ has mean value $0, \chi_{1}$ has also mean value 0 and $\chi_{0} \in \Gamma_{*}(r+1)$. The difference $\chi_{0}^{\prime}=\Phi-\chi_{0}-\Psi \circ T+\Psi$ belongs to $\Gamma$ and we take $\chi=\chi_{0}+\chi_{0}^{\prime}$.

3.4.5. We explain now why the regularities for $\Phi, \Psi$ are "natural".

Let $\zeta=\left(\zeta_{\alpha}\right)_{\alpha \in \mathcal{A}}$ be suspension data, and let $M_{\zeta}$ be the surface constructed from these data as in 3.2 .

Let $\tilde{\Phi}$ be a continuous function on $M_{\zeta}$. With the notation of 3.1, we define, for $\alpha \in \mathcal{A}$ :

$$
\begin{aligned}
& \mathcal{I}_{\alpha}^{0}=\int_{0}^{\Im m \xi_{\alpha}^{0}} \tilde{\Phi}\left(\Re e \xi_{\alpha}^{0}, y\right) d y, \\
& \mathcal{I}_{\alpha}^{1}=\int_{\Im m \xi_{\alpha}^{1}}^{0} \tilde{\Phi}\left(\Re e \xi_{\alpha}^{1}, y\right) d y ;
\end{aligned}
$$

for $\alpha \in \mathcal{A}, x \in j_{0}\left(I_{\alpha}\right)$, we also set

$$
\Phi(x)=\int_{0}^{h_{\alpha}} \tilde{\Phi}(x, y) d y .
$$

Observe that we have

$$
\begin{aligned}
\Phi\left(\left(\Re e \xi_{\alpha}^{0}\right)^{-}\right) & =\mathcal{I}_{\alpha}^{0}+\mathcal{I}_{\alpha}^{1}, \\
\Phi\left(\Re e \xi_{\alpha}^{0}-\lambda_{\alpha}\right) & =\mathcal{I}_{\beta_{0}}^{0}+\mathcal{I}_{\beta_{1}}^{1},
\end{aligned}
$$

where $\pi_{0}\left(\beta_{0}\right)+1=\pi_{0}(\alpha), \pi_{1}\left(\beta_{1}\right)+1=\pi_{1}(\alpha)$, except if $\pi_{0}(\alpha)=1$ (respectively $\left.\pi_{1}(\alpha)=1\right)$ when $\mathcal{I}_{\beta_{0}}^{0}\left(\right.$ resp. $\left.\mathcal{I}_{\beta_{1}}^{1}\right)$ is declared to be 0 .

From these formulas and $\mathcal{I}_{\alpha_{0}}^{0}+\mathcal{I}_{\alpha_{1}}^{1}=0$ (with $\pi_{\varepsilon}\left(\alpha_{\varepsilon}\right)=d$ as usual), we obtain

$$
\sum_{\alpha \in \mathcal{A}} \Phi\left(\left(\Re e \xi_{\alpha}^{0}\right)^{-}\right)=\sum_{\alpha \in \mathcal{A}} \Phi\left(\Re e \xi_{\alpha}^{0}-\lambda_{\alpha}\right),
$$

which means that the derivative of $\Phi$ (when it exists) has mean value 0 . This explains the conditions defining $\mathrm{BV}_{*}^{r}(I)$. On the other hand, if $\tilde{\Psi}$ is a function on $M_{\zeta}$ satisfying

$$
\frac{\partial}{\partial y} \tilde{\Psi}=\tilde{\Phi}
$$

and we define

$$
\Psi(x)=\tilde{\Psi}(x, 0)
$$

then we will have

$$
\Psi \circ T-\Psi=\Phi .
$$




\section{Proof of full measure for Roth type}

We will first recall the construction of the finite measure, absolutely continuous w.r.t. Lebesgue measure, which is invariant under the Zorich algorithm (normalized).

4.1. The basic operation of the algorithm for suspensions. Let $\left(\mathcal{A}, \pi_{0}, \pi_{1}\right)$ and $\left(\zeta_{\alpha}=\lambda_{\alpha}+i \tau_{\alpha}\right)_{\alpha \in \mathcal{A}}$ be as above. Construct $R_{\zeta}, M_{\zeta}$ as in 3.2 and 3.3. With $\pi_{\varepsilon}\left(\alpha_{\varepsilon}\right)=d$ as above, assume that

$$
\lambda_{\alpha_{0}} \neq \lambda_{\alpha_{1}}
$$

Then the formula $\lambda_{\alpha_{\varepsilon}}=\operatorname{Max}\left(\lambda_{\alpha_{0}}, \lambda_{\alpha_{1}}\right)$ defines uniquely $\varepsilon \in\{0,1\}$ and determines uniquely the basic step of the continued fraction algorithm; this step produces new combinatorial data $\left(\mathcal{A}, \hat{\pi}_{0}, \hat{\pi}_{1}\right)$ and length data $\left(\hat{\lambda}_{\alpha}\right)_{\alpha \in \mathcal{A}}$ given by

$$
\begin{gathered}
\hat{\lambda}_{\alpha}=\lambda_{\alpha}, \quad \alpha \neq \alpha_{\varepsilon}, \\
\hat{\lambda}_{\alpha_{\varepsilon}}=\lambda_{\alpha_{\varepsilon}}-\lambda_{\alpha_{1-\varepsilon}} .
\end{gathered}
$$

For suspension data, we just define in the same way

$$
\begin{gathered}
\hat{\zeta}_{\alpha}=\zeta_{\alpha}, \quad \alpha \neq \alpha_{\varepsilon}, \\
\hat{\zeta}_{\alpha_{\varepsilon}}=\zeta_{\alpha_{\varepsilon}}-\zeta_{\alpha_{1-\varepsilon}} .
\end{gathered}
$$

This has a nice representation in terms of the corresponding regions $R_{\zeta}, R_{\hat{\zeta}}$. One cuts from $R_{\zeta}$ the part where $x \geq \hat{\lambda}^{*}=\lambda^{*}-\lambda_{\alpha_{\varepsilon}}$ : it is made of $R_{\alpha_{1-\varepsilon}}^{1-\varepsilon}$ and a right part of $R_{\alpha_{\varepsilon}}^{\varepsilon}$. We glue back $R_{\alpha_{1-\varepsilon}}^{1-\varepsilon}$ to the free horizontal side of $R_{\alpha_{\varepsilon}}^{1-\varepsilon}$, and the right part of $R_{\alpha_{\varepsilon}}^{\varepsilon}$ to $R_{\alpha_{1-\varepsilon}}^{\varepsilon}$; see Figure 4 .

It is easy to check that the new suspension data satisfy the inequalities required in 3.1 ; if for instance $\varepsilon=0$, one has

$$
\hat{\xi}_{\alpha}^{0}=\xi_{\alpha}^{0}, \alpha \neq \alpha_{0}
$$

with $\hat{\pi}_{0}=\pi_{0}$ on one hand and

$$
\begin{aligned}
& \hat{\xi}_{\alpha}^{1}=\xi_{\alpha}^{1}, \alpha \neq \alpha_{0}, \alpha_{1}, \\
& \hat{\xi}_{\alpha_{1}}^{1}=\xi_{\alpha_{0}}^{1}, \\
& \hat{\xi}_{\alpha_{0}}^{1}=\xi_{\alpha_{0}}^{1}-\zeta_{\alpha_{1}} .
\end{aligned}
$$

The last formula gives

$$
\begin{aligned}
-\hat{\xi}_{\alpha_{0}}^{1} & =\zeta_{\alpha_{1}}-\xi_{\alpha_{0}}^{1} \\
& =\zeta_{\alpha_{1}}-\xi_{\alpha_{0}}^{0}-\theta_{\alpha_{0}} \\
& =\zeta_{\alpha_{1}}-\xi_{\alpha_{1}}^{1}-\theta_{\alpha_{0}} \\
& =-\xi_{\tilde{\alpha}_{1}}^{1}-\theta_{\alpha_{0}}
\end{aligned}
$$

with $\pi_{1}\left(\tilde{\alpha}_{1}\right)=d-1$. We therefore have

$$
-\Im m \hat{\xi}_{\alpha_{0}}^{1}=-\Im m \xi_{\tilde{\alpha}_{1}}^{1}+h_{\alpha_{0}}>0 .
$$

We also see that ( still with $\varepsilon=0$ ), if $\hat{\alpha}_{1} \in \mathcal{A}$ is such that $\hat{\pi}_{1}\left(\hat{\alpha}_{1}\right)=d$ (we have $\hat{\alpha}_{1}=\tilde{\alpha}_{1}$ if $\tilde{\alpha}_{1} \neq \alpha_{0}, \hat{\alpha}_{1}=\alpha_{1}$ if $\left.\tilde{\alpha}_{1}=\alpha_{0}\right)$, one has

$$
\Im m \hat{\xi}_{\hat{\alpha}_{1}}^{1}=\Im m \xi_{\tilde{\alpha}_{1}}^{1}<0 \text {. }
$$



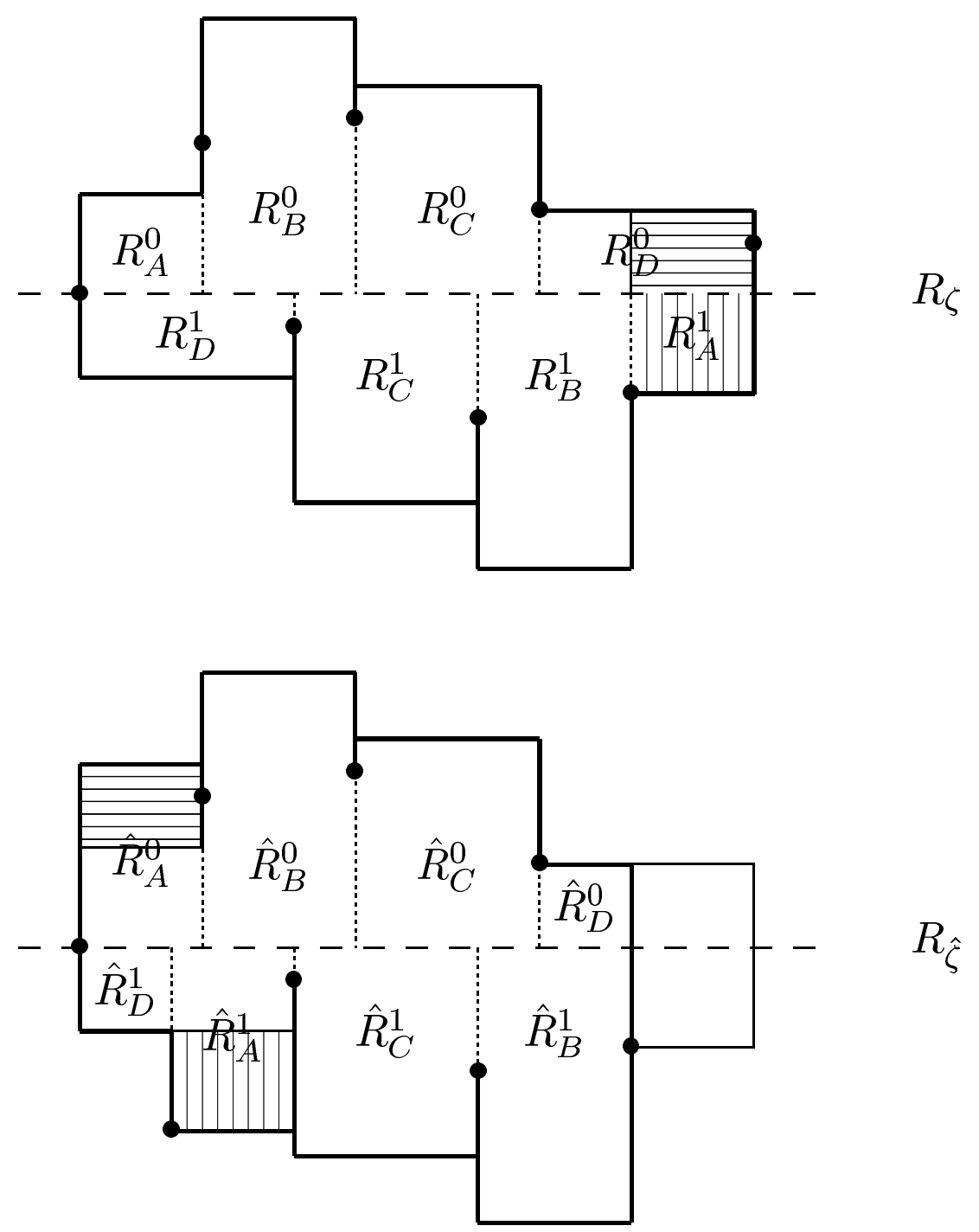

Figure 4. The Rauzy-Veech operation for suspensions

Conversely, given $\left(\mathcal{A}, \pi_{0}, \pi_{1}\right)$ and $\left(\zeta_{\alpha}=\lambda_{\alpha}+i \tau_{\alpha}\right)_{\alpha \in \mathcal{A}}$ as above, assume that

$$
\Im m \xi_{\alpha_{0}}^{0}=\Im m \xi_{\alpha_{1}}^{1} \neq 0 \text {, }
$$

and define $\varepsilon=0$ if $\Im m \xi_{\alpha_{1}}^{1}<0, \varepsilon=1$ if $\Im m \xi_{\alpha_{0}}^{0}>0$. Set

$$
\begin{gathered}
\hat{\zeta}_{\alpha}=\zeta_{\alpha}, \quad \alpha \neq \alpha_{\varepsilon}, \\
\hat{\zeta}_{\alpha_{\varepsilon}}=\zeta_{\alpha_{\varepsilon}}+\zeta_{\alpha_{1-\varepsilon}},
\end{gathered}
$$

and define appropriately new combinatorial data; this operation is the inverse of the one above. Thus the dynamics of the continued fraction algorithm at the level of suspension is invertible (on a full measure set) and can be viewed as the natural extension of the dynamics at the level of i.e.m. 
It is clear that the Riemann surfaces $M_{\zeta}, M_{\hat{\zeta}}$ are canonically isomorphic, and the isomorphism respects the holomorphic 1-form and the vertical vector field.

We can also extend the definition of the Zorich algorithm at the level of suspension data. These accelerated dynamics can actually be thought of as a first return map of the previous dynamics. Indeed, in the polyhedral cone of admissible length and suspension data, consider the polyhedral subcones defined by

$$
\begin{aligned}
& \mathcal{Z}_{0}=\left\{\lambda_{\alpha_{0}}>\lambda_{\alpha_{1}}, \Im m \xi_{\alpha_{0}}^{0}>0\right\}, \\
& \mathcal{Z}_{1}=\left\{\lambda_{\alpha_{1}}>\lambda_{\alpha_{0}}, \Im m \xi_{\alpha_{1}}^{1}<0\right\} .
\end{aligned}
$$

The accelerated dynamics are the first return map to $\mathcal{Z}=\mathcal{Z}_{0} \sqcup \mathcal{Z}_{1}$; this is clear from the description of the basic step above.

4.2. The Teichmüller flow. Fix combinatorial data $\left(\mathcal{A}, \pi_{0}, \pi_{1}\right)$. Given length data $\left(\lambda_{\alpha}\right)_{\alpha \in \mathcal{A}}$ and suspension data $\left(\tau_{\alpha}\right)_{\alpha \in \mathcal{A}}$, one defines for $t \in \mathbb{R}$,

$$
U^{t}(\lambda, \tau)=\left(e^{t / 2} \lambda, e^{-t / 2} \tau\right) .
$$

This flow is called the Teichmüller flow. Observe that the conditions on the length data $\lambda_{\alpha}>0$ and on the suspension data (see 3.1) are preserved under the flow.

It is also obvious that the flow commutes with the basic operation of the continued fraction algorithm. In particular, the inequality $\lambda_{\alpha_{\varepsilon}}>\lambda_{\alpha_{1-\varepsilon}}$ is preserved.

The surface $M_{\zeta}$ is canonically equipped with an area form (coming from $\mathbb{C}$ ) for which its area is

$$
A:=\operatorname{area}\left(M_{\zeta}\right)=\sum_{\alpha \in \mathcal{A}} \lambda_{\alpha} h_{\alpha} .
$$

The area is preserved by the Teichmüller flow, and also by the basic operation of the continued fraction algorithm.

The Lebesgue measure $d \lambda d \tau$ on the domain $\mathbb{R}^{\mathcal{A}} \times \mathbb{R}^{\mathcal{A}}$ defined by the restrictions on length and suspension data is preserved by the Teichmüller flow, and by the basic operation of the continued fraction algorithm.

One now combines the continued fraction algorithm (in Zorich form) with the Teichmüller flow in order to get a version which is normalized w.r.t. scales.

One could decide to normalize by keeping the total length $\lambda^{*}=\sum_{\alpha \in \mathcal{A}} \lambda_{\alpha}$ constant; actually, we prefer in the sequel a slightly different normalization, which leads to simpler formulas.

As in 1.2.1, for $\lambda_{\alpha_{\varepsilon}}>\lambda_{\alpha_{1-\varepsilon}}$, we set

$$
\begin{array}{r}
\hat{\lambda}_{\alpha}=\lambda_{\alpha}, \quad \alpha \neq \alpha_{\varepsilon}, \\
\hat{\lambda}_{\alpha_{\varepsilon}}=\lambda_{\alpha_{\varepsilon}}-\lambda_{\alpha_{1-\varepsilon}} .
\end{array}
$$

Define

$$
\hat{\lambda}^{*}=\sum_{\alpha \in \mathcal{A}} \hat{\lambda}_{\alpha}=\lambda^{*}-\lambda_{\alpha_{1-\varepsilon}},
$$

as in 1.2.1. Let $\left(\pi_{0}, \pi_{1}, \lambda, \tau\right)$ belong to the domain $\mathcal{Z}$ of the Zorich algorithm, and let $\left(\bar{\pi}_{0}, \bar{\pi}_{1}, \bar{\lambda}, \bar{\tau}\right)$ be the image. Define

$$
\begin{aligned}
t & =t(\lambda)=2\left(\log \hat{\lambda}^{*}-\log \hat{\bar{\lambda}}^{*}\right), \\
\bar{G}\left(\pi_{0}, \pi_{1}, \lambda, \tau\right) & =\left(\bar{\pi}_{0}, \bar{\pi}_{1}, U^{t(\lambda)}(\bar{\lambda}, \bar{\tau})\right) .
\end{aligned}
$$

The map $\bar{G}$ is called the normalized step for the natural extension of the accelerated algorithm. 
4.3. The absolutely continuous invariant measure. We already observed that the restriction of Lebesgue measure $d \lambda d \tau$ to the simplicial cone of admissible length and suspension data is invariant under both the basic step of the algorithm and the Teichmüller flow.

When we further restrict Lebesgue measure to $\mathcal{Z}$, we obtain a measure $m_{0}$ which is still invariant under Teichmüller flow and is now invariant under the accelerated algorithm.

Observe that the function $t$ used in the definition of $\bar{G}$ is constant along the orbits of the Teichmüller flow. It follows that the measure $m_{0}$ is also invariant under $\bar{G}$.

The area function $A=\sum_{\alpha \in \mathcal{A}} \lambda_{\alpha} h_{\alpha}$ (where $h=-\Omega \tau$ ) is also invariant under $\bar{G}$; we introduce

$$
\mathcal{Z}^{(1)}=\mathcal{Z} \cap\{A \leq 1\},
$$

and denote by $m_{1}$ the restriction of $m_{0}$ to $\mathcal{Z}^{(1)}$; it is invariant under the restriction of $\bar{G}$ to $\mathcal{Z}^{(1)}$.

We now project back to the level of i.e.m., i.e., of length data alone: we obtain a map

$$
G\left(\pi_{0}, \pi_{1}, \lambda\right)=\left(\bar{\pi}_{0}, \bar{\pi}_{1}, e^{t(\lambda) / 2} \bar{\lambda}\right)
$$

and a measure $m_{2}$, which is the image of $m_{1}$ under the projection, which is invariant under $G$. As $\hat{\lambda}^{*}$ is still invariant under $G$, we can restrict, by homogeneity, the measure $m_{2}$ to $\left\{\hat{\lambda}^{*}=1\right\}$ to obtain a measure $m$ invariant under the restriction of $G$. This is the measure that we are interested in and that we will now describe.

Let $\left(\pi_{0}, \pi_{1}, \lambda\right)$ be fixed; assume for instance that $\lambda_{\alpha_{0}}>\lambda_{\alpha_{1}}$. Consider in $\tau$-space the polyhedral cone

$$
\mathcal{U}_{0}=\left\{\Im m \xi_{\alpha}^{0}>0, \forall \alpha \in \mathcal{A}, \Im m \xi_{\alpha}^{1}<0, \forall \alpha \neq \alpha_{1}\right\} .
$$

The density $\chi$ of $m_{2}$ at $\left(\pi_{0}, \pi_{1}, \lambda\right)$ is equal to the volume of $\mathcal{U}_{0} \cap\{A \leq 1\}$. Write $\mathcal{U}_{0}$, up to a codimension 1 subset, as a finite union of disjoint simplicial cones $\mathcal{U}$. For each $\mathcal{U}$, choose a unimodular basis $\tau^{(1)}, \ldots, \tau^{(d)}$ of $\mathbb{R}^{\mathcal{A}}$ generating $\mathcal{U}$ and write $h^{(j)}=-\Omega \tau^{(j)}$. One has

$$
\chi_{\pi_{0}, \pi_{1}}(\lambda)=(d !)^{-1} \sum_{\mathcal{U}} \prod_{1}^{d}\left(\sum_{\alpha \in \mathcal{A}} \lambda_{\alpha} h_{\alpha}^{(j)}\right)^{-1} .
$$

If we set

$$
\begin{aligned}
\hat{\lambda}_{\alpha_{0}} & =\lambda_{\alpha_{0}}-\lambda_{\alpha_{1}}, \\
\hat{\lambda}_{\alpha} & =\lambda_{\alpha}, \alpha \neq \alpha_{0}, \\
\hat{h}_{\alpha_{1}} & =h_{\alpha_{0}}+h_{\alpha_{1}}, \\
\hat{h}_{\alpha} & =h_{\alpha}, \alpha \neq \alpha_{1},
\end{aligned}
$$

we have

$$
\sum_{\alpha \in \mathcal{A}} \lambda_{\alpha} h_{\alpha}^{(j)}=\sum_{\alpha \in \mathcal{A}} \hat{\lambda}_{\alpha} \hat{h}_{\alpha}^{(j)}
$$

Define

$$
W_{j}=\left\{\alpha \in \mathcal{A}, \hat{h}_{\alpha}^{(j)} \neq 0\right\} .
$$

The key property is now the following ([V2], [Z1]; see also $[\mathrm{Y}]$ ). 
Lemma. For any $X \subset \mathcal{A}$ with $\emptyset \neq X \neq \mathcal{A}$, we have

$$
\operatorname{card}\left\{j, W_{j} \cap X=\emptyset\right\}+\operatorname{card} X<d .
$$

When $\lambda_{\alpha_{1}}>\lambda_{\alpha_{0}}$, the only difference is that we have to start with

$$
\mathcal{U}_{1}=\left\{\Im m \xi_{\alpha}^{0}>0, \forall \alpha \neq \alpha_{0}, \Im m \xi_{\alpha}^{1}<0, \forall \alpha \in \mathcal{A}\right\} .
$$

In the formula $(*)$ above for the density, set

$$
\chi_{\mathcal{U}}(\lambda)=\prod_{1}^{d}\left(\sum_{\alpha \in \mathcal{A}} \lambda_{\alpha} h_{\alpha}^{(j)}\right)^{-1} .
$$

Up to a constant factor, the density of $m$ on the simplex

$$
\Delta=\left\{\lambda \in \mathbb{R}^{\mathcal{A}}, \hat{\lambda}_{\alpha}>0, \hat{\lambda}^{*}=1\right\}
$$

is given by $\sum_{\mathcal{U}} \chi_{\mathcal{U}}$. One has

$$
c^{-1} \leq \chi_{\mathcal{U}}(\lambda) \prod_{j=1}^{d}\left(\sum_{W_{j}} \hat{\lambda}_{\alpha}\right) \leq c .
$$

To control the size of $\chi \mathcal{U}$, we decompose $\Delta$ as follows. Set

$$
\mathcal{N}=\left\{\vec{n}=\left(n_{\alpha}\right)_{\alpha \in \mathcal{A}} \in \mathbb{N}^{\mathcal{A}}, \min _{\alpha} n_{\alpha}=0\right\} .
$$

For $\vec{n} \in \mathcal{N}, \Delta(\vec{n})$ is the set of $\lambda \in \Delta$ such that

$$
\begin{gathered}
\hat{\lambda}_{\alpha} \geq \frac{1}{2 d} \text { if } n_{\alpha}=0, \\
\frac{1}{2 d} 2^{1-n_{\alpha}}>\hat{\lambda}_{\alpha} \geq \frac{1}{2 d} 2^{-n_{\alpha}} \text { if } n_{\alpha}>0 .
\end{gathered}
$$

We obtain thus a partition

$$
\Delta=\bigsqcup_{\mathcal{N}} \Delta(\vec{n})
$$

with the estimate

$$
c^{-1} \leq 2^{\Sigma n_{\alpha}} \operatorname{vol} \Delta(\vec{n}) \leq c .
$$

For $\lambda \in \Delta(\vec{n})$, estimate (1) above gives

$$
c^{-1} \leq \chi_{\mathcal{U}}(\lambda) 2^{-\Sigma_{j} \min _{W_{j}} n_{\alpha}} \leq c .
$$

With fixed $\vec{n}$, let $0=n^{0}<n^{1}<\ldots$ be the values taken by the $n_{\alpha}$ and $V^{i} \subset \mathcal{A}$ the set of indices with $n_{\alpha} \geq n^{i}$. On one side, one has

$$
\begin{aligned}
\sum_{\alpha \in \mathcal{A}} n_{\alpha} & =\sum_{i \geq 0} n^{i}\left(\operatorname{card}\left(V^{i} \backslash V^{i+1}\right)\right) \\
& =\sum_{i>0}\left(n^{i}-n^{i-1}\right) \operatorname{card} V^{i} .
\end{aligned}
$$

On the other side, let $\tilde{V}^{i}$ be the set of $j$ such that $W_{j} \subset V^{i}$; one has $\min _{W_{j}} n_{\alpha}=n^{i}$ if and only if $j \in \tilde{V}^{i} \backslash \tilde{V}^{i+1}$; hence

$$
\begin{aligned}
\sum_{j=1}^{d} \min _{W_{j}} n_{\alpha} & =\sum_{i \geq 0} n^{i}\left(\operatorname{card}\left(\tilde{V}^{i} \backslash \tilde{V}^{i+1}\right)\right) \\
& =\sum_{i>0}\left(n^{i}-n^{i-1}\right) \operatorname{card} \tilde{V}^{i} .
\end{aligned}
$$


By the lemma above, one has

$$
\operatorname{card} \tilde{V}^{i}<\operatorname{card} V^{i}
$$

as long as $0<\operatorname{card} V^{i}<d$. This shows that

$$
\sum_{\alpha \in \mathcal{A}} n_{\alpha}-\sum_{j=1}^{d} \min _{W_{j}} n_{\alpha} \geq|\vec{n}|_{\infty}:=\operatorname{Max}_{\alpha \in \mathcal{A}} n_{\alpha} .
$$

The last estimate, introduced into (2), (3) gives

$$
(\operatorname{vol} \Delta(\vec{n})) \operatorname{Max}_{\Delta(\vec{n})} \chi \mathcal{U} \leq c 2^{-|\vec{n}|_{\infty}} .
$$

The integrability of $\chi_{\mathcal{U}}$ over $\Delta$ now follows from the fact that the number of $\vec{n} \in \mathcal{N}$ with $|\vec{n}|_{\infty}=N$ is of order $N^{d-2}$.

If we compare (2) and (4), we obtain

$$
\operatorname{Max}_{\Delta(\vec{n})} \chi_{\pi_{0}, \pi_{1}} \leq c 2^{|\vec{n}|_{1}-|\vec{n}|_{\infty}}
$$

with $|\vec{n}|_{1}=\sum_{\alpha \in \mathcal{A}} n_{\alpha}$. When $d=2, \chi_{\pi_{0}, \pi_{1}}$ is bounded. Assume now $d>2$. From (2) and (5), one obtains

$$
m\left(\left\{\chi_{\pi_{0}, \pi_{1}}>2^{N}\right\}\right) \leq \sum_{|\vec{n}|_{1}-|\vec{n}|_{\infty} \geq N-c} c 2^{-|\vec{n}|_{\infty}} ;
$$

to have $|\vec{n}|_{1} \geq|\vec{n}|_{\infty}+N-c$, one must have $|\vec{n}|_{\infty} \geq \frac{N-c}{d-2}$; an easy computation leads to

$$
\begin{gathered}
m\left(\left\{\chi_{\pi_{0}, \pi_{1}}>2^{N}\right\}\right) \leq c 2^{-\frac{N}{d-2}}, \\
\operatorname{Leb}\left(\left\{\chi_{\pi_{0}, \pi_{1}}>2^{N}\right\}\right) \leq c 2^{-N \frac{d-1}{d-2}} .
\end{gathered}
$$

It follows, as $\chi_{\pi_{0}, \pi_{1}}$ is bounded from below, that we have, for every Borel set $X$,

$$
c^{-1} \operatorname{Leb}(X) \leq m(X) \leq c(\operatorname{Leb}(X))^{\frac{1}{d-1}} .
$$

4.4. Integrability of $\log \left\|Z_{(1)}\right\|$. Recall the function $Z_{(1)}$, with values in $\operatorname{SL}\left(\mathbb{Z}^{\mathcal{A}}\right)$, defined in 1.2.4: the sequence $\left(\pi_{0}^{(k)}, \pi_{1}^{(k)}, \lambda^{(k)}\right)$ given by the Zorich algorithm satisfies

$$
\lambda^{(k)}=Z_{(1)}\left(\pi_{0}^{(k)}, \pi_{1}^{(k)}, \lambda^{(k)}\right) \lambda^{(k+1)} .
$$

Following Zorich ([Z1]) we estimate $\left\|Z_{(1)}\right\|$ w.r.t. the absolutely continuous invariant measure $m$. This will be used in two ways:

- applying Oseledets' multiplicative ergodic theorem in order to prove that conditions (b) and (c) in 1.3 have full measure;

- as a first step in an induction to prove that condition (a) in 1.3.1 has full measure.

We use as norm the supremum of the coefficients. For $k \geq 0, \lambda_{\alpha_{\varepsilon}}>\lambda_{\alpha_{1-\varepsilon}}$, we have

$$
\left\|Z_{(1)}\right\|>k \Longleftrightarrow \hat{\lambda}_{\alpha_{\varepsilon}}>k \sum_{\pi_{1-\varepsilon} \alpha>\pi_{1-\varepsilon} \alpha_{\varepsilon}} \hat{\lambda}_{\alpha}
$$

it follows that

$$
\left\|Z_{(1)}\right\|>(2 d) 2^{N-1} \Rightarrow \lambda \in \bigcup_{|\vec{n}|_{\infty} \geq N} \Delta(\vec{n}),
$$

which in turn implies that

$$
m\left(\left\{\left\|Z_{(1)}\right\|>2^{N}\right\}\right) \leq c N^{d-2} 2^{-N} .
$$


This is the required estimate; it shows that $\left\|Z_{(1)}\right\|^{\rho}$ is $m$-integrable for all $\rho<1$ and a fortiori that $\log \left\|Z_{(1)}\right\|$ is $m$-integrable.

4.5. Conditions (b) and (c) have full measure. As $\log \left\|Z_{(1)}\right\|$ is $m$-integrable, we can apply Oseledets' theorem and obtain the existence almost everywhere of Lyapunov exponents for the corresponding cocycle.

The space $\Gamma_{s}$ is then associated to the negative Lyapunov exponents. The two estimates in condition (c) are immediate consequences of the properties of Oseledets' decomposition.

For property (b), we recall the result of Veech ( $\mathrm{V} 3])$ : the largest Lyapunov exponent is almost everywhere simple. The existence of a spectral gap follows.

At the end of the section, we will prove that property (a) in 1.3.1 has full measure.

4.6. The main step. Let $\left(\mathcal{A}, \pi_{0}, \pi_{1}\right)$ be combinatorial data, $\mathcal{D}$ the associated Rauzy diagram. For an i.e.m. $T$ satisfying Keane's condition with these data, the Rauzy-Veech algorithm defines an infinite path $\left(\gamma^{(n)}(T)\right)_{n>0}$ in $\mathcal{D}$, starting at $\left(\pi_{0}, \pi_{1}\right)$.

Conversely, if $\gamma=\left(\gamma^{(n)}\right)_{0<n \leq N}$ is a finite path in $\mathcal{D}$ starting at $\left(\pi_{0}, \pi_{1}\right)$, we denote by $\Delta(\gamma)$ the simplex of normalized $T$ in $\Delta\left(\pi_{0}, \pi_{1}\right)$ such that $\gamma^{(n)}(T)=\gamma^{(n)}$ for $0<n \leq N$. We use here the old normalization $\left\{\lambda^{*}=1\right\}$.

To such a path $\gamma$ is associated a matrix $Q(\gamma) \in \mathrm{SL}\left(\mathbb{Z}^{\mathcal{A}}\right)$ :

$$
Q(\gamma)=V\left(\gamma^{(1)}\right) \cdots V\left(\gamma^{(n)}\right)
$$

and we write as before

$$
Q_{\beta}(\gamma)=\sum_{\alpha \in \mathcal{A}} Q_{\alpha \beta}(\gamma)
$$

We have

$$
1=\sum_{\alpha \in \mathcal{A}} \lambda_{\alpha}^{(0)}=\sum_{\beta \in \mathcal{A}} Q_{\beta}(\gamma) \lambda_{\beta}
$$

(where $\left.\lambda^{(0)}=Q(\gamma) \lambda^{(N)}\right)$, and it follows that

$$
\operatorname{vol}_{d-1}(\Delta(\gamma))=\left[\prod_{\beta \in \mathcal{A}} Q_{\beta}(\gamma)\right]^{-1} \operatorname{vol}_{d-1}\left(\Delta\left(\pi_{0}, \pi_{1}\right)\right) .
$$

Denote by $\left(\pi_{0}^{(N)}, \pi_{1}^{N)}\right)$ the endpoint of $\gamma$, by $\alpha_{0}^{(N)}, \alpha_{1}^{(N)}$ the indices such that $\pi_{\varepsilon}^{(N)}\left(\alpha_{\varepsilon}^{(N)}\right)=d$. They are the names of the two arrows going out of $\left(\pi_{0}^{(N)}, \pi_{1}^{N)}\right)$. The conditional probability, for an i.e.m. $T$ in $\Delta(\gamma)$, that the name of $\gamma^{(N+1)}(T)$ is $\alpha_{\varepsilon}^{(N)}$ is equal to $Q_{\alpha_{1-\varepsilon}^{(N)}}\left(Q_{\alpha_{0}^{(N)}}+Q_{\alpha_{1}^{(N)}}\right)^{-1}$.

Let $1 \leq D<d$. A segment $\left(\gamma^{(n)}(T)\right)_{k \leq n<l}$ is called a $D$-segment if the arrows of the segment take no more than $D$ distinct names. It is called maximal if $\left(\gamma^{(n)}(T)\right)_{k \leq n \leq l}$ is not a $D$-segment.

The following proposition is the main step in proving that condition (a) has full measure.

Proposition. There exist an integer $l=l(d)$ and a constant $\eta=\eta(d)>0$ with the following properties. Let $\gamma=\left(\gamma^{(n)}\right)_{0<n \leq N}$ be a finite path in $\mathcal{D}$ such that the set $\mathcal{A}^{\prime}$ of names of arrows of $\gamma$ is distinct from $\mathcal{A}$. Assume that $D=\operatorname{card} \mathcal{A}^{\prime}>1$. There is a subset $\Delta^{\prime}(\gamma)$ of $\Delta(\gamma)$ with

$$
\operatorname{vol}_{d-1}\left(\Delta^{\prime}(\gamma)\right) \geq \eta \operatorname{vol}_{d-1}(\Delta(\gamma))
$$


such that, for every $T \in \Delta^{\prime}(\gamma)$, there exists $M>N$ with

- the name of $\gamma^{(M)}(T)$ does not belong to $\mathcal{A}^{\prime}$;

- no more than $l(D-1)$-segments are needed to cover $\left(\gamma^{(n)}\right)_{N \leq n<M}$.

We will first explain how the full measure estimate for condition (a) follows from the proposition, and then prove the proposition.

4.7. Condition (a) has full measure. For $T \in \Delta\left(\pi_{0}, \pi_{1}\right)$, satisfying Keane's condition, and $1 \leq D<d$, denote by $Z_{(D)}(T)$ the matrix in $\mathrm{SL}\left(\mathbb{Z}^{\mathcal{A}}\right)$ associated with the initial maximal $D$-segment in $\left(\gamma^{(n)}(T)\right)_{n>0}$. Denote by $M_{(D)}(T)$ (resp. $\left.M_{(D)}^{1}(T)\right)$ the minimal number of $(D-1)$-segments (resp. 1-segments) needed to cover this initial maximal $D$-segment.

Corollary. Let $N>0$. Except on a set of measure $\leq c 2^{-c N^{1 / D}}$, one has

$$
\begin{aligned}
\left\|Z_{(D)}(T)\right\| & \leq 2^{N}, \\
M_{(D)}(T) & \leq N^{1 / D}, \\
M_{(D)}^{1}(T) & \leq N^{\frac{D-1}{D}} .
\end{aligned}
$$

Remark. The measure referred to can be either Lebesgue or the invariant measure $m$ : in view of the last formula of 4.3 , it changes only the values of the constants.

Proof. The estimate for $Z_{(1)}$ has been shown in 4.5. Let us show the estimate for $M_{(D)}$.

Let $\gamma=\left(\gamma^{(n)}\right)_{0<n \leq N}$ be any finite path such that $\left(\gamma^{(n)}\right)_{0<n<N}$ is a $(D-1)$ segment but $\gamma$ is not. First apply the proposition once in each $\Delta(\gamma)$. One obtains that

$$
\operatorname{Leb}\left(\left\{M_{(D)}>l+1\right\}\right)<1-\eta .
$$

We next subdivide the set $\left\{M_{(D)}>l+1\right\}$ into simplices $\Delta\left(\gamma_{1}\right)$, where $\gamma_{1}=$ $\left(\gamma_{1}^{(n)}\right)_{0<n \leq N_{1}}$ is a $D$-segment and $\left(\gamma_{1}^{(n)}\right)_{0<n<N_{1}}$ is the concatenation of $(l+1)$ maximal $(D-1)$-segments. Applying once again the proposition in each $\Delta\left(\gamma_{1}\right)$ gives

$$
\operatorname{Leb}\left(\left\{M_{(D)}>2 l+1\right\}\right)<(1-\eta)^{2} .
$$

Iterating this process leads to the required estimate for $M_{(D)}$.

We next show by induction on $D$ that

$$
m\left(M_{(D)}^{1}>N^{\frac{D-1}{D}}\right) \leq c 2^{-c N^{1 / D}} .
$$

For $D=2$, one has $M_{(D)}=M_{(D)}^{1}$; the comparison between $m$ and the Lebesgue measure gives the estimate. Assume $D>2$ and write

$$
Z_{(D)}(T)=Z_{(D-1)}\left(T_{0}\right) Z_{(D-1)}\left(T_{1}\right) \cdots Z_{(D-1)}^{*}\left(T_{M-1}\right)
$$

with $T_{0}=T, M=M_{(D)}(T)$, and $T_{i}$ is obtained from $T_{0}$ by $n_{i}$ iterations of the Zorich algorithm (we have $0=n_{0}<n_{1}<n_{2}<\ldots$ ); $Z_{(D-1)}^{*}\left(T_{M-1}\right)$ denotes some initial part in the product giving $Z_{(D-1)}\left(T_{M-1}\right)$.

Neglecting a set of measure $\leq c 2^{-c N^{1 / D}}$, we can assume that $M \leq N^{1 / D}$.

By the induction hypothesis, applied with $N^{\prime}=N^{\frac{D-1}{D}}$, we have

$$
m\left(M_{(D-1)}^{1}\left(T_{0}\right)>N^{\frac{D-2}{D}}\right) \leq c 2^{-c N^{1 / D}} .
$$


As the measure $m$ is invariant under the Zorich algorithm, the same estimate holds when we put instead of $T_{0}$ any given iterate $T^{(k)}$ of $T_{0}$ under the algorithm. Thus we have

$$
\begin{aligned}
m\left(\operatorname{Max}_{0 \leq k<N} M_{(D-1)}^{1}\left(T^{(k)}\right)>N^{\frac{D-2}{D}}\right) & \leq c N 2^{-c N^{1 / D}} \\
& \leq c^{\prime} 2^{-c^{\prime} N^{1 / D}} .
\end{aligned}
$$

On the other side, when

$$
\operatorname{Max}_{0 \leq k<N} M_{(D-1)}^{1}\left(T^{(k)}\right) \leq N^{\frac{D-2}{D}},
$$

we have

$$
n_{i} \leq i N^{\frac{D-2}{D}}
$$

for

$$
0 \leq i \leq M-1<N^{1 / D}
$$

and

$$
M_{(D)}^{1}(T) \leq N^{\frac{D-1}{D}} .
$$

This proves the estimate for $M_{(D)}^{1}$.

The estimate on $Z_{(D)}$ is again proven by induction on $D$, the case $D=1$ having been done in 4.4. Neglecting a set of measure $c 2^{-c N^{1 / D}}$, we may assume $M_{(D)}(T) \leq$ $N^{1 / D}$ and $M_{(D)}^{1}(T) \leq N^{\frac{D-1}{D}}$. Write $Z_{(D)}(T)$ as above.

If $\left\|Z_{(D)}(T)\right\|>2^{N}$, one can find $i \in\{0,1, \ldots, M-1\}$ such that

$$
\left\|Z_{(D-1)}\left(T_{i}\right)\right\|>2^{N / M} \geq 2^{N^{\frac{D-1}{D}}} .
$$

By the induction hypothesis, we have

$$
m\left(\left\{\left\|Z_{(D-1)}\left(T_{0}\right)\right\|>2^{N^{\frac{D-1}{D}}}\right) \leq c 2^{-c N^{1 / D}}\right.
$$

and the same estimate holds if we replace $T_{0}$ by any given $T^{(k)}$. It is sufficient to consider $k \leq N^{\frac{D-1}{D}}$. Again, one has

$$
c N^{\frac{D-1}{D}} 2^{-c N^{1 / D}} \leq c^{\prime} 2^{-c^{\prime} N^{1 / D}},
$$

and this concludes the proof of the corollary.

The proof that condition (a) has full measure follows now from a usual BorelCantelli argument. Take $D=d-1$ and write $N=(\kappa \log k)^{d-1}$ with fixed large $\kappa>0$ and an integer $k \geq 0$. One has

$$
m\left(\left\{\left\|Z_{(d-1)}(T)\right\|>2^{N}\right\}\right) \leq c k^{-c \kappa} .
$$

If $\kappa$ is large enough, the right-hand terms form a converging series. As $m$ is invariant under the Zorich algorithm, we conclude that almost surely, the iterates $T^{(k)}$ of $T$ under the Zorich algorithm satisfy

$$
\left\|Z_{(d-1)}\left(T^{(k)}\right)\right\| \leq 2^{\kappa(\log k)^{d-1}},
$$

for all large $k$.

On the other hand, the exponential rate of growth of the $Q(k)$ (in the Zorich algorithm) is given by the largest Lyapunov exponent of the Teichmüller flow, which is positive. 
We conclude that there exists $\kappa_{1}$ such that almost all i.e.m. $T$ satisfy

$$
\log \left\|Z_{(d-1)}(k)\right\| \leq \kappa_{1}[\log \log \|Q(k)\|]^{d-1}
$$

for all large enough $k$.

Question. Does one have almost surely

$$
\left\|Z_{(d-1)}(k)\right\|=\mathrm{O}\left([\log \|Q(k)\|]^{C}\right)
$$

for some $C>0$ ?

4.8. Proof of the proposition. Let $\gamma, \mathcal{A}^{\prime}, D$ be as in the proposition. Let $T$ be an i.e.m. in $\Delta(\gamma)$ satisfying Keane's condition. Define, for $n \geq 0$,

$$
\begin{aligned}
Q^{\prime}(n, T) & =\sum_{\alpha \in \mathcal{A}^{\prime}} Q_{\alpha}(n, T), \\
Q_{e x t}(n, T) & =\sum_{\alpha \in \mathcal{A} \backslash \mathcal{A}^{\prime}} Q_{\alpha}(n, T),
\end{aligned}
$$

where $Q_{\alpha}(n, T)$ is the shorthand for $Q_{\alpha}\left(\left(\gamma_{j}(T)\right)_{0 \leq j \leq n}\right)$ (see the beginning of 4.6).

Lemma 1. If the names of the arrows $\gamma^{(m)}(T)$ belong to $\mathcal{A}^{\prime}$ for $m \leq n$, we have

$$
Q_{\text {ext }}(n, T) \leq(d-2) Q^{\prime}(n, T) \text {. }
$$

(Recall that, as $1<D<d$, we have $d \geq 3$.)

Proof. We start with $Q_{\alpha}(0, T)=1$ for all $\alpha \in \mathcal{A}$. Divide the segment $[1, n]$ into maximal 1-segments into which the name of the arrows is the same; let $\left[n_{i}, n_{i+1}\right)$ be such a segment, with arrows of name $\alpha_{i} \in \mathcal{A}^{\prime}$. The secondary names of these arrows appear with some periodicity $d_{i}<d$; moreover, if $n_{i}>1$, the secondary name of $\gamma^{\left(n_{i}\right)}$ is $\alpha_{i-1} \in \mathcal{A}^{\prime}$; if $n_{i}=1, i=0, n_{1}>d_{0}$, the secondary name of $\gamma^{(m)}$ is $\alpha_{1}$ for each $m=n_{1}-k d_{0}, k>0$. For $n_{i} \leq m<n_{i+1}$ we have

$$
\begin{aligned}
Q_{\text {ext }}(m, T) & =Q_{\text {ext }}(m-1, T), \\
Q^{\prime}(m, T) & =Q^{\prime}(m-1, T)+Q_{\alpha_{i}}\left(n_{i}-1, T\right)
\end{aligned}
$$

if the secondary name of $\gamma^{(m)}$ is in $\mathcal{A}^{\prime}$ and

$$
\begin{aligned}
Q_{e x t}(m, T) & =Q_{e x t}(m-1, T)+Q_{\alpha_{i}}\left(n_{i}-1, T\right), \\
Q^{\prime}(m, T) & =Q^{\prime}(m-1, T)
\end{aligned}
$$

otherwise. In each segment except perhaps the first one, the number of secondary names in $\mathcal{A} \backslash \mathcal{A}^{\prime}$ does not exceed $(d-2)$ times the number of secondary names in $\mathcal{A}^{\prime}$. In the first segment, we write $n_{1}=k d_{0}+n_{1}^{\prime}, 0<n_{1}^{\prime} \leq d_{0}$; again the number of secondary names in $\mathcal{A} \backslash \mathcal{A}^{\prime}$ does not exceed $(d-2)$ times the number of secondary names in $\mathcal{A}^{\prime}$ in the subsegment $\left[n_{1}^{\prime}, n_{1}\right)$. Finally we have for $0 \leq m<n_{1}^{\prime}$ that $Q^{\prime}(m, T) \geq D \geq 2, Q_{e x t}(m, T) \leq Q_{e x t}(0, T)+m \leq d-D+d_{0}-1 \leq 2 d-4$ and the estimate of the lemma follows.

Let $1 \leq D_{1} \leq D, n \geq 0, C_{1}>0$. We say that $T \in \Delta(\gamma)$ is $\left(D_{1}, n, C_{1}\right)$-balanced if we have

$$
Q_{\alpha}(n, T) \geq C_{1}^{-1} Q^{\prime}(n, T)
$$

for at least $D_{1}$ of the indices $\alpha \in \mathcal{A}^{\prime}$. The property only depends on the path $\left(\gamma^{(m)}(T)\right)_{0<m \leq n}$, and we will also say that this path is $\left(D_{1}, n, C_{1}\right)$-balanced. Clearly, any $T$ is $(1, n, D)$-balanced (for all $n \geq 0$ ). 
Lemma 2. Assume that $\gamma$ is $\left(D, N, C_{0}\right)$-balanced, for some constant $C_{0}>0$. Then we can find $\Delta^{\prime}(\gamma) \subset \Delta(\gamma)$ satisfying the conclusions of the proposition, with $l=l(d)$ and $\eta=\eta\left(d, C_{0}\right)$.

Proof. Let $\gamma^{\prime}=\left(\gamma^{(n)}\right)_{0<n \leq M}$ be an extension of $\gamma$ with minimal length such that the name $\alpha$ of $\gamma^{(M)}$ is not in $\mathcal{A}^{\prime}$. Then $M-N$ is bounded by the diameter of $\mathcal{D}$, i.e. in terms of $d$ only. Therefore there exists $C_{*}=C_{*}(d)$ such that $\gamma^{\prime}$ is $\left(D, M-1, C_{*} C_{0}\right)$-balanced; moreover, the path $\gamma^{\prime \prime}=\left(\gamma^{(n)}\right)_{0<n<M}$ satisfies

$$
\operatorname{vol}_{d-1} \Delta\left(\gamma^{\prime \prime}\right) \geq \eta^{\prime \prime} \operatorname{vol}_{d-1} \Delta(\gamma)
$$

with $\eta^{\prime \prime}=\eta^{\prime \prime}\left(C_{0}, d\right)$. Then, for all $\beta \in \mathcal{A}^{\prime}$, we have

$$
\begin{aligned}
Q_{\alpha}(M-1, T) & \leq Q_{\text {ext }}(M-1, T) \\
& \leq(d-2) Q^{\prime}(M-1, T) \\
& \leq(d-2) C_{*} C_{0} Q_{\beta}(M-1, T),
\end{aligned}
$$

and therefore

$$
\operatorname{vol}_{d-1} \Delta\left(\gamma^{\prime}\right) \geq \eta^{\prime} \operatorname{vol}_{d-1} \Delta\left(\gamma^{\prime \prime}\right)
$$

with $\eta^{\prime}=\left(1+(d-2) C_{*} C_{0}\right)^{-1}$. We take $\eta=\eta^{\prime} \eta^{\prime \prime}, \Delta^{\prime}(\gamma)=\Delta\left(\gamma^{\prime}\right)$. Finally $l$ is bounded because $M-N$ is bounded.

When $\gamma$ is only $(\tilde{D}, N, \tilde{C})$-balanced for some $\tilde{D}<D$, the strategy will be to extend $\gamma$ without losing volume in order to obtain a more balanced path; at the end we should be able to apply Lemma 2 (unless we have already found $\Delta^{\prime}(\gamma)$ ).

We therefore assume that $\gamma$ is $(\tilde{D}, N, \tilde{C})$-balanced. This is certainly satisfied with $\tilde{D}=1, \tilde{C}=D$. Denote by $\tilde{\mathcal{A}}$ the set of $\alpha$ such that

$$
Q_{\alpha}(N, T) \geq \tilde{C}^{-1} Q^{\prime}(N, T) .
$$

The first step is to extend $\gamma$ to a path $\gamma^{\prime}=\left(\gamma^{(n)}(T)\right)_{0<n \leq N^{\prime}}$ of minimal length such that the name of $\gamma^{\left(N^{\prime}\right)}$ is not in $\tilde{\mathcal{A}}$. When $N^{\prime}=N+1$, there might be two choices for $\gamma^{\left(N^{\prime}\right)}$ and we choose the one which gives the largest volume to $\Delta\left(\gamma^{\prime}\right)$.

In any case, an argument completely similar to the one in the proof of Lemma 2 leads to the estimate

$$
\operatorname{vol}_{d-1} \Delta\left(\gamma^{\prime}\right) \geq \eta^{\prime} \operatorname{vol}_{d-1} \Delta(\gamma)
$$

with a constant $\eta^{\prime}=\eta^{\prime}(d, \tilde{C})$.

If the name of $\gamma^{\left(N^{\prime}\right)}$ does not belong to $\mathcal{A}^{\prime}$, we can take as in Lemma $2, \Delta^{\prime}(\gamma)=$ $\Delta\left(\gamma^{\prime}\right)$ and the proof of the proposition is over. We now assume that the name of $\gamma^{\left(N^{\prime}\right)}$ belongs to $\mathcal{A}^{\prime} \backslash \tilde{\mathcal{A}}$.

The subset $\Delta^{\prime}(\gamma)$ of $\Delta(\gamma)$ we are looking for will be contained in $\Delta\left(\gamma^{\prime}\right)$. Observe that there exists $C_{*}=C_{*}(d)$ such that $\gamma^{\prime}$ is $\left(\tilde{D}, N^{\prime}, C_{*} \tilde{C}\right)$-balanced.

Case A. In the loop of arrows of the same name which starts with $\gamma^{\left(N^{\prime}\right)}$, no secondary name belongs to $\mathcal{A}^{\prime} \backslash \tilde{\mathcal{A}}$.

Let $\alpha$ be the name of $\gamma^{\left(N^{\prime}\right)}, \beta_{0}, \ldots, \beta_{r-1}$ being the successive secondary names in the loop. Letting $k>0$, we write $k=r l+m, 0 \leq m<r$. Let $\gamma_{1}(k)$ be the path extending $\gamma^{\prime}$ such that

- the name of $\gamma_{1}(k)^{(n)}$ is $\alpha$ for $N^{\prime}<n<N^{\prime}+k:=N_{1}(k)$;

- the name of $\gamma_{1}(k)^{\left(N_{1}(k)\right)}$ is $\beta_{m}$. 
Observe that it follows immediately from the definition of $R_{0}, R_{1}$ in 1.2.1 that the indices $\beta_{0}, \ldots, \beta_{r-1}$ are distinct. Therefore, we will have, for $0 \leq k_{1}=r l_{1}+m_{1}<k$ :

$$
\begin{aligned}
& Q_{\beta_{j}}\left(N^{\prime}+k_{1}, T\right)=Q_{\beta_{j}}\left(N^{\prime}-1, T\right)+ \begin{cases}l_{1} Q_{\alpha}\left(N^{\prime}-1, T\right) & \text { if } m_{1}<j \\
\left(l_{1}+1\right) Q_{\alpha}\left(N^{\prime}-1, T\right) & \text { if } m_{1} \geq j\end{cases} \\
& Q_{\alpha}\left(N^{\prime}+k_{1}, T\right)=Q_{\alpha}\left(N^{\prime}-1, T\right),
\end{aligned}
$$

and also

$$
\begin{aligned}
Q_{\beta_{j}}\left(N^{\prime}+k, T\right) & =Q_{\beta_{j}}\left(N^{\prime}+k-1, T\right), \\
Q_{\alpha}\left(N^{\prime}+k, T\right) & =Q_{\alpha}\left(N^{\prime}-1, T\right)+Q_{\beta_{m}}\left(N^{\prime}+k-1, T\right) .
\end{aligned}
$$

For any $k>0$, the extension from $\gamma$ to $\gamma_{1}(k)$ is covered by the same number of $(D-1)$-segments, which is bounded in terms of $d$ only.

For those $k$ such that $\beta_{m} \notin \mathcal{A}^{\prime}$, we include $\Delta\left(\gamma_{1}(k)\right)$ in $\Delta^{\prime}(\gamma)$.

The formulas for the volumes give

$$
\operatorname{vol}_{d-1}\left(\left[\bigcup_{0<k_{1}<k} \Delta\left(\gamma_{1}\left(k_{1}\right)\right)\right]^{c}\right)=\frac{\prod Q_{\beta_{j}}\left(N^{\prime}, T\right)}{\prod Q_{\beta_{j}}\left(N^{\prime}+k-1, T\right)} \operatorname{vol}_{d-1} \Delta\left(\gamma^{\prime}\right) .
$$

We keep for further consideration all $\gamma_{1}(k)$ with

$$
k Q_{\alpha}\left(N^{\prime}-1, T\right) \leq Q^{\prime}\left(N^{\prime}-1, T\right) .
$$

The formula above shows that together they will fill a definite proportion of $\Delta\left(\gamma^{\prime}\right)$.

We also see that when $\beta_{m} \in \tilde{\mathcal{A}}, \gamma_{1}(k)$ will be $\left(\tilde{D}+1, N_{1}(k), C_{1}\right)$-balanced, with $C_{1}$ depending only on $d$. For each such $\gamma_{1}(k)$, we either apply Lemma 2 (if $\tilde{D}+1=D)$ or repeat the discussion, with $\gamma_{1}(k)$ in the place of $\gamma$, from a better starting hypothesis.

Case B. The complement of case A.

For an i.e.m. in $\Delta\left(\gamma^{\prime}\right)$ satisfying Keane's condition, we consider the three mutually exclusive possibilities:

- $T$ is of type $I$ if there exists $N_{1} \geq N^{\prime}$ such that all arrows $\gamma^{(n)}(T), N^{\prime} \leq$ $n \leq N_{1}$, have names in $\mathcal{A}^{\prime} \backslash \tilde{\mathcal{A}}$, and we have

$$
\sum_{\alpha \in \mathcal{A}^{\prime} \backslash \tilde{\mathcal{A}}} Q_{\alpha}\left(N_{1}, T\right) \geq Q^{\prime}\left(N^{\prime}, T\right) .
$$

We take a minimal such $N_{1}$.

- $T$ is of type $I I$ (respectively of type III) if it is not of type I and the first name of an arrow $\gamma^{(n)}(T), n>N^{\prime}$, which does not belong to $\mathcal{A}^{\prime} \backslash \tilde{\mathcal{A}}$ belongs to $\tilde{\mathcal{A}}$ (resp. to $\mathcal{A} \backslash \mathcal{A}^{\prime}$ ).

We deal separately with the three types.

a) All $T$ of type III will be contained in $\Delta^{\prime}(\gamma)$; for such a $T, M$ is the first integer $>N^{\prime}$ for which the name does not belong to $\mathcal{A}^{\prime} \backslash \tilde{\mathcal{A}}$. Observe that the segment $\left(\gamma^{(n)}(T)\right)_{N^{\prime} \leq n<M}$ is a $(D-1)$-segment because card $\left(\mathcal{A}^{\prime} \backslash \tilde{\mathcal{A}}\right)<D$. As $N^{\prime}-N$ is bounded in terms of $d$ only, the number of $(D-1)$-segments needed to cover $\left(\gamma^{(n)}(T)\right)_{N \leq n<M}$ is bounded in terms of $d$ only.

b) Assume that $T$ is of type II. Let $N_{1}$ be the smallest integer $n>N^{\prime}$ such that the name of $\gamma^{(n)}(T)$ does not belong to $\mathcal{A}^{\prime} \backslash \tilde{\mathcal{A}}$; this name belongs to $\tilde{\mathcal{A}}$. Let $\gamma_{1}=\left(\gamma^{(n)}(T)\right)_{0<n \leq N_{1}}$. When $T$ varies among i.e.m.'s of type II, the $\gamma_{1}$ form an at 
most countable collection such that the corresponding simplices $\Delta\left(\gamma_{1}\right)$ have disjoint interiors (and are contained in $\Delta\left(\gamma^{\prime}\right)$ ). Every $T_{1}$ belonging to some $\Delta\left(\gamma_{1}\right)$ is also of type II. We claim that every $\gamma_{1}$ is $\left(D_{1}, N_{1}, C_{1}\right)$-balanced with $D_{1}>\tilde{D}$ and $C_{1}=C_{1}(\tilde{C}, d)$ (see the proof below). As for type III, the number of $(D-1)$ segments needed to cover $\gamma_{1}^{(n)}, N \leq n<N_{1}$, is bounded in terms of $d$ only.

c) Assume that $T$ is of type I. With $N_{1}$ minimal as in the definition of type I, take $\gamma_{1}=\left(\gamma^{(n)}(T)\right)_{0<n \leq N_{1}}$. When $T$ varies among i.e.m.'s of type I, the $\gamma_{1}$ form again an at most countable collection for which the corresponding simplices $\Delta\left(\gamma_{1}\right)$ have disjoint interiors (and are contained in $\Delta\left(\gamma^{\prime}\right)$ ). Every $T_{1}$ belonging to some $\Delta\left(\gamma_{1}\right)$ is also of type I. We claim that every $\gamma_{1}$ is $\left(D_{1}, N_{1}, C_{1}\right)$-balanced with $D_{1}>\tilde{D}$ and $C_{1}=C_{1}(\tilde{C}, d)$ (see the proof below). The number of $(D-1)$-segments needed to cover $\gamma_{1}^{(n)}, N \leq n<N_{1}$, is bounded in terms of $d$ only.

The discussion above leads in case B to a countable partition (up to a codimension one subset) of $\Delta\left(\gamma^{\prime}\right)$ into subsimplices of type III which will be included in $\Delta^{\prime}(\gamma)$ and simplices $\Delta\left(\gamma_{1}\right)$ (of type I or II) which satisfy the same hypotheses as $\Delta(\gamma)$ but are better balanced (i.e. $\left.D_{1}>\tilde{D}\right)$; when $D_{1}=D$, we can apply Lemma 2 to $\gamma_{1}$; when $D_{1}<D$, we repeat the discussion with $\gamma_{1}$ instead of $\gamma$. The process stops in less than $D$ steps and gives the conclusion of the proposition.

Proof of the claim for type II. As T is not of type I, we have

$$
\sum_{\alpha \in \mathcal{A}^{\prime} \backslash \tilde{\mathcal{A}}} Q_{\alpha}\left(N_{1}-1, T\right)<Q^{\prime}\left(N^{\prime}, T\right) .
$$

Let us consider a maximal 1-segment contained in $\left(\gamma^{(n)}(T)\right)_{N^{\prime} \leq n<N_{1}}$. As we are not in case $\mathrm{A}$, there is a definite proportion, depending only on $\bar{d}$, of secondary names which belong to $\mathcal{A}^{\prime} \backslash \tilde{\mathcal{A}}$. This implies that we must have

$$
Q^{\prime}\left(N_{1}-1, T\right) \leq C_{1}^{\prime} Q^{\prime}\left(N^{\prime}, T\right),
$$

with $C_{1}^{\prime}$ depending only on $d$. On the other hand, if $\alpha \in \mathcal{A}^{\prime} \backslash \tilde{\mathcal{A}}$ and $\beta \in \tilde{\mathcal{A}}$ are the names of $\gamma^{\left(N_{1}-1\right)}(T), \gamma^{\left(N_{1}\right)}(T)$ respectively, we have

$$
\begin{aligned}
Q_{\alpha}\left(N_{1}, T\right) & =Q_{\alpha}\left(N_{1}-1, T\right)+Q_{\beta}\left(N_{1}-1, T\right) \\
& \geq\left(C_{*} \tilde{C}\right)^{-1} Q^{\prime}\left(N^{\prime}, T\right) .
\end{aligned}
$$

It follows that $\gamma_{1}$ is $\left(\tilde{D}+1, N_{1}, C_{1}\right)$-balanced with $C_{1}=C_{1}^{\prime} C_{*} \tilde{C}$.

Proof of the claim for type I. By definition of $N_{1}$, we have again

$$
\sum_{\alpha \in \mathcal{A}^{\prime} \backslash \tilde{\mathcal{A}}} Q_{\alpha}\left(N_{1}-1, T\right)<Q^{\prime}\left(N^{\prime}, T\right)
$$

and it follows again that

$$
Q^{\prime}\left(N_{1}-1, T\right) \leq C_{1}^{\prime} Q^{\prime}\left(N^{\prime}, T\right) .
$$

By definition of $N_{1}$, we have now

$$
\sum_{\alpha \in \mathcal{A}^{\prime} \backslash \tilde{\mathcal{A}}} Q_{\alpha}\left(N_{1}, T\right) \geq Q^{\prime}\left(N^{\prime}, T\right),
$$

and it follows that $\gamma_{1}$ is $\left(\tilde{D}+1, N_{1}, C_{1}\right)$-balanced with $C_{1}$ depending only on $\tilde{C}$ and $d$. 
The proof of the proposition, and therefore also of the full measure statement, is now complete.

Appendix A. Roth-type conditions in a concrete family of interval exchange maps.

A.1. Let $\mathcal{A}=(A, B, C, D)$. The Rauzy diagram of the pair

$$
\left(\pi_{0}, \pi_{1}\right)=\left(\begin{array}{cccc}
A & B & C & D \\
D & C & B & A
\end{array}\right)
$$

is indicated in 1.2.2. The suspension of an i.e.m. with these combinatorial data leads to a holomorphic 1-form with a double zero on a genus two surface.

In this diagram, we define for $n \geq 0$ a loop $\gamma(n)$ based at $\left(\pi_{0}, \pi_{1}\right)$ by asking that the names of the successive arrows should be $D^{2} C D A^{2} B^{n} A$. The product of the $V$ matrices around this loop is

$$
M(n)=\left(\begin{array}{cccc}
1 & 1 & 1 & 1 \\
n & n+1 & 0 & 0 \\
0 & 0 & 2 & 1 \\
n+1 & n+2 & 2 & 2
\end{array}\right)
$$

with characteristic polynomial

$$
\chi_{n}(X)=X^{4}-(n+6) X^{3}+(3 n+10) X^{2}-(n+6) X+1 .
$$

Setting $U=X+X^{-1}$ leads to

$$
\chi_{n}(X)=X^{2}\left(U^{2}-(n+6) U+3 n+8\right) .
$$

The eigenvalues of $M(n)$ are thus given by

$$
\lambda+\lambda^{-1}=U^{ \pm}:=\frac{1}{2}\left(n+6 \pm \sqrt{n^{2}+4}\right) .
$$

The case $n=0$ is degenerate, with $U^{+}=4, U^{-}=2$. When $n>0$, both $U^{+}, U^{-}$ are $>2$; we will denote the eigenvalues by $\lambda_{u}^{+}>\lambda_{u}^{-}(>1)>\lambda_{s}^{-}>\lambda_{s}^{+}$, and denote by $e_{u}^{+}, e_{u}^{-}, e_{s}^{-}, e_{s}^{+}$the corresponding eigenvectors of the transposed matrix ${ }^{t} M(n)$.

The eigenvector associated to the eigenvalue $\lambda$ is proportional to

$$
\left((\lambda-1)\left(\lambda^{2}-4 \lambda+2\right), \lambda^{3}-4 \lambda^{2}+3 \lambda-1, \lambda(\lambda-1),(\lambda-1)^{2}\right) .
$$

A.2. As $n \rightarrow+\infty$, one has

$$
\begin{aligned}
\lim U^{+}-(n+3) & =\lim \lambda_{u}^{+}-(n+3)=0, \\
\lim U^{-} & =3, \lim \lambda_{u}^{-}=G:=\frac{\sqrt{5}+3}{2} .
\end{aligned}
$$

One can also choose eigenvectors to obtain:

$$
\begin{aligned}
& \lim e_{u}^{+}=E_{u}^{+}:=(1,1,0,0), \\
& \lim e_{u}^{-}=E_{u}^{-}:=(-1,-1, G-1,1), \\
& \lim e_{s}^{-}=E_{s}^{-}:=\left(-1,-1, G^{-1}-1,1\right), \\
& \lim e_{s}^{+}=E_{s}^{+}:=(2,1,0,-1) .
\end{aligned}
$$


These four limit vectors form a basis of $\mathbb{R}^{4}$ in which we rewrite ${ }^{t} M(n)$ :

$$
\begin{aligned}
& { }^{t} M(n) E_{u}^{+}=(n+3) E_{u}^{+}-E_{s}^{+}+\frac{1}{\sqrt{5}}\left(E_{u}^{-}-E_{s}^{-}\right), \\
& { }^{t} M(n) E_{s}^{+}=E_{u}^{+}, \\
& { }^{t} M(n) E_{u}^{-}=G\left(E_{u}^{+}+E_{u}^{-}\right), \\
& { }^{t} M(n) E_{s}^{-}=G^{-1}\left(E_{u}^{+}+E_{s}^{-}\right) .
\end{aligned}
$$

For the corresponding coordinates, this gives

$$
\begin{aligned}
& X_{u}^{+}=(n+3) x_{u}^{+}+x_{s}^{+}+G x_{u}^{-}+G^{-1} x_{s}^{-}, \\
& X_{s}^{+}=-x_{u}^{+}, \\
& X_{u}^{-}=\frac{1}{\sqrt{5}} x_{u}^{+}+G x_{u}^{-}, \\
& X_{s}^{-}=-\frac{1}{\sqrt{5}} x_{u}^{+}+G^{-1} x_{u}^{-} .
\end{aligned}
$$

A.3. The following two lemmas express that for $n \geq 4$ certain cone conditions are satisfied.

Lemma 1. For $n \geq 4, x_{u}^{+} \geq \operatorname{Max}\left(\left|x_{s}^{+}\right|,\left|x_{u}^{-}\right|,\left|x_{s}^{-}\right|\right)$one has

$$
X_{u}^{+} \geq \operatorname{Max}\left((n-1)\left|X_{s}^{+}\right|,(n-1)\left|X_{s}^{-}\right|, \frac{10-3 \sqrt{5}}{3}\left|X_{u}^{-}\right|\right)
$$

and

$$
X_{u}^{+} \geq(n-1) x_{u}^{+} .
$$

Proof. As $G^{-1}+\frac{1}{\sqrt{5}}<1$ and $G+G^{-1}=3$, we have

$$
\operatorname{Max}\left((n-1)\left|X_{s}^{+}\right|,(n-1)\left|X_{s}^{-}\right|,(n-1)\left|x_{u}^{+}\right|\right) \leq X_{u}^{+} .
$$

If $x_{u}^{-} \geq 0$, one has

$$
X_{u}^{+} \geq(n+1) x_{u}^{+}+G x_{u}^{-} \geq 2 X_{u}^{-}
$$

because $n+1-2 / \sqrt{5}>G$ for $n \geq 4$.

For $x_{u}^{-}<0$, one has $X_{u}^{+} \geq(n+1) x_{u}^{+}-G\left|x_{u}^{-}\right|$. On the one hand,

$$
(n+1) x_{u}^{+}-G\left|x_{u}^{-}\right| \geq(n+1) \sqrt{5} X_{u}^{-} ;
$$

on the other hand,

$$
(n+1) x_{u}^{+}-G\left|x_{u}^{-}\right| \geq-\gamma X_{u}^{-}
$$

as soon as $n+1+\gamma / \sqrt{5} \geq(\gamma+1) G$, which allows us to take $\gamma=\frac{10-3 \sqrt{5}}{3}$ for $n \geq 4$.

Lemma 2. For $n \geq 4, \operatorname{Max}\left(\left|x_{u}^{+}\right|,\left|x_{u}^{-}\right|\right) \geq \operatorname{Max}\left(\left|x_{s}^{+}\right|,\left|x_{s}^{-}\right|\right)$, one has

$$
\begin{aligned}
& \operatorname{Max}\left(\left|X_{u}^{+}\right|,\left|X_{u}^{-}\right|\right) \geq\left(G-\frac{1}{\sqrt{5}}\right) \operatorname{Max}\left(\left|X_{s}^{+}\right|,\left|X_{s}^{-}\right|\right), \\
& \operatorname{Max}\left(\left|X_{u}^{+}\right|,\left|X_{u}^{-}\right|\right) \geq\left(G-\frac{1}{\sqrt{5}}\right) \operatorname{Max}\left(\left|x_{u}^{+}\right|,\left|x_{u}^{-}\right|\right) .
\end{aligned}
$$


Proof. When $\left|x_{u}^{+}\right| \geq\left|x_{u}^{-}\right|$, this follows from Lemma 1. If $0 \leq\left|x_{u}^{+}\right| \leq x_{u}^{-}$, one has

$$
X_{u}^{-} \geq\left(G-\frac{1}{\sqrt{5}}\right) x_{u}^{-} \geq\left(G-\frac{1}{\sqrt{5}}\right) \operatorname{Max}\left(\left|X_{s}^{+}\right|,\left|X_{s}^{-}\right|\right)
$$

because $G^{-1}+\frac{1}{\sqrt{5}}<1$. 1.

One should observe in Lemma 1 that $\frac{10-3 \sqrt{5}}{3}>1$ and in Lemma 2 that $G-\frac{1}{\sqrt{5}}>$

Lemma 3. Equip $\mathbb{R}^{\mathcal{A}}$ with the sup norm. Then, for any integers $n_{1}, \ldots, n_{k}>0$, we have

$$
\prod_{i=1}^{k}\left(n_{i}+1\right) \leq\left\|^{t} M\left(n_{k}\right) \cdots^{t} M\left(n_{1}\right)\right\|_{\infty} \leq \prod_{i=1}^{k}\left(2 n_{i}+4\right) .
$$

Proof. The upper bound follows from $\left\|^{t} M(n)\right\| \leq 2 n+4$ for $n>0$, the lower bound from the fact that

$$
{ }^{t} M(n)(1,1,0,0)-(n+1)(1,1,0,0)
$$

is a nonnegative vector.

A.4. Let $\Sigma$ be the set of sequences $\left(n_{i}\right)_{i>0}$ of integers $\geq 4$. To each sequence in $\Sigma$ we associate the infinite path $\gamma\left(n_{1}\right) \gamma\left(n_{2}\right) \cdots$ starting at $\left(\pi_{0}, \pi_{1}\right)$. The cone property of Lemma 1 guarantees that there is exactly one i.e.m. satisfying Keane's condition associated with this path. On the space $\Gamma$ of functions constant on each $j_{0}\left(I_{\alpha}\right)$, we have a complete filtration: the space $\Gamma_{s}$ has dimension 2 according to Lemma 2, contains the line $\mathbb{R} \delta$ (where $\delta$ is the displacement vector) and is contained in the hyperplane $\Gamma_{*}$ of zero mean.

Therefore conditions (b) and (c) in 1.3.2, 1.3.3 are automatically satisfied. Condition (a) is equivalent, in view of Lemma 3 , to

$$
\log n_{k}=\mathrm{o}\left(\sum_{i<k} \log n_{i}\right) .
$$

\section{Appendix B. A nonuniquely ergodic interval exchange map satisfying condition (a).}

B.1. Let $m, n, p$ be nonnegative integers. In the Rauzy diagram of the pair $\left(\pi_{0}, \pi_{1}\right)$ $=\left(\begin{array}{llll}A & B & C & D \\ D & C & B & A\end{array}\right)$ (cf. 1.2.2), consider the loop $\gamma_{0}(m, n, p)$ based at $\left(\pi_{0}, \pi_{1}\right)$ such that the names of the successive arrows are

$$
D^{3 m+1} B C^{n} B D C^{p} D .
$$

We also consider the dual loop $\gamma_{1}(m, n, p)$ which is deduced from $\gamma_{0}(m, n, p)$ by 
means of the canonical involution and whose arrows have names

$$
A^{3 m+1} C B^{n} C A B^{p} A \text {. }
$$

Given three sequences $\left(m_{k}\right)_{k \geq 0},\left(n_{k}\right)_{k \geq 0}$ and $\left(p_{k}\right)_{k \geq 0}$ we also consider the infinite path $\Gamma$, based at $\left(\begin{array}{llll}A & B & C & D \\ D & C & B & A\end{array}\right)$, which is obtained by composing

$$
\gamma_{0}\left(m_{0}, n_{0}, p_{0}\right) \gamma_{1}\left(m_{1}, n_{1}, p_{1}\right) \cdots \gamma_{0}\left(m_{2 k}, n_{2 k}, p_{2 k}\right) \gamma_{1}\left(m_{2 k+1}, n_{2 k+1}, p_{2 k+1}\right) \cdots
$$

The matrix $Z_{0}(m, n, p)$ associated to $\gamma_{0}(m, n, p)$ is

$$
\left(\begin{array}{cccc}
1 & 0 & 0 & 0 \\
0 & 2 & p+2 & p+1 \\
0 & n & (n+1)(p+1) & p(n+1) \\
m+1 & m(n+2)+1 & m(n+2)(p+1)+m+1 & p m(n+2)+m+1
\end{array}\right)
$$

where the vectors of the canonical basis of $\mathbb{R}^{\mathcal{A}}$ are ordered alphabetically. Analogously the matrix $Z_{1}(m, n, p)$ associated to $\gamma_{1}(m, n, p)$ is

$$
\left(\begin{array}{cccc}
p m(n+2)+m+1 & m(n+2)(p+1)+m+1 & m(n+2)+1 & m+1 \\
p(n+1) & (n+1)(p+1) & n & 0 \\
p+1 & p+2 & 2 & 0 \\
0 & 0 & 0 & 1
\end{array}\right)
$$

We set

$$
Q(k)=Z_{0}\left(m_{0}, n_{0}, p_{0}\right) Z_{1}\left(m_{1}, n_{1}, p_{1}\right) \cdots Z_{\varepsilon}\left(m_{k-1}, n_{k-1}, p_{k-1}\right)
$$

with $k-1 \equiv \varepsilon \bmod 2$. We denote by $e_{A}(k), e_{B}(k), e_{C}(k), e_{D}(k)$ the column vectors of $Q(k)$.

B.2. Let $m_{0}=0$ and choose $n_{0} \gg 1$. The integer $p_{0}$ will be chosen later but it will be such that $p_{0} \geq n_{0}$. One has

$$
\begin{aligned}
& e_{A}(1)={ }^{t}\left(\begin{array}{llll}
1 & 0 & 0 & 1
\end{array}\right), \\
& e_{B}(1)=n_{0}\left[^{t}\left(\begin{array}{llll}
0 & 0 & 1 & 0
\end{array}\right)+\mathrm{O}\left(n_{0}^{-1}\right)\right] \\
& e_{C}(1)=n_{0} p_{0}\left[{ }^{t}\left(\begin{array}{llll}
0 & 0 & 1 & 0
\end{array}\right)+\mathrm{O}\left(n_{0}^{-1}\right)\right], \\
& e_{D}(1)=n_{0} p_{0}\left[{ }^{t}\left(\begin{array}{llll}
0 & 0 & 1 & 0
\end{array}\right)+\mathrm{O}\left(n_{0}^{-1}\right)\right] .
\end{aligned}
$$


We determine then $m_{1}, p_{0}, n_{1}, m_{2}, \ldots, m_{k}, p_{k-1}, n_{k}, m_{k+1}, p_{k}, \ldots$ through the following formulas:

$$
\begin{aligned}
\Pi_{0} & :=n_{0}, \\
\Pi_{1} & :=m_{1} \Pi_{0}^{-1}=n_{0}^{2}, \\
\Pi_{2} & :=p_{0} \Pi_{1}^{-1}=\left(n_{0}+1\right)^{2}, \\
& \vdots \\
\Pi_{3 l} & :=n_{l} \Pi_{3 l-1}^{-1}=\left(n_{0}+3 l-1\right)^{2}, \\
\Pi_{3 l+1} & :=m_{l+1} \Pi_{3 l}^{-1}=\left(n_{0}+3 l\right)^{2}, \\
\Pi_{3 l+2} & :=p_{l} \Pi_{3 l+1}^{-1}=\left(n_{0}+3 l+1\right)^{2}, \\
& \vdots \\
m_{1} & :=n_{0}^{3}, \\
p_{0} & :=\left(n_{0}+1\right)^{2} \Pi_{1}=n_{0}^{2}\left(n_{0}+1\right)^{2}, \\
n_{1} & :=\left(n_{0}+2\right)^{2} \Pi_{2}=\left(n_{0}+1\right)^{2}\left(n_{0}+2\right)^{2}, \\
\vdots & \\
n_{l+1} & :=\left(n_{0}+3 l+2\right)^{2} \Pi_{3 l+2}, \\
p_{l} & :=\left(n_{0}+3 l\right)^{2} \Pi_{3 l}, \\
&
\end{aligned}
$$

Thus one has, for $l \geq 0$,

$$
\begin{aligned}
p_{l} & =\left(n_{0}+3 l\right)^{2}\left(n_{0}+3 l+1\right)^{2}, \\
n_{l+1} & =\left(n_{0}+3 l+1\right)^{2}\left(n_{0}+3 l+2\right)^{2}, \\
m_{l+2} & =\left(n_{0}+3 l+2\right)^{2}\left(n_{0}+3 l+3\right)^{2},
\end{aligned}
$$

and also $m_{1}=n_{0}^{3}$. For all $k \geq-1$ we set

$$
c_{k}=n_{0}^{3}\left[\frac{\left(n_{0}+k\right) !}{n_{0} !}\right]^{2}
$$


so that one has $n_{0}=c_{-1}$ and

$$
\begin{aligned}
c_{1} & =n_{0} p_{0}, \\
c_{2} & =m_{1} n_{1}, \\
c_{4} & =m_{1} n_{1} p_{1}, \\
c_{5} & =m_{2} n_{2} n_{0} p_{0}, \\
c_{7} & =m_{2} n_{2} p_{2} n_{0} p_{0}, \\
c_{8} & =m_{3} n_{3} m_{1} n_{1} p_{1}, \\
\vdots & \\
c_{10} & =m_{3} n_{3} p_{3} m_{1} n_{1} p_{1},
\end{aligned}
$$

Let us check by induction that, setting $c_{-2}=1$, one has for $l \geq 0$ :

$$
\begin{aligned}
& e_{A}(2 l-1)=c_{6 l-8}\left[{ }^{t}\left(\begin{array}{llll}
1 & 0 & 0 & 1
\end{array}\right)+\mathrm{O}\left(n_{0}^{-1}\right)\right], \\
& e_{B}(2 l-1)=c_{6 l-7}\left[{ }^{t}\left(\begin{array}{llll}
0 & 0 & 1 & 0
\end{array}\right)+\mathrm{O}\left(n_{0}^{-1}\right)\right] \text {, } \\
& e_{C}(2 l-1)=c_{6 l-5}\left[{ }^{t}\left(\begin{array}{llll}
0 & 0 & 1 & 0
\end{array}\right)+\mathrm{O}\left(n_{0}^{-1}\right)\right] \text {, } \\
& e_{D}(2 l-1)=c_{6 l-5}\left[{ }^{t}\left(\begin{array}{llll}
0 & 0 & 1 & 0
\end{array}\right)+\mathrm{O}\left(n_{0}^{-1}\right)\right] \text {, } \\
& e_{D}(2 l)=c_{6 l-5}\left[{ }^{t}\left(\begin{array}{llll}
0 & 0 & 1 & 0
\end{array}\right)+\mathrm{O}\left(n_{0}^{-1}\right)\right], \\
& e_{C}(2 l)=c_{6 l-4}\left[{ }^{t}\left(\begin{array}{llll}
1 & 0 & 0 & 1
\end{array}\right)+\mathrm{O}\left(n_{0}^{-1}\right)\right] \text {, } \\
& e_{B}(2 l)=c_{6 l-2}\left[{ }^{t}\left(\begin{array}{llll}
1 & 0 & 0 & 1
\end{array}\right)+\mathrm{O}\left(n_{0}^{-1}\right)\right] \text {, } \\
& e_{A}(2 l)=c_{6 l-2}\left[{ }^{t}\left(\begin{array}{llll}
1 & 0 & 0 & 1
\end{array}\right)+\mathrm{O}\left(n_{0}^{-1}\right)\right] .
\end{aligned}
$$

We have already checked the first four relations for $l=1$. Assume that the first four relations are verified for a given value of $l$. Then

$$
e_{D}(2 l)=e_{D}(2 l-1)+\left(m_{2 l-1}+1\right) e_{A}(2 l-1),
$$

with

$$
m_{2 l-1} c_{6 l-8}=c_{6 l-6}=c_{6 l-5}\left(n_{0}+6 l-5\right)^{-2} .
$$

Moreover

$$
e_{C}(2 l)=2 e_{C}(2 l-1)+n_{2 l-1} e_{B}(2 l-1)+\left[m_{2 l-1}\left(n_{2 l-1}+2\right)+1\right] e_{A}(2 l-1),
$$

with

$$
\begin{aligned}
m_{2 l-1} n_{2 l-1} c_{6 l-8} & =c_{6 l-4}, \\
n_{2 l-1} c_{6 l-7} & =\left(n_{0}+6 l-6\right)^{-2} c_{6 l-4}, \\
2 c_{6 l-5} & =2\left(n_{0}+6 l-4\right)^{-2} c_{6 l-4}, \\
\left(2 m_{2 l-1}+1\right) c_{6 l-8} & =\mathrm{O}\left(\left(n_{0}+6 l\right)^{-2}\right) c_{6 l-4}
\end{aligned}
$$

and

$$
\begin{aligned}
e_{B}(2 l)= & \left(p_{2 l-1}+2\right) e_{C}(2 l-1)+\left(n_{2 l-1}+1\right)\left(p_{2 l-1}+1\right) e_{B}(2 l-1) \\
& +\left(m_{2 l-1}\left(n_{2 l-1}+2\right)\left(p_{2 l-1}+1\right)+m_{2 l-1}+1\right) e_{A}(2 l-1)
\end{aligned}
$$


with

$$
\begin{aligned}
\left(p_{2 l-1}+2\right) c_{6 l-5} & =\mathrm{O}\left(\left(n_{0}+6 l\right)^{-2} c_{6 l-2}\right), \\
\left(n_{2 l-1}+1\right)\left(p_{2 l-1}+1\right) c_{6 l-7} & =\mathrm{O}\left(\left(n_{0}+6 l\right)^{-2} c_{6 l-2}\right), \\
{\left[1+m_{2 l-1}\left(n_{2 l-1}+2 p_{2 l-1}+1\right)\right] c_{6 l-8} } & =\mathrm{O}\left(\left(n_{0}+6 l\right)^{-4} c_{6 l-2}\right), \\
m_{2 l-1} n_{2 l-1} p_{2 l-1} c_{6 l-8} & =c_{6 l-2} .
\end{aligned}
$$

The formula for $e_{A}(2 l)$ is completely similar.

Since one has

$$
\prod_{k \geq 0}\left[1+\mathrm{O}\left(\left(n_{0}+k\right)^{-2}\right)\right]-1=\mathrm{O}\left(n_{0}^{-1}\right)
$$

one gets the four last relations. Taking into account the canonical involution one can analogously obtain the first four relations.

B.3. The decomposition of the infinite path $\Gamma$ into loops $\gamma_{0}\left(m_{2 k}, n_{2 k}, p_{2 k}\right)$ and $\gamma_{1}\left(m_{2 k+1}, n_{2 k+1}, p_{2 k+1}\right)$ is nothing else than the decomposition for the accelerated Zorich algorithm. One has

$$
\left\|Z_{\varepsilon}\left(m_{k}, n_{k}, p_{k}\right)\right\| \sim\left(n_{0}+3 k\right)^{12}
$$

(with $\varepsilon \equiv k \bmod 2$ ), and

$$
\|Q(k)\| \sim c_{3 k-2}=n_{0}^{3}\left[\frac{\left(n_{0}+3 k-2\right) !}{n_{0} !}\right]^{2} .
$$

Thus one obtains

$$
\left\|Z_{\varepsilon}\left(m_{k}, n_{k}, p_{k}\right)\right\|=\mathrm{o}\left([\log \|Q(k)\|]^{12}\right)
$$

and the first condition in the definition of the Roth-type interval exchange map is (by far) satisfied.

B.4. From the formula and estimates of Section A.2.2 one gets

$$
\begin{aligned}
& \frac{e_{D}(2 l)}{\left\|e_{D}(2 l)\right\|_{1}}=\frac{e_{D}(2 l-1)}{\left\|e_{D}(2 l-1)\right\|_{1}}+\mathrm{O}\left(\left(n_{0}+6 l\right)^{-2}\right), \\
& \frac{e_{C}(2 l)}{\left\|e_{C}(2 l)\right\|_{1}}=\frac{e_{A}(2 l-1)}{\left\|e_{A}(2 l-1)\right\|_{1}}+\mathrm{O}\left(\left(n_{0}+6 l\right)^{-2}\right), \\
& \frac{e_{B}(2 l)}{\left\|e_{B}(2 l)\right\|_{1}}=\frac{e_{A}(2 l-1)}{\left\|e_{A}(2 l-1)\right\|_{1}}+\mathrm{O}\left(\left(n_{0}+6 l\right)^{-2}\right), \\
& \frac{e_{A}(2 l)}{\left\|e_{A}(2 l)\right\|_{1}}=\frac{e_{A}(2 l-1)}{\left\|e_{A}(2 l-1)\right\|_{1}}+\mathrm{O}\left(\left(n_{0}+6 l\right)^{-2}\right),
\end{aligned}
$$

and by applying the canonical involution one obtains similar formulas at the order $2 l+1$. Therefore one can conclude that if there exist two vectors $u_{A}$ and $u_{D}$ in $\mathbb{R}^{\mathcal{A}}$ 
such that $\left\|u_{A}\right\|=\left\|u_{D}\right\|=1$, then

$$
\begin{aligned}
& u_{A}=\left(\begin{array}{c}
1 / 2 \\
0 \\
0 \\
1 / 2
\end{array}\right)+\mathrm{O}\left(n_{0}^{-1}\right), \\
& u_{D}=\left(\begin{array}{c}
0 \\
0 \\
1 \\
0
\end{array}\right)+\mathrm{O}\left(n_{0}^{-1}\right), \\
& \lim _{l \rightarrow+\infty} \frac{e_{A}(l)}{\left\|e_{A}(l)\right\|_{1}}=\lim _{l \rightarrow+\infty} \frac{e_{B}(2 l)}{\left\|e_{B}(2 l)\right\|_{1}}=\lim _{l \rightarrow+\infty} \frac{e_{C}(2 l)}{\left\|e_{C}(2 l)\right\|_{1}}=u_{A}, \\
& \lim _{l \rightarrow+\infty} \frac{e_{D}(l)}{\left\|e_{D}(l)\right\|_{1}}=\lim _{l \rightarrow+\infty} \frac{e_{C}(2 l+1)}{\left\|e_{C}(2 l+1)\right\|_{1}}=\lim _{l \rightarrow+\infty} \frac{e_{B}(2 l+1)}{\left\|e_{B}(2 l+1)\right\|_{1}}=u_{D} .
\end{aligned}
$$

It is now easy to see that each point $u$ of the segment $\left[u_{A}, u_{D}\right] \subset\left(\mathbb{R}^{+}\right)^{\mathcal{A}}$ is the lengths datum for an interval exchange map with combinatorial datum $\left(\begin{array}{cccc}A & B & C & D \\ D & C & B & A\end{array}\right)$, satisfying Keane's condition and which is not uniquely ergodic: the interval exchange maps of this one-parameter family are topologically conjugate.

\section{ACKNOWLEDGEMENTS}

We are grateful to G. Forni for many stimulating discussions. This research has been supported by the following institutions: CNR, CNRS, MURST, INDAM, the French-Italian University, the Collège de France and the Scuola Normale Superiore. We are also grateful to the two former institutions and to the Centro di Ricerca Matematica "Ennio De Giorgi" in Pisa for hospitality.

\section{REFERENCES}

[Ar] P. Arnoux "Ergodicité générique des billiards polygonaux [d'après Kerckhoff, Masur, Smillie]" Séminaire Bourbaki n. 696, Astérisque 161-162 (1988) 203-221. MR0992210 (90c:58097)

[Bo] M. Boshernitzan "A condition for minimal interval exchange maps to be uniquely ergodic" Duke Math. J. 52 (1985) 723-752. MR0808101 (87i:28012)

[Ch] Y. Cheung "Hausdorff dimension of the set of nonergodic directions. With an appendix by M. Boshernitzan" Ann. of Math. 158 (2003) 661-678. MR2018932 (2004k:37069)

[Co] J. Coffey "Some remarks concerning an example of a minimal, non-uniquely ergodic interval exchange transformation" Math. Z. 199 (1988) 577-580. MR0968323 (90c:28025)

[FLP] A. Fathi, F. Laudenbach and V. Poenaru "Travaux de Thurston sur les surfaces" Astérisque 66-67 (1979). MR0568308 (82m:57003)

[Fo1] G. Forni "Solutions of the cohomological equation for area-preserving flows on compact surfaces of higher genus" Annals of Mathematics 146 (1997) 295-344. MR1477760 (99d:58102)

[Fo2] G. Forni "Deviation of ergodic averages for area-preserving flows on surfaces of higher genus" Annals of Mathematics 155 (2002) 1-103. MR1888794 (2003g:37009)

[Fo3] G. Forni, private communication (2003).

[GH] W.H. Gottschalk and G.A. Hedlund "Topological dynamics" American Mathematical Society Colloquium Publications, 36. American Mathematical Society, Providence, RI (1955). MR0074810(17:650e)

$[\mathrm{KH}]$ A. Katok and B. Hasselblatt "Introduction to the modern theory of dynamical systems" Encyclopedia of Mathematics and its Applications 54, Cambridge University Press, (1995). MR1326374(96c:58055) 
[KS] A. Katok and A.M. Stepin "Approximations in Ergodic Theory" Russ. Math. Surv. 22 (1967) 77-102. MR0219697 (36:2776)

[Ke1] M. Keane "Interval exchange transformations" Math. Z. 141 (1975) 25-31. MR0357739 $(50: 10207)$

[Ke2] M. Keane "Non-ergodic interval exchange transformations" Isr. J. Math. 26 (1977) 188196. MR0435353 (55:8313)

[Ker] S. P. Kerckhoff "Simplicial systems for interval exchange maps and measured foliations" Ergod. Th. Dynam. Sys. 5 (1985) 257-271. MR0796753 (87g:58075)

[KMS] S. Kerckhoff, H. Masur and J. Smillie "Ergodicity of billiard flows and quadratic differentials" Ann. of Math. 124 (1986) 293-311. MR0855297 (88f:58122)

[KN] H. B. Keynes and D. Newton "A "Minimal", Non-uniquely Ergodic Interval Exchange Transformation" Math. Z. 148 (1976) 101-105. MR0409766 (53:13518)

[KR] M. Keane and G. Rauzy "Stricte ergodicité des échanges d'intervalles" Math. Z. 174 (1980) 203-212. MR0593819 (82d:28014)

[KZ] M. Kontsevich and A. Zorich "Connected components of the moduli spaces of Abelian differentials with prescribed singularities" Inv. Math. 153 (2003) 631-678. MR2000471 (2005b:32030)

[Ma] H. Masur "Interval exchange transformations and measured foliations" Annals of Mathematics 115 (1982) 169-200. MR0644018 (83e:28012)

[MMY] S. Marmi, P. Moussa and J.-C. Yoccoz "On the cohomological equation for interval exchange maps" C. R. Math. Acad. Sci. Paris 336 (2003) 941-948. MR1994599 (2004i:37003)

[MS] H. Masur and J. Smillie "Quadratic differentials with prescribed singularities and pseudo-Anosov diffeomorphisms" Comment. Math. Helv. 68 (1993) 289-307. MR1214233 (94d:32028)

[Ra] G. Rauzy "Échanges d'intervalles et transformations induites" Acta Arith. (1979) 315328. MR0543205 (82m:10076)

[Re] M. Rees "An alternative approach to the ergodic theory of measured foliations" Ergod. Th. Dyn. Sys. 1 (1981) 461-488. MR0662738 (84c:58065)

[Ta] S. Tabachnikov "Billiards" Panoramas et Synthèses, S.M.F. 1 (1995). MR.1328336 (96c:58134)

[V1] W. Veech "Interval exchange transformations" Journal d'Analyse Mathématique 33 (1978) 222-272. MR0516048(80e:28034)

[V2] W. Veech "Gauss measures for transformations on the space of interval exchange maps" Ann. of Math. 115 (1982) 201-242. MR0644019 (83g:28036b)

[V3] W. Veech "The metric theory of interval exchange transformations I. Generic spectral properties" Amer. J. of Math. 106 (1984) 1331-1359. MR0765582 (87j:28024a)

[V4] W. Veech "The metric theory of interval exchange transformations II. Approximation by primitive interval exchanges" Amer. J. of Math. 106 (1984) 1361-1387. MR0765583 (87j:28024b)

[V5] W. Veech "The metric theory of interval exchange transformations III. The Sah Arnoux Fathi invariant" Amer. J. of Math. 106 (1984) 1389-1421. MR0765584 (87j:28024c)

[V6] W. Veech "The Teichmüller geodesic flow" Ann. of Math. 124 (1986) 441-530. MR0866707 (88g:58153)

[V7] W. Veech "Moduli spaces of quadratic differentials" Journal d'Analyse Mathématique 55 (1990) 117-171. MR1094714 (92e:32014)

$[\mathrm{Y}] \quad$ J.-C. Yoccoz "Continued fraction algorithms for interval exchange maps: an introduction" preprint (2004), to appear in the proceedings of conference Frontiers in Number Theory, Physics and Geometry, Les Houches, 9 - 21 March 2003.

[Z1] A. Zorich "Finite Gauss measure on the space of interval exchange transformations. Lyapunov exponents" Annales de l'Institut Fourier Tome 46 fasc. 2 (1996) 325-370. MR:1393518 (97f:58081)

[Z2] A. Zorich "Deviation for interval exchange transformations" Ergod. Th. Dyn. Sys. 17 (1997), 1477-1499. MR.1488330 (99e:58124)

[Z3] A. Zorich "On Hyperplane Sections of Periodic Surfaces" Amer. Math. Soc. Translations 179 (1997), 173-189. MR:1437163 (98h:58148) 
[Z4] A. Zorich "How Do the Leaves of a Closed 1-form Wind Around a Surface?" in Pseudoperiodic Topology, V. Arnold, M. Kontsevich and A. Zorich editors, Amer. Math. Soc. Translations 197 (1999) 135-178. MR.1733872 (2001c:57019)

Scuola Normale Superiore, Piazza dei Cavalieri 7, 56126 Pisa, Italy

Service de Physique Théorique, Cea/Saclay, 91191 Gif-Sur-Yvette, France

Collège de France, 3, Rue d'Ulm, 75005 Paris, France 